\title{
CHARACTERIZING TRANSITION TEMPERATURE GAS IN THE GALACTIC CORONA
}

\author{
Bart P. Wakker ${ }^{1}$, Blair D. Savage ${ }^{1}$, Andrew J. Fox ${ }^{2,3,4}$, Robert A. Benjamin ${ }^{4}$, and Paul R. Shapiro ${ }^{6}$ \\ ${ }^{1}$ Department of Astronomy, University of Wisconsin, Madison, WI 53706, USA; wakker@astro.wisc.edu, savage@astro.wisc.edu \\ ${ }^{2}$ European Southern Observatory, Alonso de Córdova 3107, Casilla 19001, Vitacura, Santiago, Chile \\ ${ }^{3}$ Institute of Astronomy, University of Cambridge, Madingley Road, Cambridge CB3 OHA, UK \\ ${ }^{4}$ Space Telescope Science Institute, 3700 San Martin Drive, Baltimore MD 21218, USA; afox@stsci.edu \\ ${ }^{5}$ Department of Physics, University of Wisconsin-Whitewater, 800 West Main Street, Whitewater, WI 53190, USA; benjamir@uww.edu \\ ${ }^{6}$ University of Texas at Austin, Department of Astronomy, Austin, TX 78712, USA; shapiro@astro.as.utexas.edu \\ Received 2011 August 10; accepted 2012 January 27; published 2012 April 3
}

\begin{abstract}
We present a study of the properties of the transition temperature $\left(T \sim 10^{5} \mathrm{~K}\right)$ gas in the Milky Way corona, based on the measurements of O VI, N v, C IV, Si IV, and Fe III absorption lines seen in the far-ultraviolet spectra of 58 sight lines to extragalactic targets, obtained with the Far-Ultraviolet Spectroscopic Explorer and the Space Telescope Imaging Spectrograph. In many sight lines the Galactic absorption profiles show multiple components, which are analyzed separately. We find that the highly ionized atoms are distributed irregularly in a layer with a scale height of about $3 \mathrm{kpc}$, which rotates along with the gas in the disk, without an obvious gradient in the rotation velocity away from the Galactic plane. Within this layer the gas has randomly oriented velocities with a dispersion of 40-60 $\mathrm{km} \mathrm{s}^{-1}$. On average the integrated column densities are $\log N(\mathrm{O} \mathrm{vI})=14.3, \log N(\mathrm{~N} \mathrm{v})=13.5$, $\log N(\mathrm{C}$ IV $)=14.2, \log N(\mathrm{Si}$ IV $)=13.6$, and $\log N(\mathrm{Fe}$ III $)=14.2$, with a dispersion of just 0.2 dex in each case. In sight lines around the Galactic center and Galactic north pole, all column densities are enhanced by a factor $\sim 2$, while at intermediate latitudes in the southern sky there is a deficit in $N(\mathrm{O} \mathrm{vI})$ of about a factor of two, but no deficit for the other ions. We compare the column densities and ionic ratios to a series of theoretical predictions: collisional ionization equilibrium, shock ionization, conductive interfaces, turbulent mixing, thick disk supernovae, static non-equilibrium ionization (NIE) radiative cooling, and an NIE radiative cooling model in which the gas flows through the cooling zone. None of these models can fully reproduce the data, but it is clear that NIE radiative cooling is important in generating the transition temperature gas.
\end{abstract}

Key words: Galaxy: halo - ISM: clouds - ISM: general - ultraviolet: ISM

Online-only material: color figure, figure set, machine-readable table

\section{INTRODUCTION}

Understanding the processes in Milky Way disk, thick disk, and halo gas that generate gas at temperatures $T>10^{5} \mathrm{~K}$ is a crucial element in learning about the lifecycle of gas in galaxies. Such gas can be produced by supernovae, winds, and infall, and its presence traces the circumstances of galactic evolution. Spitzer (1956) originally proposed the existence of a "hot interstellar Galactic corona," with $T \sim 10^{6} \mathrm{~K}$, to account for the presence of neutral clouds about $1 \mathrm{kpc}$ above the Galactic plane (Münch 1952; Münch \& Zirin 1961), the idea being that these clouds could only persist if they were confined by the pressure of an external medium. After the detection of the diffuse galactic soft X-ray background (Bunner et al. 1973; Williamson et al. 1974) and of O VI absorption (Rogerson et al. 1973; York 1974; Jenkins \& Meloy 1974), Shapiro \& Field (1976) proposed that this hot gas would produce a "Galactic Fountain"- a process in which the interstellar medium (ISM) is heated by supernova explosions, expands away from the Galactic plane, tries to settle hydrostatically, but as it cools clouds with $T \sim 10^{4} \mathrm{~K}$ rain back on the disk. This is a natural way to produce moving clouds of cooler gas embedded in a hot medium. The precise predictions of the process depend on many factors, most importantly the ratio of free-fall time to cooling time (Bregman 1980; Kahn 1981; Houck \& Bregman 1990). Other ways to produce hot coronal gas have also been proposed, e.g., a Galactic wind originating in the center (see Everett et al. 2008 and references therein) or heat generated by accreting clouds (considered by Henley et al. 2010).
In his original paper, Spitzer (1956) pointed out that the $\left(1 s^{2} 2 s\right)^{2} S_{1 / 2} \rightarrow\left(1 s^{2} 2 p\right)^{2} P_{1 / 2,3 / 2}$ electronic transitions of the lithium-like ions $\mathrm{O}^{+5}, \mathrm{~N}^{+4}$, and $\mathrm{C}^{+3}$ (and the similar $\mathrm{Si}$ ion $\mathrm{Si}^{+3}$ ) produce resonance doublet absorption lines in the UV (at $\lambda \sim 1030,1240,1550$, and 1400 A, respectively). These ions have ionization potentials for production of 113.9, 77.5, 47.9, and $33.5 \mathrm{eV}$ and trace gas in the $(1-3) \times 10^{5} \mathrm{~K}$ temperature range, which originates in processes that convert $10^{6} \mathrm{~K}$ into $10^{4} \mathrm{~K}$ gas and vice versa. At the temperatures sampled by these ions, the ISM is unstable-left to itself it will rapidly cool to below $10^{4} \mathrm{~K}$. The presence of these ions therefore implies the existence of $10^{6} \mathrm{~K}$ or hotter gas that has cooled down or of a process that is heating cooler gas.

Here two notes about terminology are in order. First, Spitzer used "corona" for the region containing hot gas out to a few kpc from the Galactic plane. "Halo" has often been used instead, referring to the region of space outside the Galactic disk. As the scale heights of $\mathrm{O} v \mathrm{VI}, \mathrm{N} v$, and $\mathrm{C}$ IV are found to be only $\sim 3 \mathrm{kpc}$, Savage et al. (2003) refer to the region where the $10^{5} \mathrm{~K}$ gas occurs as a "thick disk," as opposed to a much more extended $(>50 \mathrm{kpc})$ corona that is implied by the detection of $\mathrm{O}$ VI absorption associated with the Magellanic Stream (Sembach et al. 2003). We will follow Savage et al. (2003) in using this terminology. Second, the term "hot gas" has often been used to describe gas with $T>10^{5} \mathrm{~K}$, using "warm gas" if the temperature is $\sim 5000 \mathrm{~K}$ to a few $10^{4} \mathrm{~K}$. However, since gas at temperatures $\sim 10^{5} \mathrm{~K}$ has very different properties than gas at $T \sim 10^{6} \mathrm{~K}$ (not the least of which is that it cools rapidly), we will instead follow the convention of Savage \& Wakker (2009), 
who proposed to instead use the term "transition temperature gas" for this quickly evolving phase, reserving "hot gas" for the X-ray-emitting phase with $T>10^{6} \mathrm{~K}$.

The desire to detect the highly ionized interstellar atoms was one of Spitzer's main motivators to push for the creation of a UV satellite in space, resulting in the launch of the Copernicus satellite in 1972. Other UV spectroscopic instruments followed, including the International Ultraviolet Explorer (IUE), the Goddard High Resolution Spectrograph (GHRS), the Space Telescope Imaging Spectrograph (STIS), the Far-Ultraviolet Spectroscopic Explorer (FUSE), and the Cosmic Origins Spectrograph (COS). These instruments can detect the highly ionized ions in the Milky Way disk and corona in absorption against both stars and extragalactic targets. Many such studies have been done: York (1974, 1977), Jenkins \& Meloy (1974), Jenkins (1978a, 1978b), Cowie et al. (1979, 1981), Savage \& de Boer (1979, 1981), Savage \& Massa (1987), Sembach et al. (1990, 1991, 1994a, 1994b, 1995, 1999, 2001), Savage et al. (1990, 1995, 1997a, 1997b, 2001a, 2001b, 2003, 2005), Sembach \& Savage (1992, 1994), Tripp et al. (1993), Savage \& Sembach (1994), Sembach (1994), Jenkins et al. (1998, 2000), Oegerle et al. (2000, 2005), Sterling et al. (2002), Howk et al. (2002, 2003), Fox et al. (2003), Zsargó et al. (2003), Wakker et al. (2003), Knauth et al. (2003), Lehner et al. (2003), Indebetouw \& Shull (2004b), Ganguly et al. (2005), Savage \& Lehner (2006), Keeney et al. (2006), Bowen et al. (2008), Savage \& Wakker (2009), and Lehner et al. (2011). These studies show that in the disk the average density of the ionized hydrogen plasma in which the highly ionized species occur is about $3 \times 10^{-4} \mathrm{~cm}^{-3}$, while the scale height of the ions is $2.6 \pm 0.6 \mathrm{kpc}$ for $\mathrm{O}$ vi, $3.6_{-0.8}^{+1.0} \mathrm{kpc}$ for $\mathrm{C}$ IV , and $3.2_{-0.6}^{+1.0} \mathrm{kpc}$ for Si IV. The velocity spread of the gas is too low to explain the scale height as due to turbulent pressure. The distribution is highly irregular. Finally, the ionization conditions show that the gas is far out of equilibrium.

Starting in the early 1990s, the GHRS and STIS instruments on the Hubble Space Telescope (HST) have observed 85 extragalactic targets, usually for the purpose of studying intergalactic absorption in the sight lines. Toward each of these sight lines, absorption by Galactic N v, C IV, and/or Si IV is seen. Combined with the $\sim 150$ sight lines toward which $O$ vi can be measured using FUSE, this data set presents an opportunity to learn more about the properties of the transition temperature gas in the Milky Way, allowing us to address issues such as its kinematics relative to differential galactic rotation, its vertical velocity spread, the total amount, its distribution on the sky and relation to other structures, and the physical processes that produce it.

In this paper, we provide a table of extragalactic targets observed with HST spectrographs between 1992 and 2007, i.e., until the breakdown of the STIS spectrograph (Section 2). At the time this paper was finished (mid 2011), the COS spectrograph (launched in fall 2009) had already produced many new spectra covering N v, C IV, and Si IV absorption. However, as of 2011 September only a handful are public, and only two sight lines would be added to our sample of sight lines for which high signal-to-noise ratio $(\mathrm{S} / \mathrm{N})$ data are available for all four highly ionized species. Future studies will allow detailed studies of $\mathrm{N} \mathrm{v}$, $\mathrm{C}$ IV, and Si IV in a few hundred sight lines (although O vi will be missing for most of these new sight lines). For each of the targets observed by $H S T$ before 2007, we include FUSE observations if they are available. We then determine the absorption that we think is associated with the thick disk of the Galaxy (Section 3). In a large fraction of the sight lines, absorption is also visible at high velocities $\left(\left|v_{\mathrm{LSR}}\right|>100 \mathrm{~km} \mathrm{~s}^{-1}\right.$; see, e.g., Sembach et al. 2003; Fox et al. 2006). We do not include those components in this paper. The data also include absorption lines of ions with low-ionization potential (C I, C II, N I, O I, Si II, S II, Ar I, Fe II). We will not address these ions here. A few abundant ions with intermediate-ionization potentials (13.6-35 eV) also produce strong UV absorption lines (C III, N III, O III, Al III, Si III, S III, and Fe III). Of this set, the Galactic C III $\lambda 977.020$ and Si III $\lambda 1206.500$ absorption are always saturated, the Galactic O III and most of the N III lines are in the EUV $(\lambda<912 \AA)$ and thus hidden by the Galactic H I absorption, and N III $\lambda 989$ blends with a strong Si II line. Al III $\lambda 1854$ was observed by IUE toward many stars (see, e.g., Savage \& Wakker 2009), but lies outside the wavelength range of most existing HST spectra. However, S III $\lambda 1012.501$ and Fe III $\lambda 1122.524$ are visible in FUSE spectra (although the $\mathrm{S}$ III line is usually contaminated by Galactic $\mathrm{H}_{2}$ absorption). In this paper, we will include $\mathrm{S}$ III and Fe III in our analyses, allowing a comparison of the properties of the warm ionized medium with the more highly ionized transition temperature gas.

We list the measured column densities and other parameters of O vi, N v, C IV, Si IV, Fe III, and S III for the thick disk component of the high-ion absorption lines in Section 3. Section 4 presents a discussion of the properties of the absorption, such as their distribution across the sky, the kinematical relationships between the different ions, and the column density ratios. We address the physical processes that cause these ions to appear in Section 5, where we present a discussion of the predictions for ionic column density ratios implied by the various models that have been proposed. Compared to earlier discussions (Spitzer 1990; Savage et al. 1997a; Fox et al. 2004; Indebetouw \& Shull 2004a), we look more precisely at the numerical predictions for ionic ratios, and we consider several new models. Finally, we discuss what we have learned in Section 6.

\section{OBSERVATIONS}

\subsection{Sources of Data}

In Table 1, we give observational information for the high-resolution $(R>10,000)$ GHRS, STIS-G140M, or STIS-E140M spectra that were taken before 2007 and for which the $\mathrm{S} / \mathrm{N}$ per $20 \mathrm{~km} \mathrm{~s}^{-1}$ near either the $\mathrm{N} \mathrm{v}$ or $\mathrm{C}$ IV line is larger than 7 . This $\mathrm{S} / \mathrm{N}$ limit is used because at lower $\mathrm{S} / \mathrm{N}$ ratios the Galactic absorption lines become difficult to measure and the error in the ratios derived between ionic column densities is larger than a factor of two.

Of the set of observations, 9 targets were only observed using the GHRS, 18 were only observed using STIS-G140M, and 31 were observed using STIS-E140M. Of the 58 targets used in our analysis, 43 also have FUSE data with S/N near O vi greater than 7 . There are 55 targets having data with $\mathrm{S} / \mathrm{N}>7$ for $\mathrm{N} \mathrm{v}$, and 33 have such data covering C IV, while for 26 targets the $\mathrm{S} / \mathrm{N}$ ratio near all three lines is $>7$. These 26 form the main sample for the current study.

Figure 1 shows the distribution of the analyzed targets on the sky. The integrated Galactic H I column density is also shown, with a contour at an $\mathrm{H}$ I column density of $7 \times 10^{20} \mathrm{~cm}^{-2}$. This shows that the extragalactic UV-bright targets that can be observed with space spectrographs only sample gas at high Galactic latitudes, and that these targets are spread fairly evenly across the sky, with the exception of a concentration of targets in the "hole" near $l=120^{\circ}, b=45^{\circ}$, where the extinction is below average, allowing more targets to shine through the 
Table 1

Observational Data

\begin{tabular}{|c|c|c|c|c|c|c|c|c|c|c|c|}
\hline $\begin{array}{l}\text { Object } \\
\text { (1) }\end{array}$ & $\begin{array}{l}\text { Lon. } \\
\text { (deg) } \\
(2)\end{array}$ & $\begin{array}{l}\text { Lat. } \\
\text { (deg) } \\
(3)\end{array}$ & $\begin{array}{c}\text { Status }^{\mathrm{a}} \\
\text { (4) }\end{array}$ & $\begin{array}{c}\text { Instrument }^{b} \\
\text { (5) }\end{array}$ & $\begin{array}{c}\text { Resol. }^{\mathrm{c}} \\
\left(\mathrm{km} \mathrm{s}^{-1}\right) \\
(6)\end{array}$ & $\begin{array}{l}T_{\exp } \\
(\mathrm{ks}) \\
(7)\end{array}$ & $\begin{array}{l}\mathrm{S} / \mathrm{N} \\
\mathrm{O} \text { VI } \\
(8)\end{array}$ & $\begin{array}{l}\mathrm{S} / \mathrm{N} \\
\mathrm{N} \mathrm{v} \\
(9)\end{array}$ & $\begin{array}{l}\mathrm{S} / \mathrm{N} \\
\mathrm{C}_{\mathrm{IV}} \\
(10)\end{array}$ & $\begin{array}{l}\text { S/N } \\
\text { Si IV } \\
(11)\end{array}$ & $\begin{array}{l}\text { Obs. ID } \\
\text { (12) }\end{array}$ \\
\hline \multirow[t]{4}{*}{$3 \mathrm{C} 232$} & 194.17 & 52.32 & Skip & FUSE & 20 & 10.8 & 1 & & & & P10714 \\
\hline & & & & STIS-1222 & 30 & 11.2 & & 12 & & & O6700 \\
\hline & & & & STIS-1400 & 30 & 11.2 & & & & 11 & O6700 \\
\hline & & & & STIS-1567 & 30 & 22.3 & & & 13 & & O6700 \\
\hline \multirow[t]{2}{*}{ 3C249.1 } & 130.39 & 38.55 & Ratios & FUSE & 20 & 260.5 & 16 & & & & D11701 P10716 S60109 U10275 \\
\hline & & & & STIS-E140M & 8 & 137.6 & & 7 & 6 & 8 & O6E10 O6E12 O6E13 \\
\hline \multirow[t]{2}{*}{$3 \mathrm{C} 273.0$} & 289.95 & 64.36 & Ratios & FUSE & 20 & 43.3 & 27 & & & & P10135 \\
\hline & & & & STIS-E140M & 8 & 37.3 & & 28 & 21 & 43 & O5D30 \\
\hline 3C 351.0 & 90.08 & 36.38 & Ratios & STIS-E140M & 8 & 156.3 & & 9 & 9 & 9 & O5790 \\
\hline \multirow[t]{5}{*}{ ESO141-G55 } & 338.18 & -26.71 & Ratios & FUSE & 20 & 40.9 & 16 & & & & I90401 \\
\hline & & & & GHRS-1241 & 19 & 15.6 & & 16 & & & Z3E70 \\
\hline & & & & GHRS-1247 & 19 & 9.8 & & 16 & & & Z3I70 \\
\hline & & & & GHRS-1400 & 16 & 9.8 & & & & 13 & $\mathrm{Z} 3 \mathrm{I} 70$ \\
\hline & & & & GHRS-1538 & 14 & 11.1 & & & 12 & & Z3I70 \\
\hline ESO438-G09 & 277.55 & 29.36 & Skip & STIS-1222 & 30 & 11.1 & & 10 & & & O5EW0 \\
\hline \multirow[t]{5}{*}{ Fairall9 } & 295.07 & -57.83 & Meas. & FUSE & 20 & 34.8 & 6 & & & & P10106 \\
\hline & & & & GHRS-1238 & 19 & 14.4 & & 26 & & & Z3E70 \\
\hline & & & & GHRS-1250 & 18 & 8.1 & & 26 & & & $\mathrm{Z} 26 \mathrm{O} 0$ \\
\hline & & & & GHRS-1258 & 18 & 6.9 & & & & & Z3E70 \\
\hline & & & & GHRS-1539 & 14 & 8.1 & & & 6 & & $\mathrm{Z} 26 \mathrm{OO}$ \\
\hline \multirow[t]{2}{*}{$\mathrm{H} 1821+643$} & 94.00 & 27.42 & Skip & FUSE & 20 & 278.4 & 28 & & & & C09502 P10164 \\
\hline & & & & STIS-E140M & 8 & 101.9 & & 13 & 16 & 19 & O5E70 \\
\hline \multirow[t]{2}{*}{ HE0226-4110 } & 253.94 & -65.77 & Ratios & FUSE & 20 & 204.8 & 24 & & & & D02701 P10191 P20713 \\
\hline & & & & STIS-E140M & 8 & 87.5 & & 8 & 8 & 11 & O6E10 O6E11 \\
\hline HE0340-2703 & 222.68 & -52.12 & Meas. & STIS-1222 & 30 & 4.9 & & 11 & & & O8EI0 \\
\hline \multirow[t]{2}{*}{ HE1029-1401 } & 259.33 & 36.52 & Meas. & STIS-1222 & 30 & 4.1 & & 33 & & & O4EC0 \\
\hline & & & & STIS-1272 & 30 & 3.4 & & & & & O4EC0 \\
\hline \multirow[t]{2}{*}{ HE1228+0131 } & 291.26 & 63.66 & Ratios & FUSE & 20 & 4.0 & 5 & & & & P10190 \\
\hline & & & & STIS-E140M & 8 & 54.5 & & 5 & 6 & 7 & O56A0 \\
\hline \multirow[t]{2}{*}{ HS0624+6907 } & 145.71 & 23.35 & Ratios & FUSE & 20 & 132.3 & 11 & & & & P10710 S60112 U10210 \\
\hline & & & & STIS-E140M & 8 & 123.9 & & 7 & 9 & 14 & O6E10 O6E11 \\
\hline \multirow[t]{4}{*}{ HS1543+5921 } & 92.40 & 46.36 & Skip & FUSE & 20 & 8.5 & 2 & & & & P10805 \\
\hline & & & & STIS-1222 & 30 & 25.2 & & 13 & & & O8MR0 \\
\hline & & & & STIS-1272 & 30 & 54.0 & & & & & O8MR0 \\
\hline & & & & STIS-1321 & 30 & 49.5 & & & & & O8MR0 \\
\hline MCG+10-16-111 & 144.21 & 55.08 & Meas. & STIS-1222 & 30 & 19.5 & & 30 & & & O5EW0 \\
\hline MRC2251-178 & 46.20 & -61.33 & Meas. & FUSE & 20 & 50.2 & 12 & & & & P11110 \\
\hline & & & & STIS-1222 & 30 & 6.0 & & 27 & & & O4EC0 \\
\hline & & & & STIS-1272 & 30 & 4.6 & & & & & O4EC0 \\
\hline Mrk33 & 156.20 & 52.80 & Skip & FUSE & 20 & 23.7 & 6 & & & & C04805 \\
\hline & & & & STIS -1222 & 30 & 15.8 & & 9 & & & O5CA0 \\
\hline Mrk110 & 165.01 & 44.36 & Meas. & FUSE & 20 & 11.5 & 1 & & & & $\mathrm{P} 10713$ \\
\hline & & & & STIS-1222 & 30 & 4.5 & & 10 & & & O4N30 O4N35 \\
\hline Mrk205 & 125.45 & 41.67 & Ratios & FUSE & 20 & 231.9 & 17 & & & & D05401 Q10602 S60108 U10311 \\
\hline & & & & STIS-E140M & 8 & 124.0 & & 8 & 6 & 9 & $\mathrm{O} 62 \mathrm{Q} 0$ \\
\hline Mrk279 & 115.04 & 46.86 & Ratios & FUSE & 20 & 235.3 & 44 & & & & C09002 D15401 F32501 P10803 \\
\hline & & & & STIS-E140M & 8 & 107.4 & & 30 & 21 & 25 & O6JM0 O8K10 \\
\hline Mrk290 & 91.49 & 47.95 & Ratios & FUSE & 20 & 112.9 & 19 & & & & D07601 E08401 P10729 \\
\hline & & & & GHRS-1250 & 18 & 7.1 & & 25 & & & Z3KH0 \\
\hline Mrk335 & 108.76 & -41.42 & Ratios & FUSE & 20 & 99.2 & 28 & & & & P10102 \\
\hline & & & & STIS-E140M & 8 & 32.3 & & 11 & 5 & 7 & O8N50 \\
\hline Mrk421 & 179.83 & 65.03 & Meas. & FUSE & 20 & 82.0 & 30 & & & & P10129 Z01001 \\
\hline & & & & GHRS-1239 & 19 & 15.7 & & 24 & & & Z2IA0 \\
\hline Mrk478 & 59.24 & 65.03 & Meas. & FUSE & 20 & 14.0 & 8 & & & & P11109 \\
\hline & & & & STIS-1222 & 30 & 7.6 & & 29 & & & $\mathrm{O} 4 \mathrm{EC} 1$ \\
\hline & & & & STIS-1272 & 30 & 6.3 & & & & & O4EC1 \\
\hline Mrk501 & 63.60 & 38.86 & Meas. & FUSE & 20 & 30.2 & 9 & & & & C08101 P10733 \\
\hline & & & & GHRS-1239 & 19 & 31.3 & & 11 & & & $\mathrm{Z} 1 \mathrm{~A} 65$ \\
\hline Mrk509 & 35.97 & -29.86 & Ratios & FUSE & 20 & 90.4 & 30 & & & & P10806 X01701 \\
\hline & & & & STIS-E140M & 8 & 15.7 & & 12 & 8 & 9 & O6AP0 \\
\hline Mrk771 & 269.44 & 81.74 & Meas. & FUSE & 20 & 6.3 & 5 & & & & P10723 \\
\hline & & & & STIS-1222 & 30 & 7.8 & & 24 & & & O4EC0 O4N30 \\
\hline & & & & STIS-1272 & 30 & 5.2 & & & & & $\mathrm{O} 4 \mathrm{EC} 0$ \\
\hline Mrk817 & 100.30 & 53.48 & Meas. & FUSE & 20 & 186.8 & 43 & & & & P10804 \\
\hline
\end{tabular}


Table 1

(Continued)

\begin{tabular}{|c|c|c|c|c|c|c|c|c|c|c|c|}
\hline $\begin{array}{l}\text { Object } \\
\text { (1) }\end{array}$ & $\begin{array}{l}\text { Lon. } \\
\text { (deg) } \\
(2)\end{array}$ & $\begin{array}{l}\text { Lat. } \\
\text { (deg) } \\
(3)\end{array}$ & $\begin{array}{c}\text { Status }^{\mathrm{a}} \\
\text { (4) }\end{array}$ & $\begin{array}{c}\text { Instrument }^{\mathrm{b}} \\
\text { (5) }\end{array}$ & $\begin{array}{c}\text { Resol. }^{\mathrm{c}} \\
\left(\mathrm{km} \mathrm{s}^{-1}\right) \\
(6)\end{array}$ & $\begin{array}{c}T_{\exp } \\
(\mathrm{ks}) \\
(7)\end{array}$ & $\begin{array}{l}\mathrm{S} / \mathrm{N} \\
\text { O VI } \\
(8)\end{array}$ & $\begin{array}{l}\mathrm{S} / \mathrm{N} \\
\mathrm{N} \mathrm{v} \\
(9)\end{array}$ & $\begin{array}{l}\text { S/N } \\
\text { C IV } \\
(10)\end{array}$ & $\begin{array}{l}\text { S/N } \\
\text { Si IV } \\
(11)\end{array}$ & $\begin{array}{l}\text { Obs. ID } \\
\text { (12) }\end{array}$ \\
\hline & & & & GHRS-1241 & 19 & 26.8 & & 40 & & & Z3E70 \\
\hline \multirow[t]{2}{*}{ Mrk876 } & 98.27 & 40.38 & Ratios & FUSE & 20 & 139.4 & 33 & & & & D02802 P10731 U10366 \\
\hline & & & & STIS-E140M & 6 & 58.4 & & 11 & 8 & 16 & O8NN0 \\
\hline \multirow[t]{3}{*}{ Mrk926 } & 64.09 & -58.76 & Skip & FUSE & 20 & 11.2 & 3 & & & & P10740 \\
\hline & & & & STIS-1222 & 30 & 3.9 & & 8 & & & O4EC1 \\
\hline & & & & STIS-1272 & 30 & 3.7 & & & & & O4EC1 \\
\hline \multirow[t]{4}{*}{ Mrk1044 } & 179.69 & -60.48 & Skip & FUSE & 20 & 12.6 & 6 & & & & D04101 \\
\hline & & & & STIS-1222 & 30 & 2.4 & & 29 & & & O8K40 \\
\hline & & & & STIS-1272 & 30 & 4.8 & & & & & O8K40 \\
\hline & & & & STIS-1567 & 30 & 2.4 & & & 12 & & O8K40 \\
\hline \multirow{2}{*}{ Mrk1095 } & 201.69 & -21.13 & Meas. & FUSE & 20 & 56.0 & 10 & & & & P10112 \\
\hline & & & & GHRS-1241 & 19 & 21.0 & & 20 & & & Z3E70 \\
\hline \multirow[t]{2}{*}{ Mrk1383 } & 349.22 & 55.12 & Ratios & FUSE & 20 & 63.1 & 23 & & & & P10148 P26701 \\
\hline & & & & STIS-E140M & 8 & 38.3 & & 9 & 8 & 8 & O8PG0 \\
\hline \multirow[t]{2}{*}{ Mrk1502 } & 123.75 & -50.18 & Meas. & FUSE & 20 & 39.1 & 7 & & & & P11101 \\
\hline & & & & GHRS-1239 & 19 & 49.5 & & 10 & & & Z1A60 Z2IA0 \\
\hline \multirow[t]{3}{*}{ Mrk1513 } & 63.67 & -29.07 & Meas. & FUSE & 20 & 64.3 & 15 & & & & P10183 \\
\hline & & & & STIS-1222 & 30 & 7.3 & & 23 & & & O4EC1 \\
\hline & & & & STIS-1272 & 30 & 6.2 & & & & & O4EC1 \\
\hline \multirow{3}{*}{ NGC 985} & 180.84 & -59.49 & Meas. & FUSE & 20 & 44.3 & 10 & & & & P10109 \\
\hline & & & & STIS-1222 & 30 & 3.7 & & 25 & & & O4EC1 \\
\hline & & & & STIS-1272 & 30 & 3.8 & & & & & O4EC1 \\
\hline \multirow[t]{2}{*}{ NGC 1705} & 261.08 & -38.74 & Ratios & FUSE & 20 & 24.0 & 27 & & & & A04601 \\
\hline & & & & STIS-E140M & 8 & 34.2 & & 13 & 8 & 13 & O58N0 \\
\hline \multirow[t]{2}{*}{ NGC 3516} & 133.24 & 42.40 & Meas. & FUSE & 20 & 114.5 & 9 & & & & G91701 P11104 P21101 \\
\hline & & & & STIS-E140M & 8 & 13.7 & & 2 & 20 & 3 & O57B0 \\
\hline \multirow[t]{2}{*}{ NGC 3783} & 287.46 & 22.95 & Ratios & FUSE & 20 & 178.4 & 26 & & & & B10701 E03101 P10133 \\
\hline & & & & STIS-E140M & 8 & 187.4 & & 34 & 35 & 28 & O57B0 O63M0 O63M1 O63M5 \\
\hline NGC 4051 & 148.88 & 70.09 & Skip & FUSE & 20 & 86.6 & 16 & & & & B06202 C01901 \\
\hline & & & & STIS-E140M & 8 & 20.6 & & 7 & 8 & 6 & O5F00 \\
\hline NGC 4151 & 155.08 & 75.06 & Ratios & FUSE & 20 & 96.6 & 36 & & & & C09201 P11105 P21102 \\
\hline & & & & STIS-E140M & 8 & 55.4 & & 21 & 17 & 22 & O5780 O5KT0 O5KT5 O61L0 O6JB0 \\
\hline NGC 4593 & 297.48 & 57.40 & Skip & STIS-E140M & 8 & 22.0 & & 10 & 7 & 7 & O5L50 \\
\hline NGC 5548 & 31.96 & 70.50 & Ratios & FUSE & 20 & 56.1 & 9 & & & & D15501 P10146 \\
\hline & & & & STIS-E140M & 8 & 107.5 & & 24 & 11 & 10 & O6JD0 O6KW0 \\
\hline & & & & STIS-E140M & 8 & 4.8 & & 24 & 11 & 10 & O4LL0 \\
\hline NGC 7469 & 83.10 & -45.47 & Ratios & FUSE & 20 & 44.0 & 16 & & & & C09001 P10187 \\
\hline & & & & STIS-E140M & 8 & 71.6 & & 25 & 11 & 12 & O6BN0 O8N50 \\
\hline PG0804+761 & 138.28 & 31.03 & Meas. & FUSE & 20 & 170.9 & 31 & & & & P10119 S60110 \\
\hline & & & & STIS-1222 & 30 & 7.3 & & 20 & & & O4EC0 O4N30 \\
\hline & & & & STIS-1272 & 30 & 4.2 & & & & & O4EC0 \\
\hline PG0953+414 & 179.79 & 51.71 & Ratios & FUSE & 20 & 75.2 & 24 & & & & P10122 \\
\hline & & & & STIS-E140M & 8 & 49.0 & & 9 & 9 & 10 & $\mathrm{O} 4 \mathrm{X} 00$ \\
\hline PG1001+291 & 200.08 & 53.21 & Skip & FUSE & 20 & 11.4 & 5 & & & & P20731 \\
\hline & & & & STIS-E140M & 8 & 96.8 & & 7 & 5 & 8 & O6E10 O6E11 O6E12 \\
\hline PG1004+130 & 225.12 & 49.12 & Skip & FUSE & 20 & 83.4 & 8 & & & & A03501 \\
\hline & & & & STIS-1222 & 30 & 5.2 & & 11 & & & O5EW0 \\
\hline PG1049-005 & 252.28 & 49.88 & Meas. & STIS-1222 & 30 & 1.5 & & 6 & & & O4N30 \\
\hline PG1116+215 & 223.36 & 68.21 & Ratios & FUSE & 20 & 77.0 & 25 & & & & P10131 \\
\hline & & & & STIS-E140M & 8 & 53.1 & & 12 & 10 & 15 & O5A30 O5E70 \\
\hline PG1211+143 & 267.55 & 74.32 & Ratios & FUSE & 20 & 52.2 & 17 & & & & P10720 \\
\hline & & & & STIS-E140M & 8 & 110.0 & & 18 & 14 & 20 & O61Y0 \\
\hline PG1216+069 & 281.07 & 68.14 & Ratios & FUSE & 20 & 12.4 & 4 & & & & P10721 \\
\hline & & & & STIS-E140M & 8 & 139.6 & & 8 & 7 & 8 & O6E10 O6E13 \\
\hline PG1259+593 & 120.56 & 58.05 & Ratios & FUSE & 20 & 528.1 & 38 & & & & P10801 U10318 \\
\hline & & & & STIS-E140M & 8 & 191.5 & & 7 & 8 & 11 & O63G0 O63G1 \\
\hline PG1302-102 & 308.59 & 52.16 & Ratios & FUSE & 20 & 144.7 & 15 & & & & P10802 \\
\hline & & & & STIS-E140M & 8 & 44.2 & & 7 & 5 & 5 & O5BU0 O5BU6 \\
\hline PG1341+258 & 28.71 & 78.15 & Skip & STIS-1222 & 30 & 8.1 & & 15 & & & O5EW0 \\
\hline PG1351+640 & 111.89 & 52.02 & Meas. & FUSE & 20 & 119.2 & 17 & & & & P10725 S60107 \\
\hline & & & & STIS-1222 & 30 & 8.5 & & 10 & & & O4EC5 \\
\hline & & & & STIS-1272 & 30 & 6.2 & & & & & O4EC5 \\
\hline PG1444+407 & 69.90 & 62.72 & Ratios & FUSE & 20 & 10.1 & 4 & & & & P10727 \\
\hline & & & & STIS-E140M & 8 & 97.2 & & 8 & 8 & 8 & O6E10 \\
\hline
\end{tabular}


Table 1

(Continued)

\begin{tabular}{|c|c|c|c|c|c|c|c|c|c|c|c|}
\hline (1) & $\begin{array}{l}\text { Lon. } \\
\text { (deg) } \\
(2)\end{array}$ & $\begin{array}{l}\text { Lat. } \\
\text { (deg) } \\
(3)\end{array}$ & $\begin{array}{c}\text { Status }^{a} \\
(4)\end{array}$ & $\begin{array}{c}\text { Instrument }^{b} \\
\text { (5) }\end{array}$ & $\begin{array}{c}\text { Resol. }^{\mathrm{c}} \\
\left(\mathrm{km} \mathrm{s}^{-1}\right) \\
(6)\end{array}$ & $\begin{array}{l}T_{\text {exp }} \\
(\mathrm{ks}) \\
(7)\end{array}$ & $\begin{array}{l}\mathrm{S} / \mathrm{N} \\
\mathrm{O} \text { VI } \\
(8)\end{array}$ & $\begin{array}{l}\mathrm{S} / \mathrm{N} \\
\mathrm{N} \mathrm{v} \\
(9)\end{array}$ & $\begin{array}{l}\mathrm{S} / \mathrm{N} \\
\mathrm{C}_{\text {IV }} \\
(10)\end{array}$ & $\begin{array}{l}\text { S/N } \\
\text { Si IV } \\
(11)\end{array}$ & $\begin{array}{l}\text { Obs. ID } \\
\text { (12) }\end{array}$ \\
\hline PG1718+481 & 74.38 & 34.83 & Meas. & $\begin{array}{l}\text { FUSE } \\
\text { STIS-1567 }\end{array}$ & $\begin{array}{l}20 \\
30\end{array}$ & $\begin{array}{l}59.5 \\
11.3\end{array}$ & 2 & & 22 & & $\begin{array}{l}\text { E01001 P21001 } \\
\text { O4VA0 }\end{array}$ \\
\hline PHL1811 & 47.47 & -44.81 & Ratios & $\begin{array}{l}\text { FUSE } \\
\text { STIS-E140M }\end{array}$ & $\begin{array}{r}20 \\
8\end{array}$ & $\begin{array}{l}72.7 \\
67.8\end{array}$ & 17 & 12 & 9 & 18 & $\begin{array}{l}\text { P10810 P20711 } \\
\text { O8D90 }\end{array}$ \\
\hline PKS0312-77 & 293.44 & -37.55 & Meas. & STIS-E140M & 8 & 46.3 & & 3 & 3 & 3 & O65T0 O65T1 \\
\hline PKS2005-489 & 350.37 & -32.60 & Meas. & $\begin{array}{l}\text { FUSE } \\
\text { STIS-1222 } \\
\text { STIS-1272 }\end{array}$ & $\begin{array}{l}20 \\
30 \\
30\end{array}$ & $\begin{array}{r}48.6 \\
6.1 \\
5.5\end{array}$ & 17 & 67 & & & $\begin{array}{l}\text { C14903 P10738 } \\
\text { O4EC0 } \\
\text { O4EC0 }\end{array}$ \\
\hline PKS2155-304 & 17.73 & -52.25 & Ratios & $\begin{array}{l}\text { FUSE } \\
\text { STIS-E140M }\end{array}$ & $\begin{array}{r}20 \\
8\end{array}$ & $\begin{array}{r}119.8 \\
57.0\end{array}$ & 31 & 11 & 11 & 15 & $\begin{array}{l}\text { P10807 } \\
\text { O5BY0 }\end{array}$ \\
\hline RX J0100.4-5113 & 299.48 & -65.84 & Meas. & $\begin{array}{l}\text { FUSE } \\
\text { STIS-1222 } \\
\text { STIS-1272 }\end{array}$ & $\begin{array}{l}20 \\
30 \\
30\end{array}$ & $\begin{array}{r}22.6 \\
2.3 \\
1.2\end{array}$ & 9 & 8 & & & $\begin{array}{l}\text { D80603 E89702 } \\
\text { O8P80 } \\
\text { O8P80 }\end{array}$ \\
\hline Ton S180 & 139.00 & -85.07 & Meas. & $\begin{array}{l}\text { FUSE } \\
\text { STIS-1222 } \\
\text { STIS-1272 }\end{array}$ & $\begin{array}{l}20 \\
30 \\
30\end{array}$ & $\begin{array}{r}26.7 \\
4.1 \\
3.5\end{array}$ & 14 & 27 & & & $\begin{array}{l}\text { D02801 P10105 } \\
\text { O4EC0 } \\
\text { O4EC0 }\end{array}$ \\
\hline Ton S210 & 224.97 & -83.16 & Ratios & $\begin{array}{l}\text { FUSE } \\
\text { STIS-E140M }\end{array}$ & $\begin{array}{r}20 \\
8\end{array}$ & $\begin{array}{r}52.5 \\
5.1\end{array}$ & 20 & 4 & 5 & 7 & $\begin{array}{l}\text { P10703 } \\
\text { O6L00 }\end{array}$ \\
\hline UGC12163 & 92.14 & -25.34 & Ratios & $\begin{array}{l}\text { FUSE } \\
\text { STIS-E140M }\end{array}$ & $\begin{array}{r}20 \\
8\end{array}$ & $\begin{array}{l}49.9 \\
20.6\end{array}$ & 8 & 4 & 2 & 4 & $\begin{array}{l}\text { B06201 } \\
\text { O5IT0 }\end{array}$ \\
\hline VIIZw118 & 151.36 & 25.99 & Meas. & $\begin{array}{l}\text { FUSE } \\
\text { STIS-1222 }\end{array}$ & $\begin{array}{l}20 \\
30\end{array}$ & $\begin{array}{r}194.4 \\
9.5\end{array}$ & 18 & 16 & & & $\begin{array}{l}\text { P10116 S60113 U10215 } \\
\text { O4EC1 }\end{array}$ \\
\hline
\end{tabular}

Notes.

"Status of the analysis- "Ratios" means that this sight line is used to analyze ionic ratios; "Measured" means that only one or two of the four ions were measured; "Skip" implies that the $\mathrm{S} / \mathrm{N}$ of the data is sufficient, but the measurement is impossible or ambiguous.

${ }^{\mathrm{b}}$ FUSE or HST spectrograph, with in the case of GHRS data the central wavelength of the grating setting added; an added central wavelength for STIS data means that the G140M grating was used, otherwise the spectrum was obtained using the E140M echelle.

${ }^{c}$ Approximate size of resolution element.

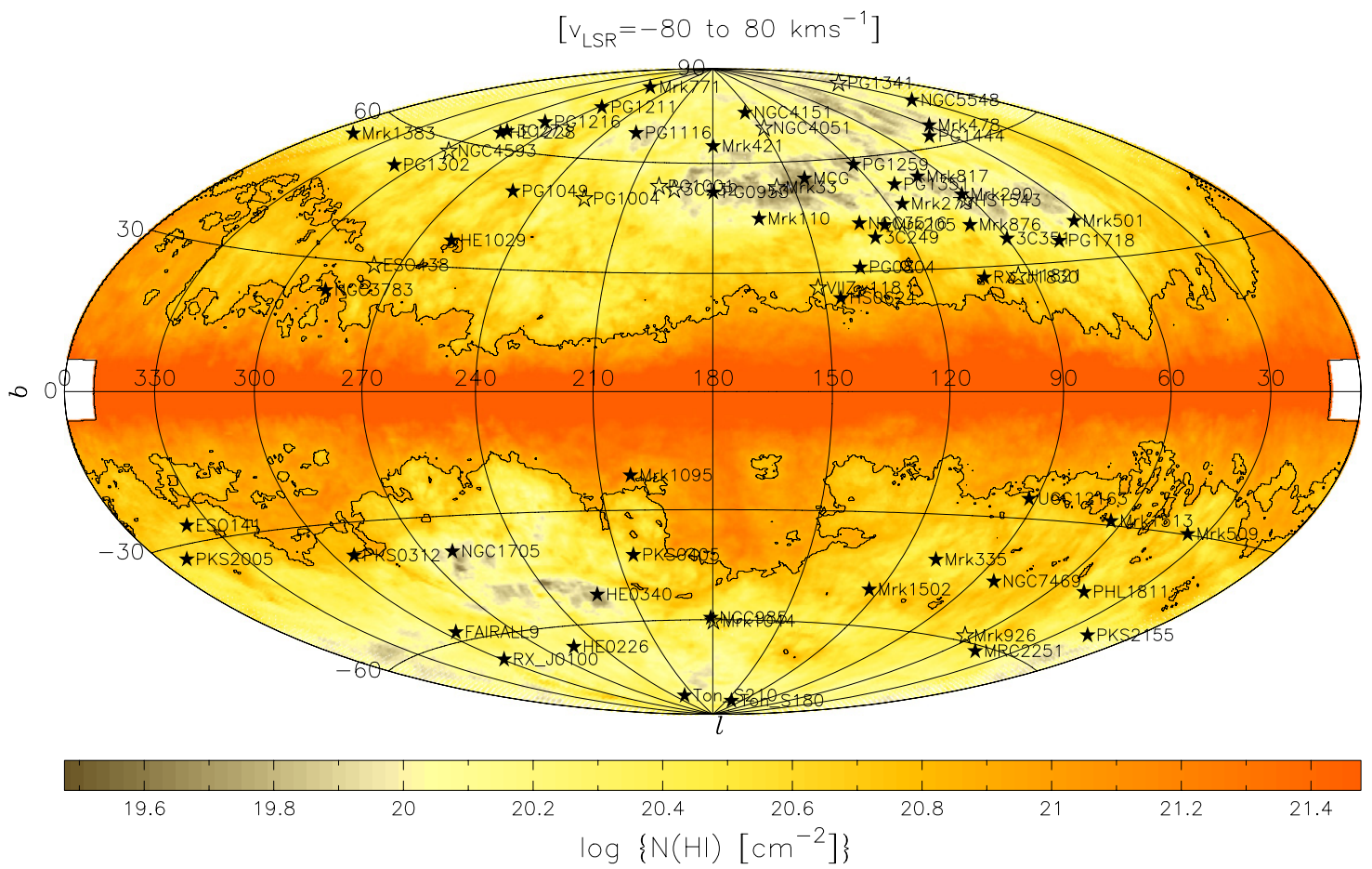

Figure 1. Distribution of targets with $\mathrm{N} v$ and/or C IV spectra, on top of a map of the logarithm of the column density of the $21 \mathrm{~cm} \mathrm{H} \mathrm{I} \mathrm{sky,} \mathrm{integrated} \mathrm{between}-80$ and $80 \mathrm{~km} \mathrm{~s}^{-1}$, with a contour overlaid for $N(\mathrm{HI})=7 \times 10^{20} \mathrm{~cm}^{-2}$. The data are shown in an Aitoff projection of Galactic coordinates centered on the anti-center direction. The H I data are from the Leiden-Argentina-Bonn (LAB) survey (Kalberla et al. 2005). 
Galactic dust. This is also the region of sky where the large highvelocity cloud (HVC) complex C can be found (Hulsbosch \& Wakker 1988), targets behind which have been disproportionally selected for observations with HST spectrographs.

Table 1 and Figure 1 include 12 targets for which the status column in the table has the value "Skip." This is the case when the spectra have a sufficiently high $\mathrm{S} / \mathrm{N}$ ratio, but there are problems with the measurements. For example, toward $\mathrm{H} 1821+643$ strong absorption is seen that is associated with the planetary nebula K1-16. For NGC 4151, both the STIS-E140M and FUSE spectrum have high S/N $(\sim 14)$, and Galactic absorption is clearly seen in all highly ionized species, but because of the low redshift of NGC 4151, it is blended with lines associated with the background galaxy, making the Galactic lines impossible to measure. For 3C 232 several STIS-G140M grating settings cover N v, C IV, and Si IV, but the absorptions in the individual lines of the CIV and Si IV doublet do not seem to match up. Toward PG 1004+130 the Galactic O VI appears to be broad, as do Si III $\lambda 1206.500$ and $\mathrm{C}_{\text {II }} \lambda$ 1036.337. However, a series of intrinsic $\mathrm{O}$ III lines probably contaminates the Galactic O vi. Eight other sight lines with high $\mathrm{S} / \mathrm{N}$ near $1238 \AA$ are excluded from the final sample (ESO 438-G09, HS 1543+5921, Mrk 33, Mrk 926, Mrk 1044, NGC 4593, PG 1001+291, and PG 1341+258). These have low $\mathrm{S} / \mathrm{N}$ or no data for $\mathrm{O}$ VI, and either the two lines of the $\mathrm{N} \mathrm{V}$ and/or C IV doublet do not match or there are intrinsic active galactic nucleus (AGN) lines contaminating the ISM lines. We still include these sight lines in Table 1, so that we can show their spectra in Figure 2, allowing the reader to check on the problems. However, they are not used in the analyses.

\subsection{Calibration}

For HST data, the calibrated fits files in the MAST archive were retrieved. This is the only step needed, except for observations with the STIS-G140M grating and central wavelength $1222 \AA$, where a 1 pixel shift seems necessary (see Wakker \& Savage 2009). That conclusion is based on 14 sight lines with good data and relatively simple ISM absorption lines. For these sight lines the $\mathrm{S}$ II $\lambda 1250.584,1253.811$ lines can be fit both in a G140M spectrum centered on $1222 \AA$ and in one centered on $1272 \AA$, and sometimes also in an E140M spectrum. To align the lines in the $1222 \AA$ centered spectrum with those in the other spectra, an average redward shift of $12 \mathrm{~km} \mathrm{~s}^{-1}$ is needed, which corresponds to 1 pixel.

Finally, for observations with the STIS-E140M echelle, the MAST fits files give the data for each of the 42 orders separately. These orders were combined into a single spectrum by interpolating the photon counts and errors onto a common grid, adding the photon counts and converting back to a flux.

The processing of the FUSE data was described in detail by Wakker et al. (2003) and Wakker (2006), and therefore only a summary is given here. First, the spectra were calibrated using version 2.1 or version 2.4 of the FUSE calibration pipeline. To correct for residual wavelength shifts, the central velocities of the Milky Way interstellar lines were determined for each detector segment $(\mathrm{LiF} 1 \mathrm{~A} / 1 \mathrm{~B} / 2 \mathrm{~A} / 2 \mathrm{~B}, \mathrm{SiC} 2 \mathrm{~A} / 2 \mathrm{~B})$ of each individual observation. The FUSE segments were then aligned with the local standard of rest (LSR) interstellar velocities implied by the STIS-E140M spectra, or, if no E140M data were available, with the LSR velocity of the strongest component in the $21 \mathrm{~cm} \mathrm{Hi} \mathrm{spectrum.} \mathrm{For} \mathrm{targets} \mathrm{with} \mathrm{an}$ STIS-E140M spectrum, the interstellar reference velocity was determined by fitting all Milky Way lines in that spectrum; the
STIS wavelength calibration is accurate to about $1 \mathrm{~km} \mathrm{~s}^{-1}$ (KimQuijano et al. 2007). For sight lines with $\mathrm{S} / \mathrm{N}>10$ near $1031 \AA$, the resulting shifts were given by Wakker (2006). Using these shifts, LiF1A and LiF2B data are added together to produce the final spectrum for each target. The final velocity scale references the velocities to the LSR.

\subsection{Presentation of Spectra}

We present the Galactic high-ionization absorption lines for six sight lines in Figure 2. These sight lines illustrate each of the items mentioned in the figure caption. The entire set of spectra is available in the electronic version of this paper. The plots include the available data for the O vI, N v, C IV, and Si IV doublets, as well as their apparent column density profiles. Note that we regridded the spectra to $10 \mathrm{~km} \mathrm{~s}^{-1}$ velocity bins for this figure. This does not affect the FUSE or STIS-G140M data, but does slightly degrade the STIS-E140M spectra (enhancing their $\mathrm{S} / \mathrm{N}$ ratio). The apparent column density is defined as

$$
N_{a}(v)=\frac{m_{e} c}{\pi e^{2}} f \lambda \ln \frac{C(v)}{F(v)},
$$

with $F(v)$ being the observed profile, $C(v)$ the fitted continuum, and $f$ being the oscillator strength. In addition, to allow the comparison of the kinematics of the high-ionization lines with those of lower ionization gas, we show a representative strong and weak line of an intermediate- and a low-ionization species, as described in the figure caption.

We also show profiles of the ratios between different ions. These are derived from the apparent column density profiles and their errors. We display the ionic ratio only in the range of velocities where it is larger than the error in this ratio, and only for sight lines and line combinations where both doublet lines of the various ions were detected at a significant level.

We show the actual spectra in Figure 2, i.e., they are not normalized relative to the continuum. However, for each absorption line we chose a vertical scale that places the continuum at about $80 \%$ of the maximum. For the apparent column density and ratio profiles, we use the same vertical scale for each ion throughout, to allow easy comparisons of the relative strength of the lines between different sight lines.

A sight line of note is NGC 4151 (spectra available in the online version of the journal). Here the $\mathrm{O}$ vi lines appear offset from all other absorption lines, which do line up with each other, even showing a component between -100 and $-60 \mathrm{~km} \mathrm{~s}^{-1}$ in C IV, Si III, and C II. There is no problem with the velocity calibration, as the Galactic $\mathrm{H}_{2}$ absorption lines near $\mathrm{O}$ VI $\lambda 1031.926$ are fitted perfectly well. The most likely explanation is that the $\mathrm{O}$ VI absorption between $v_{\mathrm{LSR}}=25$ and $60 \mathrm{~km} \mathrm{~s}^{-1}$ is related to the strong $\mathrm{P}$ Cygni profile produced by the outflowing gas in NGC 4151 itself. We include the measurements for most ions in this sight line, but we exclude the $\mathrm{O}$ vI lines.

We note that in all cases the profiles of the high-ionization species cover a similar range of velocities. In all but a few cases (PG 1116+215, PKS 2155-304; see online version), the shapes and velocity extent of the intermediate-ionization lines (i.e., $\mathrm{Si}$ III and Fe III) follow that of the high-ionization lines. On the other hand, the strong low-ionization absorption lines tend to be narrower than the high-ionization absorption lines. The weak low-ionization lines (usually exemplified by $\mathrm{S}_{\text {II }} \lambda 1250.584$ ) often show clear component structure, which is usually, but not always, reflected in the high-ionization absorption (contrast, e.g., ESO 141-G55, Mrk 205, PG 1302-102 with 3C 273.0, 


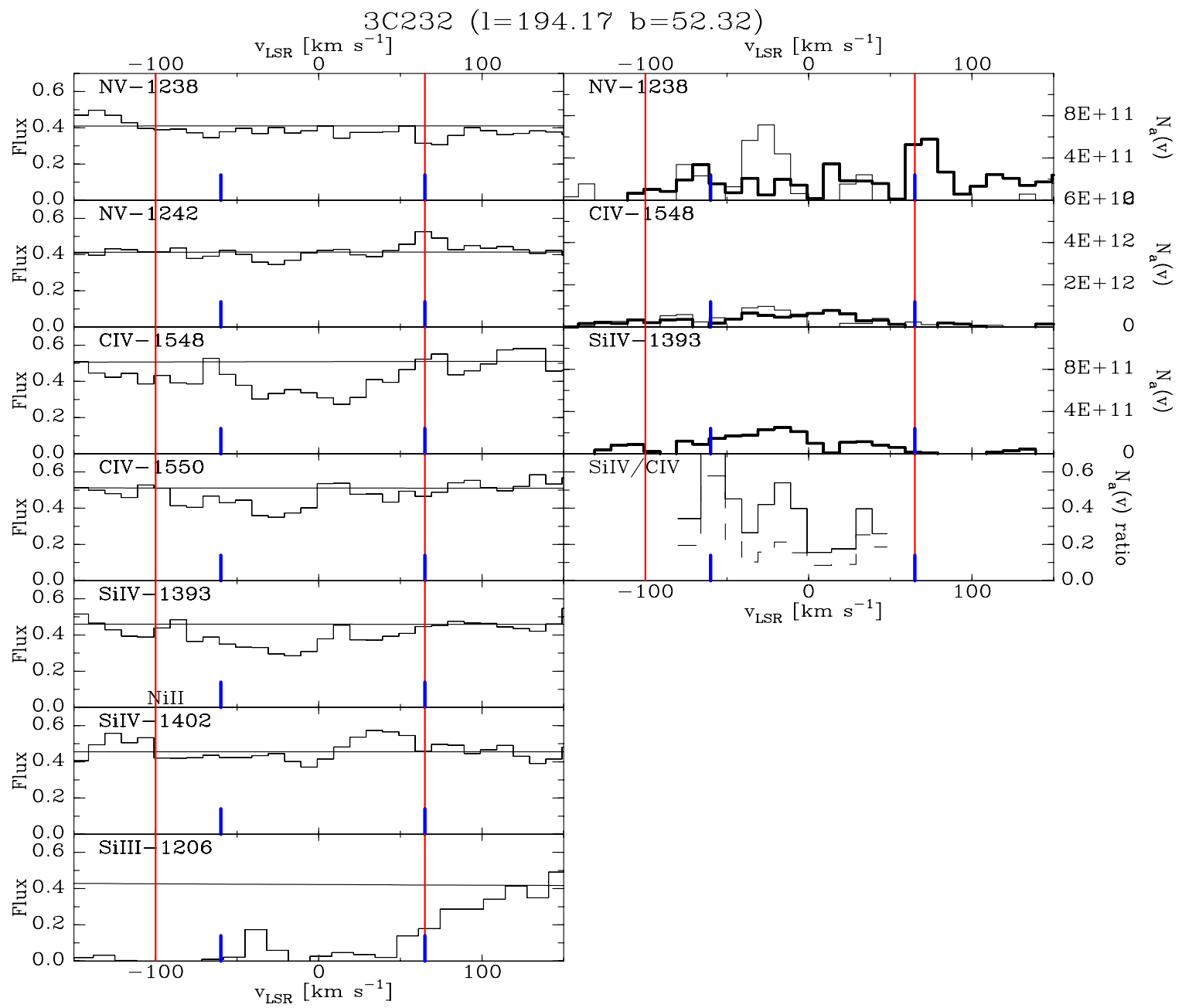

Figure 2. Panels show the available data for the four high-ionization lines, as well as for a strong and a weak intermediate-ionization and a strong and weak low-ionization line (when data are available). The panels on the left give the spectra for the high-ionization lines, as shown by the labels. The top set of panels on the right shows the apparent optical depth profiles for the high-ionization species, with the stronger absorption line shown as thick lines. No apparent optical depth profiles are shown for lines that were deemed unmeasurable, even though a panel is always shown if data were present. Below the optical depth profiles are panels showing the ratios between two ions, but only in spectral regions where the $\mathrm{S} / \mathrm{N}$ in the ratio is $>3$ in a $10 \mathrm{~km} \mathrm{~s}^{-1}$ velocity bin. Strong intermediate ionization is represented by $\mathrm{Si}$ III (or by $\mathrm{C}$ III when $\mathrm{Si}$ III is not available). Weak intermediate ionization is given by Fe III. The strong low-ionization line is either $\mathrm{C}$ II $\lambda 1334.532$ or $\mathrm{C}_{\text {II }} \lambda$ 1036.337, while the weak low-ionization line is S II $\lambda 1250.584$ or Ar I $\lambda 1048.220$. The intermediate- and low-ionization lines are always given in the bottom one or two panels. Line identifications are given at the top left of each panel. The continuum fit (including a model for $\mathrm{H}_{2}$ absorption) is also shown. In a few cases (e.g., for O VI $\lambda 1037.617$ toward Mrk 509), the continuum looks much higher than the data, because it was fit through pixels far outside the displayed range of velocities. All spectra were rebinned to $10 \mathrm{~km} \mathrm{~s}^{-1}$ pixels. Other absorption lines in each spectral window are identified by labels near the bottom of each panel. The short vertical blue lines show the range of velocities within $60 \mathrm{~km} \mathrm{~s}^{-1}$ of those consistent with standard Galactic differential rotation (see Section 4.2 for the justification of this number). The red vertical lines indicate the velocity limits of integration for the different Galactic components. Note that a figure is available for each sight line in the online version of the journal.

(A color version of this figure and the complete figure set (69 images) are available in the online journal.)

Mrk 509, PG 1116+215, and PKS 2005-489). Sometimes there is a narrow low-ionization component near $v \sim 0 \mathrm{~km} \mathrm{~s}^{-1}$, with no absorption at higher velocities, where there is strong high-ionization absorption (e.g., toward HE 0226-4110 and Mrk 509).

\section{MEASUREMENTS}

The Galactic high-ionization lines tend not to have simple Gaussian profiles, but rather they typically appear to be a mixture of several broad, blended components. Since the typical optical depth of the lines is on the order of one to two, and since we can only measure two lines for each ion, it is not advisable to estimate column densities by converting an equivalent width measurement into a column density or applying a curve-of- growth method. We also do not resort to fitting components to the line profiles as the $\mathrm{S} / \mathrm{N}$ is often low $(\sim 10-15)$, the profiles are usually asymmetric, and the component structure is not always clear. Profile fits would give formally correct results, and would allow us to calculate the total column density accurately, but the fitted central velocities and widths of the Gaussian components would more often than not be misleading, as a good fit would require several components, which usually cannot be defined unambiguously.

Because of these considerations, we instead integrated the apparent column density $\left(N_{a}(v)\right)$ profiles (Savage \& Sembach 1991). For resolved and unsaturated lines this represents the true column density distribution of the ion, broadened by the instrumental profile. If the $N_{a}(v)$ profiles of the two members 


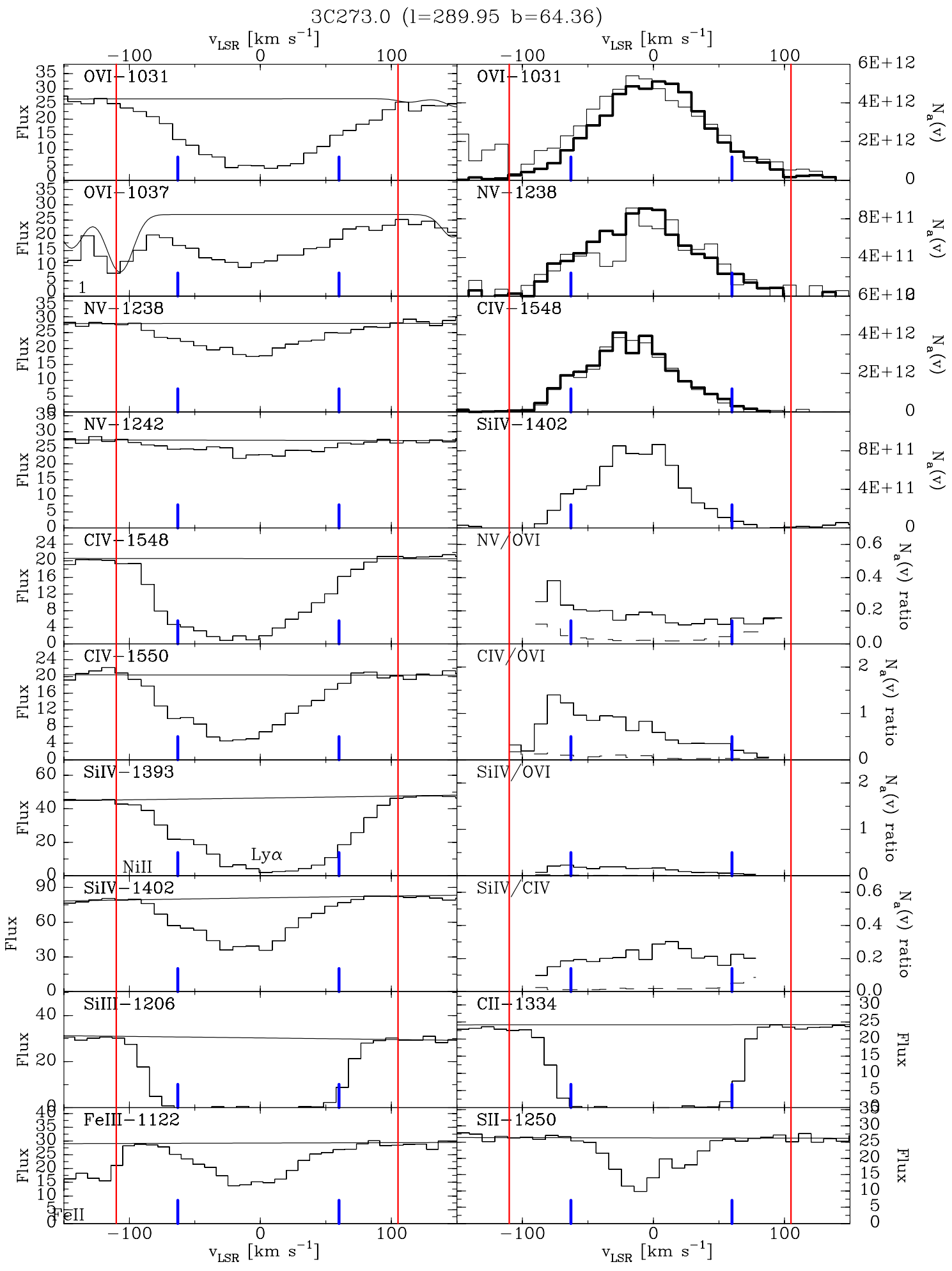

Figure 2. (Continued)

of each doublet match, then one can conclude that there is no unresolved saturation. In practice we find that this is a good assumption for the Galactic high-ionization lines, as the resulting column densities always match to within the errors (with one exception; see below).

To derive the column density profiles, we first fitted a local continuum to each of the absorption lines. To do this, we started by selecting line-free regions in a window about $\pm 1000 \mathrm{~km} \mathrm{~s}^{-1}$ wide around each line and then fitted a first-, second-, or third-order Legendre polynomial to these points, following the method of Sembach \& Savage (1992). In addition, a model for the molecular hydrogen absorption is applied, using the $\mathrm{H}_{2}$ column densities found by Wakker (2006). Any associated $\mathrm{H}_{2}$ optical depth is removed from the apparent optical depth profile. This is mostly important for the $\mathrm{O}$ VI $\lambda 1031.926$ line, while for $\mathrm{O}$ VI $\lambda 1037.617$ and $\mathrm{S}$ III $\lambda 1012.501$ the $\mathrm{H}_{2}$ contamination is often too large to be removed reliably. We only list the results for those two lines if the contamination is sufficiently minor. In 


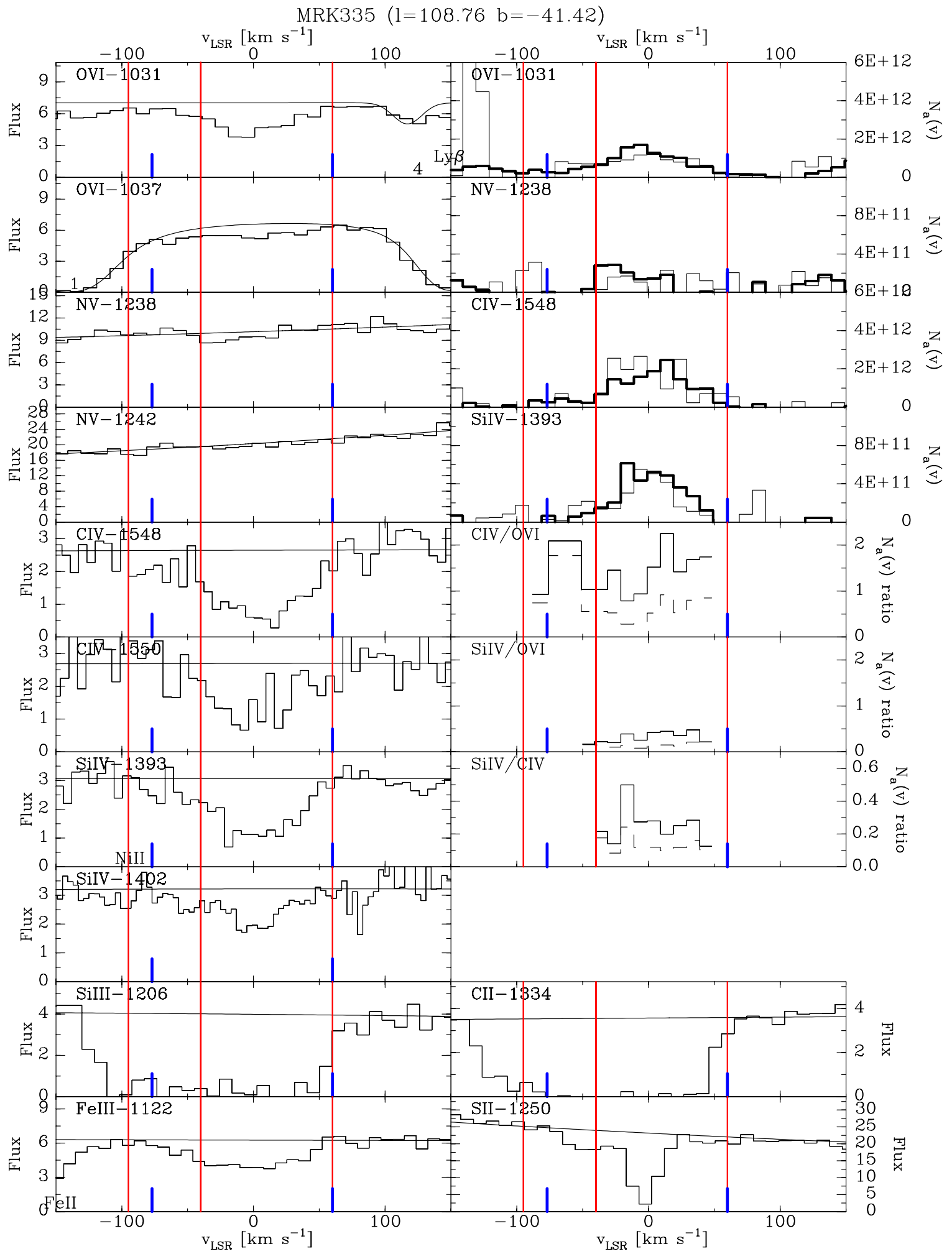

Figure 2. (Continued)

some sight lines we list the measurements of $\mathrm{H}_{2}$-decontaminated O vi lines, but do not use them for the analyses.

We visually determined the velocity extent of the absorption associated with the Milky Way thick disk. In many sight lines absorption occurs at velocities more negative than $-80 \mathrm{~km} \mathrm{~s}^{-1}$ or more positive than $80 \mathrm{~km} \mathrm{~s}^{-1}$. This high-velocity absorption was analyzed by Sembach et al. (2003). It is often associated with known $21 \mathrm{~cm}$ Hi HVCs, which trace the accretion of low-metallicity material and tidal streams (Wakker et al. 1999, 2007; Richter 2006). In this paper, we concentrate on the highionization absorption associated with the gas at velocities below about $80 \mathrm{~km} \mathrm{~s}^{-1}$, which originates in the disk and lower Galactic halo (i.e., the thick disk). An analysis of the high-velocity gas is deferred to the future. 


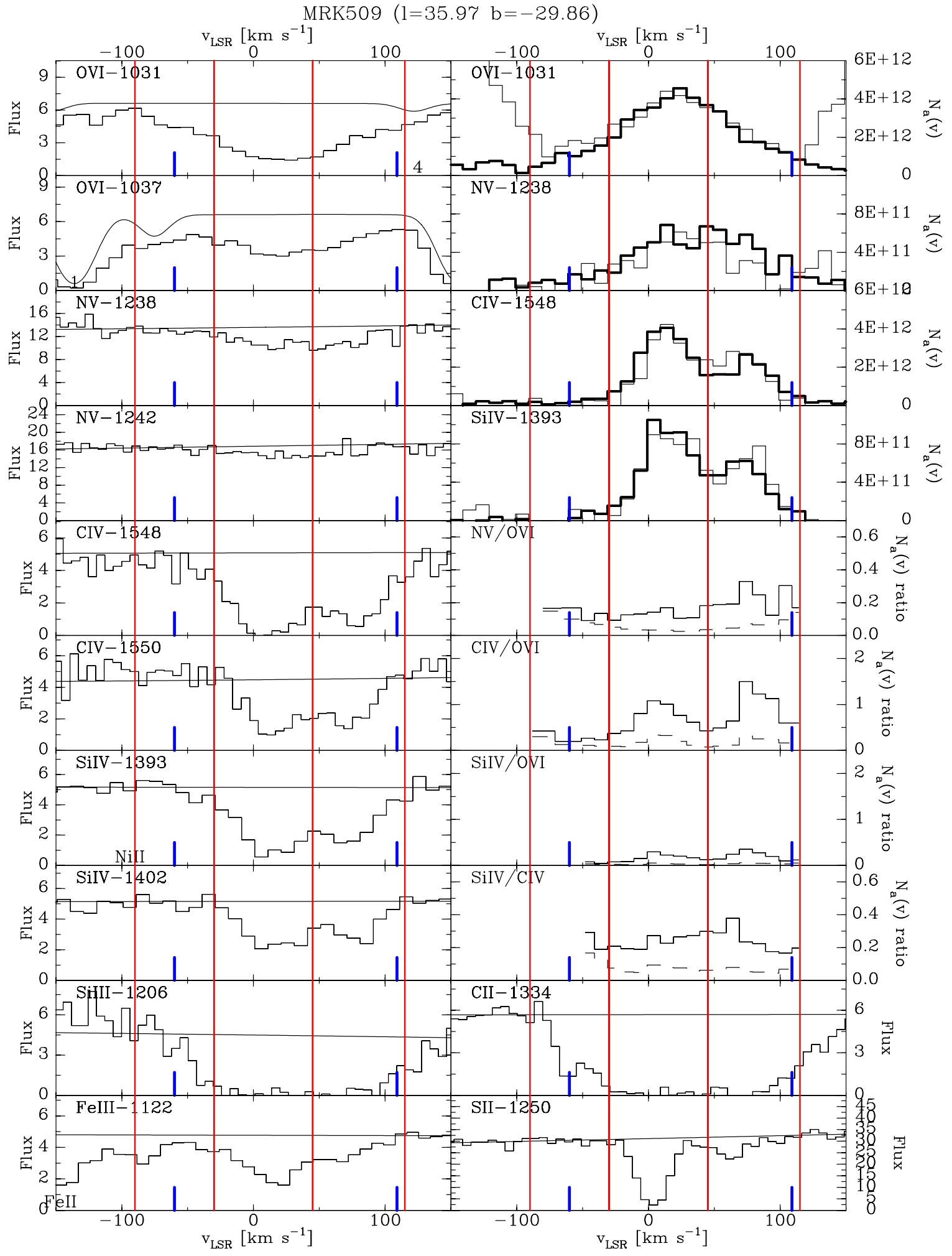

Figure 2. (Continued)

Next, we looked at the structure of all absorption lines toward each target and used the visual appearance of each profile and the comparison between the profiles for different ions (high-, intermediate-, and low-ionization species) to decide whether one can discern individual components and in which velocity ranges these occur. The structure in the low-ionization lines is taken into consideration for the determinations, but it does not drive the resulting interpretation for the high-ionization line component structure. We also noted cases where the line of the highly ionized species is blended with other galactic or intergalactic absorption lines. 


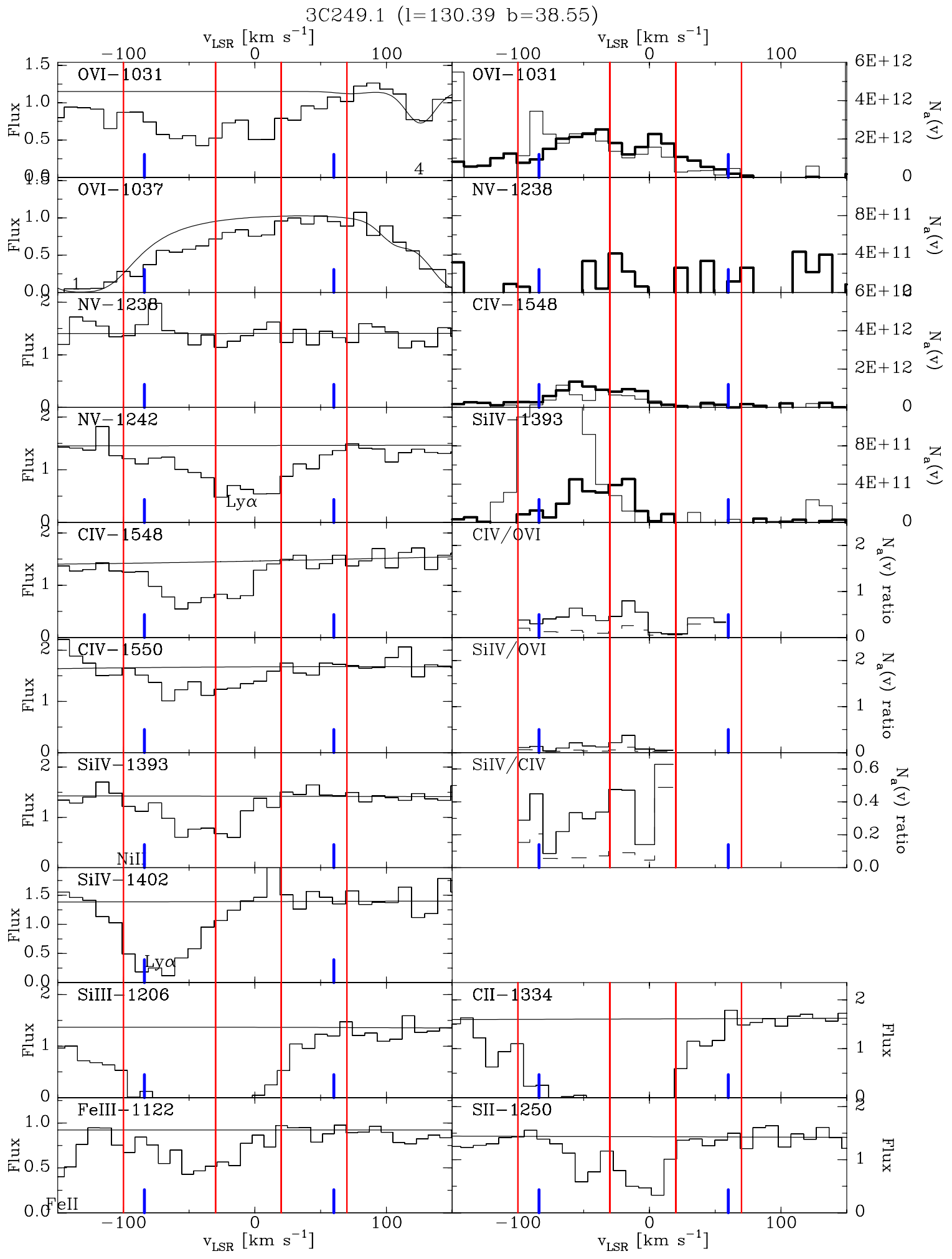

Figure 2. (Continued)

We then integrated the $N_{a}(v)$ profile, both over the full velocity range over which we think absorption originates in the thick disk, and over the different selected velocity ranges for each individual component. For each $N_{a}(v)$ integration, we determined a statistical and a systematic error, following the methods described by Wakker et al. (2003). The statistical error combines the Poisson noise, detector background, and continuum placement, while the systematic error takes into account the placement of integration limits and fixed-pattern noise. We flagged the cases where we judged it possible to derive reasonable values for line ratios.

We also calculated a central velocity $\left(v_{0}\right)$ and FWHM ( $W$, $W=\sqrt{4 \ln 2} b$, where $b$ is the Doppler parameter) line width from the first and second moments of the $N_{a}(v)$ profile. For the analyses we also correct the derived FWHMs for instrumental 


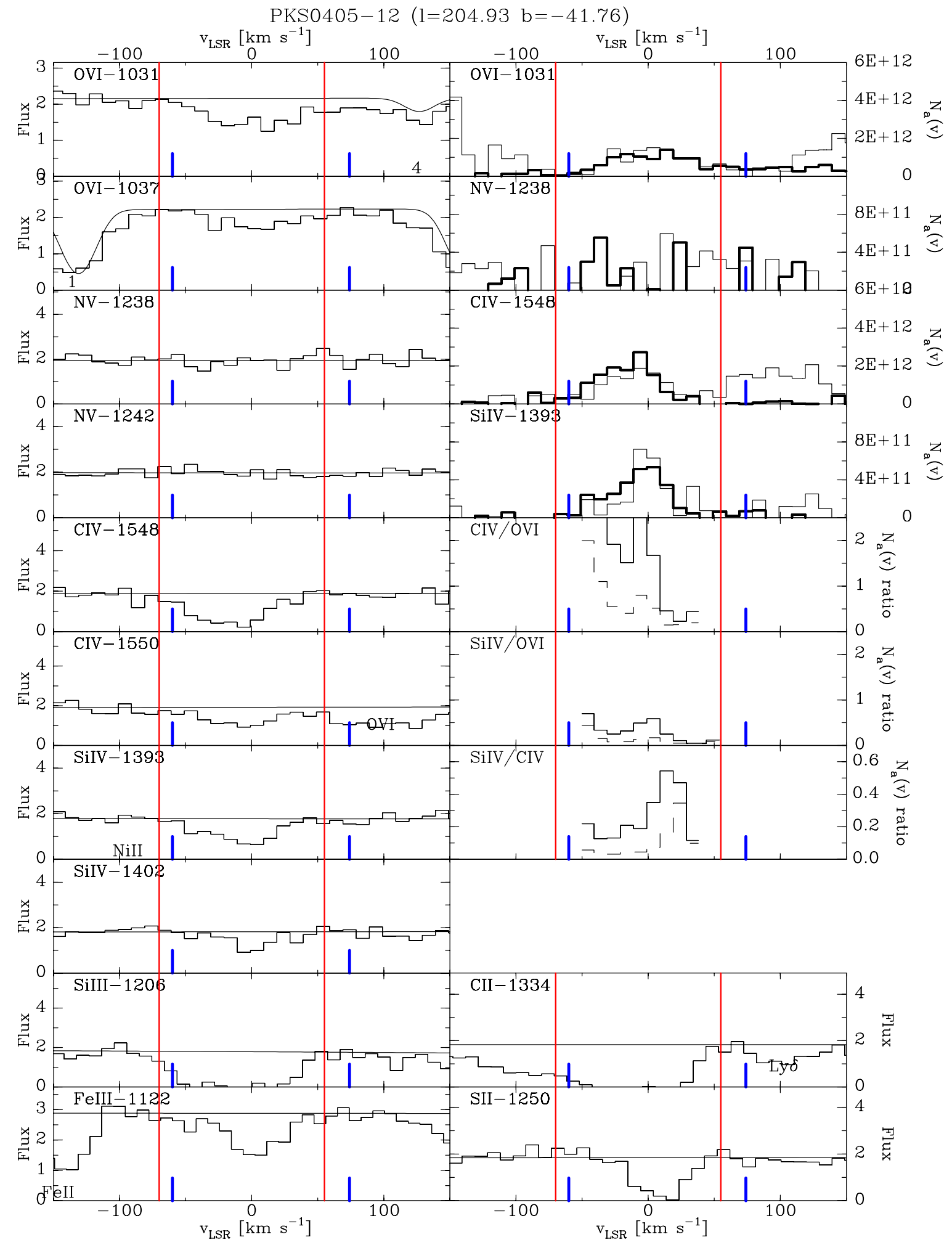

Figure 2. (Continued)

broadening (see notes to Table 2 for details):

$$
\begin{aligned}
N & =\int N_{a}(v) d v \\
v_{0} & =\left[\int v N_{a}(v) d v\right] /\left[\int N_{a}(v) d v\right], \\
W & =\sqrt{(4 \ln 2)}\left(\left[\int\left(v-v_{0}\right)^{2} N_{a}(v) d v\right] /\left[\int N_{a}(v) d v\right]\right)^{2} .
\end{aligned}
$$

We applied our best judgments to determine whether the resulting values are likely to represent a real width, as opposed to just being set by the center and width of the integration range. Only in about half of the sight lines were the individual components separated clearly enough to derive individual velocities and widths. However, for the full thick disk integration we can derive a column density weighted average velocity and width in almost all cases. 
Table 2

Measurements ${ }^{\mathrm{a}}$

\begin{tabular}{|c|c|c|c|c|c|c|c|c|c|c|}
\hline \multirow{2}{*}{$\begin{array}{l}\text { Object } \\
\text { (1) } \\
\end{array}$} & \multirow{2}{*}{$\begin{array}{l}\text { Vel. Range } \\
\left(\mathrm{km} \mathrm{s}^{-1}\right) \\
(2)\end{array}$} & \multirow{2}{*}{$\begin{array}{l}\text { Ion } \\
\text { (3) }\end{array}$} & \multicolumn{3}{|c|}{ Strong Line } & \multicolumn{3}{|c|}{ Weak Line } & \multicolumn{2}{|c|}{ Ratios } \\
\hline & & & $\begin{array}{c}\log N \\
\left(\mathrm{~cm}^{-2}\right) \\
(4)\end{array}$ & $\begin{array}{c}\langle v\rangle \\
\left(\mathrm{km} \mathrm{s}^{-1}\right) \\
(5)\end{array}$ & $\begin{array}{c}\text { FWHM } \\
\left(\mathrm{km} \mathrm{s}^{-1}\right) \\
(6)\end{array}$ & $\begin{array}{c}\log N \\
\left(\mathrm{~cm}^{-2}\right) \\
(7)\end{array}$ & $\begin{array}{c}\langle v\rangle \\
\left(\mathrm{km} \mathrm{s}^{-1}\right) \\
(8)\end{array}$ & $\begin{array}{c}\text { FWHM } \\
\left(\mathrm{km} \mathrm{s}^{-1}\right) \\
(9)\end{array}$ & $\begin{array}{l}\text { Ions } \\
\text { (10) }\end{array}$ & $\begin{array}{c}\log \text { Ratio } \\
\text { (11) }\end{array}$ \\
\hline \multirow[t]{23}{*}{ 3C249.1 } & $-100: 70$ & O VI & $14.38 \pm 0.03 \pm 0.01$ & & & {$[14.36 \pm 0.05 \pm 0.17]$} & & & & \\
\hline & & $\mathrm{Nv}$ & $<13.32$ & & & {$[\mathrm{Ly} \alpha]$} & & & $\mathrm{Nv} / \mathrm{O} \mathrm{vI}$ & $<-1.06$ \\
\hline & & CIV & $13.86 \pm 0.04 \pm 0.01$ & $-38 \pm 2$ & $68 \pm 7$ & $13.85 \pm 0.08 \pm 0.01$ & $-35 \pm 2$ & $75 \pm 7$ & $\mathrm{C}_{\mathrm{IV}} / \mathrm{O}$ VI & $-0.52 \pm 0.09$ \\
\hline & & Si IV & $13.39 \pm 0.03 \pm 0.01$ & $-40 \pm 1$ & $58 \pm 6$ & {$[\operatorname{Ly} \alpha]$} & & & Si IV/C IV & $-0.47 \pm 0.09$ \\
\hline & & Fe III & $14.27 \pm 0.05 \pm 0.01$ & $-39 \pm 3$ & $72 \pm 7$ & & & & $\mathrm{Fe}$ III/C IV & $0.41 \pm 0.10$ \\
\hline & $-100:-30$ & OVI & $14.10 \pm 0.04 \pm 0.02$ & & & {$[14.18 \pm 0.05 \pm 0.19]$} & & & & \\
\hline & & $\mathrm{Nv}$ & $<13.05$ & & & {$[\mathrm{Ly} \alpha]$} & & & $\mathrm{Nv} / \mathrm{O} \mathrm{vI}$ & $<-1.05$ \\
\hline & & Civ & $13.68 \pm 0.04 \pm 0.01$ & $-56 \pm 5$ & $37 \pm 6$ & $13.65 \pm 0.08 \pm 0.01$ & & & C IV/O vi & $-0.44 \pm 0.10$ \\
\hline & & Si IV & $13.21 \pm 0.03 \pm 0.02$ & $-55 \pm 6$ & $41 \pm 9$ & {$[\operatorname{Ly} \alpha]$} & & & Si IV/C IV & $-0.45 \pm 0.09$ \\
\hline & & Fe III & $14.11 \pm 0.05 \pm 0.02$ & & & & & & $\mathrm{Fe}$ III/C IV & $0.45 \pm 0.10$ \\
\hline & & S III & {$\left[\mathrm{H}_{2}\right]$} & & & & & & & \\
\hline & $-30: 20$ & O VI & $13.94 \pm 0.04 \pm 0.03$ & & & {$[13.80 \pm 0.09 \pm 0.11]$} & & & & \\
\hline & & $\mathrm{Nv}$ & $<13.00$ & & & {$[\mathrm{Ly} \alpha]$} & & & $\mathrm{Nv} / \mathrm{O} \mathrm{vI}$ & $<-0.94$ \\
\hline & & CIV & $13.41 \pm 0.05 \pm 0.02$ & & & $13.42 \pm 0.10 \pm 0.02$ & & & C IV/O VI & $-0.53 \pm 0.12$ \\
\hline & & Si IV & $13.03 \pm 0.04 \pm 0.02$ & & & {$[\operatorname{Ly} \alpha]$} & & & Si IV/C IV & $-0.38 \pm 0.12$ \\
\hline & & Fe III & $13.79 \pm 0.08 \pm 0.04$ & & & & & & $\mathrm{Fe}$ III/C IV & $0.38 \pm 0.14$ \\
\hline & & S III & {$\left[\mathrm{H}_{2}\right]$} & & & & & & & \\
\hline & $20: 70$ & O VI & $13.50 \pm 0.09 \pm 0.04$ & & & {$[13.59 \pm 0.02 \pm 0.01]$} & & & & \\
\hline & & $\mathrm{Nv}$ & $<13.06$ & & & {$[\mathrm{Ly} \alpha]$} & & & $\mathrm{Nv} / \mathrm{O} \mathrm{vI}$ & $<-0.44$ \\
\hline & & Civ & $<12.88$ & & & $<13.25$ & & & $\mathrm{C}$ IV/O VI & $<-0.62$ \\
\hline & & Si IV & $<12.31$ & & & $<12.66$ & & & & \\
\hline & & Fe III & $<13.49$ & & & & & & & \\
\hline & & S III & {$\left[\mathrm{H}_{2}\right]$} & & & & & & & \\
\hline \multirow[t]{6}{*}{$3 \mathrm{C} 273.0$} & $-110: 105$ & $\mathrm{O}$ VI & $14.73 \pm 0.02 \pm 0.01$ & & & {$\left[\mathrm{H}_{2}\right]$} & & & & \\
\hline & & $\mathrm{N} \mathrm{v}$ & $13.94 \pm 0.02 \pm 0.01$ & $-8 \pm 1$ & $94 \pm 4$ & $13.90 \pm 0.03 \pm 0.01$ & $-6 \pm 2$ & $101 \pm 6$ & $\mathrm{~N}$ v/O vi & $-0.81 \pm 0.04$ \\
\hline & & C IV & $14.51 \pm 0.02 \pm 0.01$ & & & $14.50 \pm 0.02 \pm 0.01$ & & & $\mathrm{C}$ IV/O VI & $-0.22 \pm 0.04$ \\
\hline & & Si IV & {$[\operatorname{Ly} \alpha]$} & & & $13.85 \pm 0.02 \pm 0.01$ & $-13 \pm 1$ & $76 \pm 4$ & $\mathrm{Si}$ IV/C IV & $-0.66 \pm 0.04$ \\
\hline & & $\mathrm{Fe}$ III & $14.38 \pm 0.02 \pm 0.02$ & $-9 \pm 7$ & $83 \pm 15$ & & & & $\mathrm{Fe}$ III/C IV & $-0.13 \pm 0.04$ \\
\hline & & S III & $14.63 \pm 0.02 \pm 0.09$ & $-3 \pm 5$ & $88 \pm 8$ & & & & $\mathrm{~S}$ III/C IV & $0.12 \pm 0.04$ \\
\hline \multirow[t]{6}{*}{ 3C 351.0} & $-60: 35$ & $\mathrm{O}$ VI & {$[$ No flux $]$} & & & [No flux $]$ & & & & \\
\hline & & $\mathrm{N} \mathrm{v}$ & $13.63 \pm 0.05 \pm 0.01$ & $-18 \pm 4$ & $43 \pm 9$ & $13.60 \pm 0.09 \pm 0.02$ & & & & \\
\hline & & C IV & $13.96 \pm 0.02 \pm 0.01$ & $-19 \pm 4$ & $54 \pm 4$ & $14.02 \pm 0.04 \pm 0.01$ & $-18 \pm 4$ & $54 \pm 4$ & & \\
\hline & & Si IV & $13.29 \pm 0.02 \pm 0.01$ & $-17 \pm 4$ & $52 \pm 3$ & $13.33 \pm 0.04 \pm 0.04$ & $-18 \pm 13$ & $55 \pm 9$ & $\mathrm{Si}$ IV/C IV & $-0.68 \pm 0.06$ \\
\hline & & Fe III & {$[\mathrm{Ly} \kappa]$} & & & & & & & \\
\hline & & S III & [No flux $]$ & & & & & & & \\
\hline \multirow[t]{23}{*}{ ESO141-G55 } & $-110: 85$ & O vI & $14.51 \pm 0.02 \pm 0.01$ & $0 \pm 2$ & $85 \pm 5$ & $14.58 \pm 0.03 \pm 0.17$ & & & & \\
\hline & & $\mathrm{N} \mathrm{v}$ & $13.67 \pm 0.06 \pm 0.01$ & $-4 \pm 3$ & $95 \pm 8$ & $13.77 \pm 0.08 \pm 0.01$ & $2 \pm 6$ & $93 \pm 12$ & $\mathrm{~N}$ v/O vi & $-0.82 \pm 0.11$ \\
\hline & & C IV & $14.31 \pm 0.06 \pm 0.01$ & & & $14.54 \pm 0.06 \pm 0.01$ & $-8 \pm 2$ & $98 \pm 3$ & $\mathrm{C}$ IV/O VI & $0.11 \pm 0.09$ \\
\hline & & Si IV & $13.82 \pm 0.05 \pm 0.01$ & $-2 \pm 2$ & $86 \pm 5$ & $13.90 \pm 0.05 \pm 0.01$ & $-2 \pm 2$ & $87 \pm 5$ & $\mathrm{Si}$ IV/C IV & $-0.79 \pm 0.11$ \\
\hline & & $\mathrm{Fe}$ III & {$[\mathrm{Fe} \mathrm{II}]$} & & & & & & & \\
\hline & & S III & {$\left[\mathrm{H}_{2}\right]$} & & & & & & & \\
\hline & $-110:-30$ & O VI & $13.83 \pm 0.05 \pm 0.05$ & $-56 \pm 8$ & $35 \pm 7$ & $14.05 \pm 0.05 \pm 0.29$ & & & & \\
\hline & & $\mathrm{Nv}$ & $13.06 \pm 0.14 \pm 0.05$ & $-58 \pm 9$ & $44 \pm 12$ & $<13.33$ & & & $\mathrm{~N}$ v/O vi & $-0.88 \pm 0.16$ \\
\hline & & C IV & $13.84 \pm 0.07 \pm 0.01$ & $-55 \pm 7$ & $39 \pm 9$ & $14.00 \pm 0.06 \pm 0.01$ & $-62 \pm 7$ & $45 \pm 6$ & $\mathrm{C}$ IV/O VI & $0.14 \pm 0.11$ \\
\hline & & Si IV & $13.13 \pm 0.06 \pm 0.02$ & & & $13.19 \pm 0.09 \pm 0.03$ & & & $\mathrm{Si}$ IV/C IV & $-0.92 \pm 0.14$ \\
\hline & & Fe III & {$[\mathrm{Fe} \mathrm{II}]$} & & & & & & & \\
\hline & & S III & {$\left[\mathrm{H}_{2}\right]$} & & & & & & & \\
\hline & $-30: 30$ & O vI & $14.30 \pm 0.03 \pm 0.02$ & $4 \pm 5$ & $32 \pm 2$ & $14.26 \pm 0.03 \pm 0.07$ & & & & \\
\hline & & $\mathrm{N} \mathrm{v}$ & $13.42 \pm 0.07 \pm 0.02$ & & & $13.48 \pm 0.09 \pm 0.03$ & & & $\mathrm{~N}$ v/O vi & $-0.83 \pm 0.12$ \\
\hline & & C IV & $14.00 \pm 0.11 \pm 0.02$ & $0 \pm 8$ & $39 \pm 2$ & $14.28 \pm 0.09 \pm 0.01$ & $1 \pm 5$ & $34 \pm 2$ & $\mathrm{C}$ IV/O VI & $0.14 \pm 0.15$ \\
\hline & & Si IV & $13.60 \pm 0.08 \pm 0.01$ & $1 \pm 5$ & $34 \pm 2$ & $13.69 \pm 0.06 \pm 0.01$ & $0 \pm 5$ & $33 \pm 2$ & $\mathrm{Si}$ IV/C IV & $-0.78 \pm 0.17$ \\
\hline & & Fe III & $14.21 \pm 0.03 \pm 0.03$ & $3 \pm 4$ & $28 \pm 3$ & & & & $\mathrm{Fe}$ III/C IV & $-0.21 \pm 0.14$ \\
\hline & & S III & {$\left[\mathrm{H}_{2}\right]$} & & & & & & & \\
\hline & $30: 85$ & O VI & $13.84 \pm 0.04 \pm 0.06$ & & & $13.99 \pm 0.05 \pm 0.12$ & & & & \\
\hline & & $\mathrm{N} \mathrm{v}$ & $13.00 \pm 0.13 \pm 0.05$ & & & $13.24 \pm 0.13 \pm 0.05$ & & & $\mathrm{~N}$ v/O vi & $-0.79 \pm 0.19$ \\
\hline & & C IV & $13.63 \pm 0.08 \pm 0.03$ & & & $13.81 \pm 0.08 \pm 0.03$ & & & $\mathrm{C}$ IV/O VI & $-0.01 \pm 0.13$ \\
\hline & & Si IV & $13.15 \pm 0.07 \pm 0.03$ & & & $13.22 \pm 0.08 \pm 0.03$ & & & Si IV/C IV & $-0.71 \pm 0.16$ \\
\hline & & $\begin{array}{l}\text { Fe III } \\
S_{\text {III }}\end{array}$ & $\begin{array}{c}13.72 \pm 0.06 \pm 0.08 \\
{\left[\mathrm{H}_{2}\right]}\end{array}$ & $46 \pm 6$ & $21 \pm 5$ & & & & $\mathrm{Fe}$ III/C IV & $-0.18 \pm 0.13$ \\
\hline \multirow[t]{2}{*}{ Fairall9 } & $-60: 70$ & O VI & $14.18 \pm 0.10 \pm 0.04$ & & & {$\left[\mathrm{H}_{2}\right]$} & & & & \\
\hline & & $\mathrm{Nv}$ & $<13.34$ & & & $<13.62$ & & & $\mathrm{~N}$ v/O vI & $<-0.84$ \\
\hline
\end{tabular}


Table 2

(Continuted)

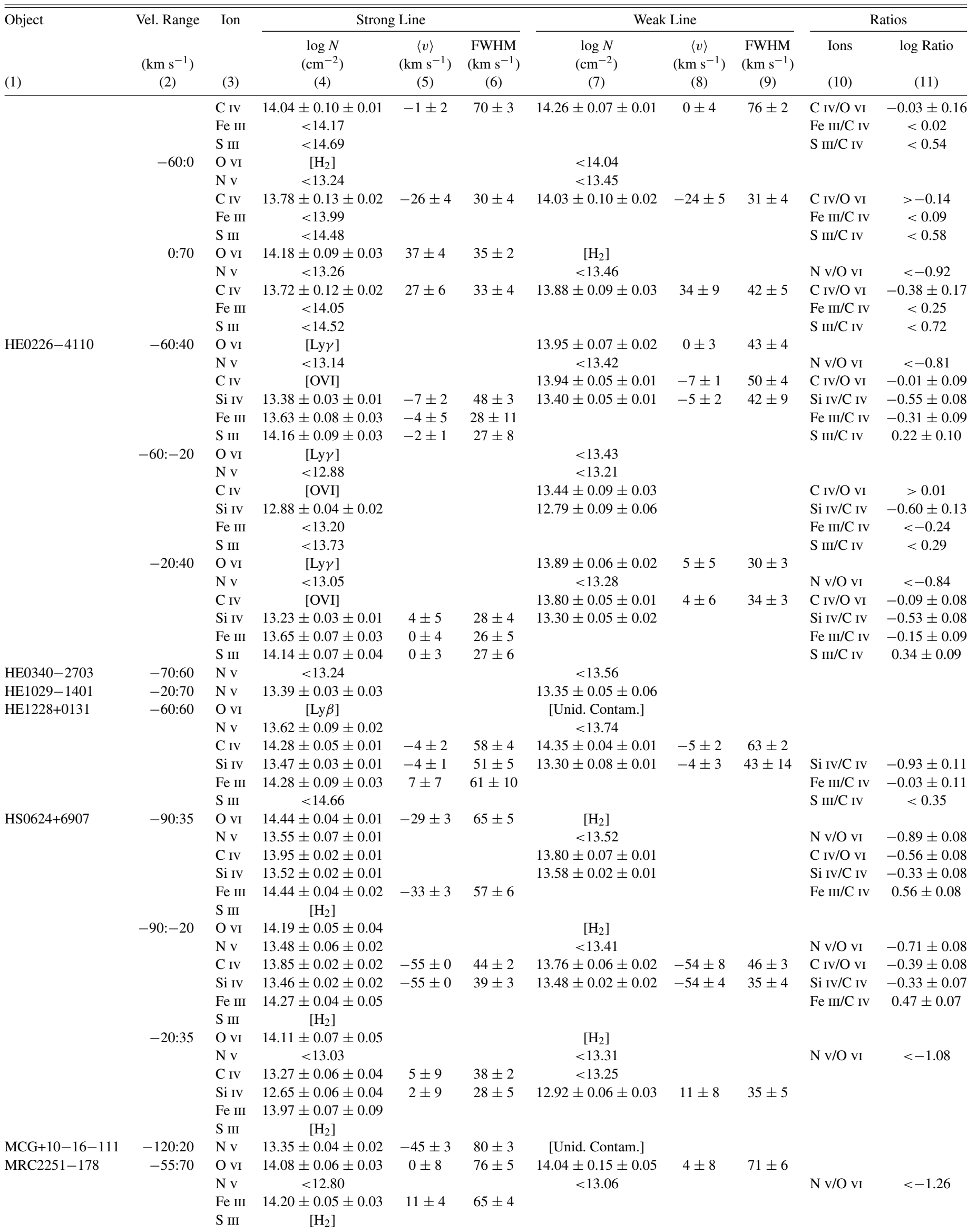


Table 2

(Continuted)

\begin{tabular}{|c|c|c|c|c|c|c|c|c|c|c|}
\hline \multirow{2}{*}{$\begin{array}{l}\text { Object } \\
\text { (1) }\end{array}$} & \multirow{2}{*}{$\begin{array}{l}\text { Vel. Range } \\
\left(\mathrm{km} \mathrm{s}^{-1}\right) \\
(2)\end{array}$} & \multirow{2}{*}{$\begin{array}{l}\text { Ion } \\
\text { (3) }\end{array}$} & \multicolumn{3}{|c|}{ Strong Line } & \multicolumn{3}{|c|}{ Weak Line } & \multicolumn{2}{|c|}{ Ratios } \\
\hline & & & $\begin{array}{c}\log N \\
\left(\mathrm{~cm}^{-2}\right) \\
(4)\end{array}$ & $\begin{array}{c}\langle v\rangle \\
\left(\mathrm{km} \mathrm{s}^{-1}\right) \\
(5)\end{array}$ & $\begin{array}{c}\text { FWHM } \\
\left(\mathrm{km} \mathrm{s}^{-1}\right) \\
(6)\end{array}$ & $\begin{array}{c}\log N \\
\left(\mathrm{~cm}^{-2}\right) \\
(7)\end{array}$ & $\begin{array}{c}\langle v\rangle \\
\left(\mathrm{km} \mathrm{s}^{-1}\right) \\
(8)\end{array}$ & $\begin{array}{c}\text { FWHM } \\
\left(\mathrm{km} \mathrm{s}^{-1}\right) \\
(9)\end{array}$ & $\begin{array}{l}\text { Ions } \\
\text { (10) }\end{array}$ & $\begin{array}{c}\log \text { Ratio } \\
\text { (11) }\end{array}$ \\
\hline Mrk110 & $-70: 60$ & $\mathrm{Nv}$ & $13.37 \pm 0.10 \pm 0.03$ & & & $<13.47$ & & & & \\
\hline \multirow[t]{18}{*}{ Mrk205 } & $-95: 50$ & O VI & $14.16 \pm 0.04 \pm 0.02$ & & & $14.21 \pm 0.06 \pm 0.03$ & & & & \\
\hline & & $\mathrm{N} \mathrm{v}$ & $13.26 \pm 0.12 \pm 0.01$ & & & $<13.55$ & & & & \\
\hline & & $\mathrm{C}$ IV & $14.11 \pm 0.03 \pm 0.01$ & & & $14.07 \pm 0.05 \pm 0.01$ & & & $\mathrm{C}$ IV/O VI & $-0.10 \pm 0.09$ \\
\hline & & Si IV & $13.57 \pm 0.02 \pm 0.01$ & & & $13.55 \pm 0.04 \pm 0.01$ & & & $\mathrm{Si}$ IV/C IV & $-0.53 \pm 0.07$ \\
\hline & & $\mathrm{Fe}$ III & $14.47 \pm 0.03 \pm 0.01$ & & & & & & $\mathrm{Fe}$ III/C IV & $0.38 \pm 0.07$ \\
\hline & & $\mathrm{S}_{\mathrm{III}}$ & $14.72 \pm 0.04 \pm 0.07$ & & & & & & $\mathrm{~S}$ III/C IV & $0.63 \pm 0.07$ \\
\hline & $-95:-30$ & O VI & $13.87 \pm 0.05 \pm 0.04$ & & & $14.03 \pm 0.06 \pm 0.05$ & & & & \\
\hline & & $\mathrm{N} \mathrm{v}$ & $13.21 \pm 0.09 \pm 0.01$ & $-56 \pm 3$ & $31 \pm 9$ & $<13.34$ & & & $\mathrm{~N}$ v/O vI & $-0.74 \pm 0.12$ \\
\hline & & $\mathrm{C}_{\mathrm{IV}}$ & $13.93 \pm 0.03 \pm 0.01$ & & & $13.81 \pm 0.07 \pm 0.01$ & $-56 \pm 7$ & $39 \pm 4$ & $\mathrm{C}$ IV/O VI & $-0.08 \pm 0.11$ \\
\hline & & Si IV & $13.40 \pm 0.03 \pm 0.01$ & $-57 \pm 5$ & $32 \pm 5$ & $13.37 \pm 0.03 \pm 0.01$ & $-51 \pm 4$ & $27 \pm 10$ & Si IV/C IV & $-0.49 \pm 0.09$ \\
\hline & & $\mathrm{Fe}$ III & $14.34 \pm 0.03 \pm 0.02$ & $-58 \pm 4$ & $26 \pm 5$ & & & & $\mathrm{Fe}$ III/C IV & $0.47 \pm 0.09$ \\
\hline & & $S_{\text {III }}$ & $14.49 \pm 0.05 \pm 0.06$ & $-57 \pm 6$ & $28 \pm 5$ & & & & $\mathrm{~S}$ III/C IV & $0.62 \pm 0.09$ \\
\hline & $-30: 50$ & O VI & $13.86 \pm 0.05 \pm 0.04$ & $4 \pm 6$ & $45 \pm 2$ & $13.74 \pm 0.11 \pm 0.09$ & $2 \pm 7$ & $40 \pm 6$ & & \\
\hline & & $\mathrm{Nv}$ & $<13.09$ & & & $<13.40$ & & & $\mathrm{~N}$ v/O vI & $<-0.71$ \\
\hline & & C IV & $13.66 \pm 0.04 \pm 0.02$ & $0 \pm 8$ & $41 \pm 5$ & $13.73 \pm 0.08 \pm 0.02$ & & & $\mathrm{C}$ IV/O VI & $-0.10 \pm 0.15$ \\
\hline & & Si IV & $13.12 \pm 0.04 \pm 0.01$ & & & $13.10 \pm 0.07 \pm 0.02$ & & & $\mathrm{Si}$ IV/C IV & $-0.59 \pm 0.12$ \\
\hline & & $\mathrm{Fe}$ III & $13.90 \pm 0.05 \pm 0.05$ & & & & & & $\mathrm{Fe}$ III/C IV & $0.20 \pm 0.10$ \\
\hline & & S III & $14.37 \pm 0.07 \pm 0.17$ & & & & & & $\mathrm{~S}$ III/C IV & $0.67 \pm 0.11$ \\
\hline \multirow[t]{30}{*}{ Mrk279 } & $-110: 65$ & $\mathrm{O}$ VI & $14.41 \pm 0.02 \pm 0.01$ & $-24 \pm 3$ & $91 \pm 4$ & $14.41 \pm 0.02 \pm 0.01$ & $-26 \pm 3$ & $88 \pm 5$ & & \\
\hline & & $\mathrm{N} \mathrm{v}$ & $13.26 \pm 0.05 \pm 0.01$ & $-6 \pm 3$ & $77 \pm 7$ & $13.35 \pm 0.07 \pm 0.01$ & $-14 \pm 5$ & $89 \pm 10$ & & \\
\hline & & C IV & $14.24 \pm 0.02 \pm 0.01$ & $-25 \pm 1$ & $74 \pm 4$ & $14.20 \pm 0.02 \pm 0.01$ & $-30 \pm 1$ & $70 \pm 4$ & & \\
\hline & & Si IV & $13.66 \pm 0.02 \pm 0.01$ & $-24 \pm 1$ & $62 \pm 4$ & $13.66 \pm 0.02 \pm 0.01$ & $-23 \pm 2$ & $78 \pm 3$ & & \\
\hline & & $\mathrm{Fe}$ III & $14.33 \pm 0.02 \pm 0.01$ & $-33 \pm 2$ & $61 \pm 6$ & & & & & \\
\hline & & S III & $14.55 \pm 0.02 \pm 0.04$ & $-32 \pm 1$ & $57 \pm 8$ & & & & & \\
\hline & $-110:-65$ & O VI & $13.67 \pm 0.02 \pm 0.04$ & & & $13.67 \pm 0.04 \pm 0.05$ & & & & \\
\hline & & $\mathrm{N} v$ & $<12.43$ & & & $<12.71$ & & & $\mathrm{~N}$ v/O vI & $<-1.24$ \\
\hline & & C IV & $13.19 \pm 0.04 \pm 0.04$ & & & $13.24 \pm 0.07 \pm 0.04$ & & & $\mathrm{C}$ IV/O VI & $-0.46 \pm 0.09$ \\
\hline & & Si IV & $12.40 \pm 0.05 \pm 0.05$ & & & $12.57 \pm 0.09 \pm 0.04$ & & & $\mathrm{Si}$ IV/C IV & $-0.73 \pm 0.13$ \\
\hline & & $\mathrm{Fe}$ III & $13.37 \pm 0.05 \pm 0.08$ & & & & & & $\mathrm{Fe}$ III/C IV & $0.16 \pm 0.09$ \\
\hline & & $S_{\text {III }}$ & $13.58 \pm 0.08 \pm 0.09$ & & & & & & $\mathrm{~S}$ III/C IV & $0.37 \pm 0.11$ \\
\hline & $-65:-20$ & O VI & $13.95 \pm 0.02 \pm 0.03$ & & & $13.98 \pm 0.02 \pm 0.03$ & & & & \\
\hline & & $\mathrm{N} v$ & $12.79 \pm 0.06 \pm 0.03$ & & & $12.84 \pm 0.09 \pm 0.03$ & & & $\mathrm{~N}$ v/O vI & $-1.15 \pm 0.11$ \\
\hline & & C IV & $13.97 \pm 0.02 \pm 0.01$ & & & $13.93 \pm 0.02 \pm 0.02$ & & & $\mathrm{C}_{\mathrm{IV} / \mathrm{O}} \mathrm{VI}$ & $-0.01 \pm 0.04$ \\
\hline & & Si IV & $13.43 \pm 0.02 \pm 0.01$ & $-38 \pm 5$ & $26 \pm 4$ & $13.39 \pm 0.02 \pm 0.01$ & & & Si IV/C IV & $-0.54 \pm 0.04$ \\
\hline & & Fe III & $14.10 \pm 0.02 \pm 0.02$ & & & & & & $\mathrm{Fe}$ III/C IV & $0.15 \pm 0.04$ \\
\hline & & $S_{\text {III }}$ & $14.32 \pm 0.02 \pm 0.03$ & & & & & & $\mathrm{~S}$ III/C IV & $0.37 \pm 0.04$ \\
\hline & $-20: 20$ & O VI & $13.98 \pm 0.02 \pm 0.03$ & & & $13.98 \pm 0.02 \pm 0.03$ & & & & \\
\hline & & $\mathrm{N} v$ & $12.93 \pm 0.04 \pm 0.03$ & & & $13.01 \pm 0.06 \pm 0.03$ & & & $\mathrm{~N}$ v/O vi & $-1.01 \pm 0.08$ \\
\hline & & C IV & $13.74 \pm 0.02 \pm 0.03$ & & & $13.74 \pm 0.02 \pm 0.02$ & & & $\mathrm{C}$ IV/O VI & $-0.24 \pm 0.04$ \\
\hline & & Si IV & $13.20 \pm 0.02 \pm 0.03$ & & & $13.13 \pm 0.02 \pm 0.03$ & & & Si IV/C IV & $-0.58 \pm 0.04$ \\
\hline & & $\mathrm{Fe}$ III & $13.79 \pm 0.02 \pm 0.04$ & & & & & & $\mathrm{Fe}$ III/C IV & $0.05 \pm 0.04$ \\
\hline & & S III & $14.10 \pm 0.03 \pm 0.05$ & & & & & & $\mathrm{~S}$ III/C IV & $0.36 \pm 0.04$ \\
\hline & $20: 65$ & O VI & $13.60 \pm 0.03 \pm 0.04$ & & & $13.49 \pm 0.06 \pm 0.06$ & & & & \\
\hline & & $\mathrm{N} v$ & $12.64 \pm 0.08 \pm 0.03$ & & & $<12.69$ & & & $\mathrm{~N}$ v/O vi & $-0.90 \pm 0.11$ \\
\hline & & C IV & $13.20 \pm 0.03 \pm 0.03$ & & & {$[12.88 \pm 0.13 \pm 0.05]$} & & & $\mathrm{C}_{\mathrm{IV} / \mathrm{O}} \mathrm{VI}$ & $-0.34 \pm 0.08$ \\
\hline & & Si IV & $12.43 \pm 0.05 \pm 0.05$ & & & {$[12.70 \pm 0.07 \pm 0.03]$} & & & $\mathrm{Si}$ IV/C IV & $-0.77 \pm 0.06$ \\
\hline & & $\mathrm{Fe}$ III & $12.84 \pm 0.14 \pm 0.12$ & & & & & & $\mathrm{Fe}$ III/C IV & $-0.36 \pm 0.14$ \\
\hline & & $S_{\text {III }}$ & $<13.33$ & & & & & & S III/C IV & $<0.13$ \\
\hline \multirow[t]{4}{*}{ Mrk290 } & $-65: 60$ & $\mathrm{O}$ VI & $14.15 \pm 0.03 \pm 0.02$ & $-15 \pm 6$ & $66 \pm 4$ & $14.16 \pm 0.06 \pm 0.03$ & $-13 \pm 6$ & $65 \pm 5$ & & \\
\hline & & $\mathrm{N} \mathrm{v}$ & $<13.35$ & & & [Emission] & & & $\mathrm{N}$ v/O vi & $<-0.80$ \\
\hline & & $\mathrm{Fe}$ III & $14.02 \pm 0.06 \pm 0.03$ & $-16 \pm 3$ & $57 \pm 7$ & & & & & \\
\hline & & $S_{\text {III }}$ & $14.37 \pm 0.07 \pm 0.16$ & & & & & & & \\
\hline \multirow[t]{9}{*}{ Mrk335 } & $-95: 60$ & O VI & $14.07 \pm 0.02 \pm 0.02$ & $-10 \pm 3$ & $76 \pm 4$ & $14.01 \pm 0.06 \pm 0.23$ & $-8 \pm 2$ & $77 \pm 8$ & & \\
\hline & & $\mathrm{N} \mathrm{v}$ & $<13.14$ & & & $<13.26$ & & & $\mathrm{~N}$ v/O vI & $<-0.90$ \\
\hline & & C IV & $14.17 \pm 0.03 \pm 0.01$ & $-3 \pm 2$ & $76 \pm 4$ & $14.18 \pm 0.07 \pm 0.01$ & $-3 \pm 4$ & $69 \pm 16$ & $\mathrm{C}$ IV/O VI & $0.14 \pm 0.10$ \\
\hline & & Si IV & $13.61 \pm 0.03 \pm 0.01$ & $-4 \pm 1$ & $68 \pm 4$ & $13.56 \pm 0.05 \pm 0.01$ & $-11 \pm 4$ & $75 \pm 9$ & $\mathrm{Si}$ IV/C IV & $-0.59 \pm 0.10$ \\
\hline & & $\mathrm{Fe}$ III & $14.27 \pm 0.03 \pm 0.02$ & $-9 \pm 2$ & $73 \pm 4$ & & & & $\mathrm{Fe}$ III/C IV & $0.09 \pm 0.09$ \\
\hline & & $\mathrm{S}_{\text {III }}$ & $14.53 \pm 0.03 \pm 0.26$ & & & & & & & \\
\hline & $-95:-40$ & O VI & $13.32 \pm 0.06 \pm 0.12$ & & & $13.27 \pm 0.13 \pm 0.42$ & & & & \\
\hline & & $\mathrm{N} \mathrm{v}$ & $<12.88$ & & & $<13.03$ & & & $\mathrm{~N}$ v/O vI & $<-0.41$ \\
\hline & & $\mathrm{C}_{\text {IV }}$ & $13.26 \pm 0.09 \pm 0.02$ & & & $<13.55$ & & & $\mathrm{C}$ IV/O VI & $-0.03 \pm 0.17$ \\
\hline
\end{tabular}


Table 2

(Continuted)

\begin{tabular}{|c|c|c|c|c|c|c|c|c|c|c|}
\hline \multirow[t]{2}{*}{ Object } & \multirow{2}{*}{$\begin{array}{c}\text { Vel. Range } \\
\left(\mathrm{km} \mathrm{s}^{-1}\right)\end{array}$} & \multirow{2}{*}{$\begin{array}{l}\text { Ion } \\
\text { (3) }\end{array}$} & \multicolumn{3}{|c|}{ Strong Line } & \multicolumn{3}{|c|}{ Weak Line } & \multicolumn{2}{|c|}{ Ratios } \\
\hline & & & $\begin{array}{c}\log N \\
\left(\mathrm{~cm}^{-2}\right) \\
(4)\end{array}$ & $\begin{array}{c}\langle v\rangle \\
\left(\mathrm{km} \mathrm{s}^{-1}\right) \\
(5)\end{array}$ & $\begin{array}{c}\text { FWHM } \\
\left(\mathrm{km} \mathrm{s}^{-1}\right) \\
(6)\end{array}$ & $\begin{array}{c}\log N \\
\left(\mathrm{~cm}^{-2}\right) \\
(7)\end{array}$ & $\begin{array}{c}\langle v\rangle \\
\left(\mathrm{km} \mathrm{s}^{-1}\right) \\
(8)\end{array}$ & $\begin{array}{c}\text { FWHM } \\
\left(\mathrm{km} \mathrm{s}^{-1}\right) \\
(9)\end{array}$ & $\begin{array}{l}\text { Ions } \\
\text { (10) }\end{array}$ & $\begin{array}{c}\log \text { Ratio } \\
\text { (11) }\end{array}$ \\
\hline \multirow{9}{*}{ (1) } & & Si IV & $12.68 \pm 0.10 \pm 0.02$ & & & $<12.88$ & & & Si IV/C IV & $-0.58 \pm 0.13$ \\
\hline & & $\mathrm{Fe}$ III & $13.54 \pm 0.06 \pm 0.11$ & & & & & & & \\
\hline & & S III & $<13.56$ & & & & & & $\mathrm{~S}$ III/C IV & $<0.30$ \\
\hline & $-40: 60$ & $\mathrm{O}$ VI & $13.98 \pm 0.02 \pm 0.03$ & $2 \pm 3$ & $51 \pm 2$ & $13.93 \pm 0.05 \pm 0.13$ & & & & \\
\hline & & $\mathrm{N} \mathrm{v}$ & $<13.04$ & & & $<13.07$ & & & $\mathrm{~N}$ v/O vI & $<-0.92$ \\
\hline & & C IV & $14.12 \pm 0.03 \pm 0.01$ & $6 \pm 3$ & $54 \pm 3$ & $14.18 \pm 0.07 \pm 0.01$ & $2 \pm 3$ & $57 \pm 3$ & $\mathrm{C}$ IV/O VI & $0.19 \pm 0.09$ \\
\hline & & Si IV & $13.56 \pm 0.03 \pm 0.01$ & $3 \pm 2$ & $51 \pm 4$ & $13.47 \pm 0.05 \pm 0.01$ & $2 \pm 4$ & $49 \pm 7$ & Si IV/C IV & $-0.63 \pm 0.10$ \\
\hline & & Fe III & $14.18 \pm 0.02 \pm 0.03$ & $1 \pm 4$ & $53 \pm 5$ & & & & $\mathrm{Fe}$ III/C IV & $0.03 \pm 0.08$ \\
\hline & & S III & $14.50 \pm 0.03 \pm 0.25$ & & & & & & & \\
\hline \multirow[t]{4}{*}{ Mrk421 } & $-100: 60$ & $\mathrm{O}$ VI & $14.33 \pm 0.02 \pm 0.01$ & $-18 \pm 4$ & $94 \pm 2$ & $14.36 \pm 0.02 \pm 0.01$ & $-16 \pm 3$ & $91 \pm 2$ & & \\
\hline & & $\mathrm{N} \mathrm{v}$ & $<13.11$ & & & $<13.40$ & & & $\mathrm{~N}$ v/O vi & $<-1.24$ \\
\hline & & Fe III & $14.33 \pm 0.02 \pm 0.01$ & $-41 \pm 3$ & $76 \pm 5$ & & & & & \\
\hline & & S III & $14.50 \pm 0.03 \pm 0.04$ & $-35 \pm 2$ & $69 \pm 4$ & & & & & \\
\hline \multirow[t]{12}{*}{ Mrk478 } & $-120: 65$ & O VI & $14.47 \pm 0.05 \pm 0.01$ & $-37 \pm 4$ & $98 \pm 6$ & {$[14.25 \pm 0.11 \pm 0.04]$} & & & & \\
\hline & & $\mathrm{N} \mathrm{v}$ & $13.55 \pm 0.02 \pm 0.01$ & $-48 \pm 4$ & $93 \pm 8$ & {$[13.36 \pm 0.06 \pm 0.03]$} & & & $\mathrm{N}$ v/O vi & $-0.92 \pm 0.05$ \\
\hline & & Fe III & $<13.98$ & & & & & & & \\
\hline & & S III & $<14.44$ & & & & & & & \\
\hline & $-120:-10$ & $\mathrm{O}_{\mathrm{VI}}$ & $14.35 \pm 0.05 \pm 0.02$ & & & {$[14.04 \pm 0.13 \pm 0.07]$} & & & & \\
\hline & & $\mathrm{N} \mathrm{v}$ & $13.48 \pm 0.02 \pm 0.02$ & $-62 \pm 3$ & $61 \pm 3$ & {$[13.35 \pm 0.05 \pm 0.03]$} & & & $\mathrm{N}$ v/O vI & $-0.87 \pm 0.05$ \\
\hline & & $\mathrm{Fe}$ III & $13.98 \pm 0.10 \pm 0.05$ & & & & & & & \\
\hline & & S III & $14.32 \pm 0.13 \pm 0.06$ & & & & & & & \\
\hline & $-10: 65$ & O VI & $13.87 \pm 0.09 \pm 0.06$ & & & {$[13.85 \pm 0.15 \pm 0.09]$} & & & & \\
\hline & & $\mathrm{N} \mathrm{v}$ & $<12.76$ & & & $<12.82$ & & & $\mathrm{~N}$ v/O vi & $<-1.11$ \\
\hline & & Fe III & $<13.72$ & & & & & & & \\
\hline & & S III & $<14.18$ & & & & & & & \\
\hline \multirow[t]{4}{*}{ Mrk501 } & $-55: 25$ & O VI & $14.23 \pm 0.05 \pm 0.03$ & $-16 \pm 5$ & $44 \pm 3$ & $14.22 \pm 0.07 \pm 0.05$ & $-25 \pm 8$ & $46 \pm 6$ & & \\
\hline & & $\mathrm{N} \mathrm{v}$ & $<13.29$ & & & $<13.56$ & & & $\mathrm{~N}$ v/O vi & $<-0.93$ \\
\hline & & Fe III & $13.94 \pm 0.09 \pm 0.04$ & $-11 \pm 3$ & $37 \pm 4$ & & & & & \\
\hline & & S III & $14.42 \pm 0.08 \pm 0.04$ & $-19 \pm 5$ & $39 \pm 5$ & & & & & \\
\hline \multirow[t]{24}{*}{ Mrk509 } & $-90: 115$ & O VI & $14.67 \pm 0.02 \pm 0.01$ & $21 \pm 2$ & $102 \pm 2$ & $14.72 \pm 0.02 \pm 0.02$ & $15 \pm 7$ & $115 \pm 8$ & & \\
\hline & & $\mathrm{N} \mathrm{v}$ & $13.81 \pm 0.04 \pm 0.01$ & $32 \pm 6$ & $101 \pm 10$ & $13.76 \pm 0.07 \pm 0.01$ & $26 \pm 7$ & $95 \pm 12$ & $\mathrm{~N}$ v/O vI & $-0.91 \pm 0.09$ \\
\hline & & C IV & $14.51 \pm 0.03 \pm 0.01$ & & & $14.37 \pm 0.04 \pm 0.01$ & $38 \pm 5$ & $73 \pm 10$ & $\mathrm{C}$ IV/O VI & $-0.26 \pm 0.06$ \\
\hline & & Si IV & $13.89 \pm 0.02 \pm 0.01$ & $33 \pm 1$ & $82 \pm 4$ & $13.89 \pm 0.02 \pm 0.01$ & $36 \pm 2$ & $86 \pm 6$ & Si IV/C IV & $-0.55 \pm 0.06$ \\
\hline & & Fe III & $14.51 \pm 0.02 \pm 0.01$ & & & & & & & \\
\hline & & S III & $14.99 \pm 0.02 \pm 0.25$ & & & & & & & \\
\hline & $-90:-30$ & $\mathrm{O}$ VI & $13.81 \pm 0.03 \pm 0.05$ & & & {$\left[\mathrm{H}_{2}\right]$} & & & & \\
\hline & & $\mathrm{N} \mathrm{v}$ & $<12.89$ & & & $<13.17$ & & & $\mathrm{~N}$ v/O vI & $<-0.92$ \\
\hline & & C IV & $13.14 \pm 0.09 \pm 0.03$ & & & $<13.37$ & & & C IV/O VI & $-0.67 \pm 0.09$ \\
\hline & & Si IV & $<12.45$ & & & $<12.70$ & & & $\mathrm{Si}$ IV/C IV & $<-0.69$ \\
\hline & & $\mathrm{Fe}$ III & [Unid. Contam.] & & & & & & & \\
\hline & & S III & $<13.53$ & & & & & & $\mathrm{~S}$ III/C IV & $<0.39$ \\
\hline & $-30: 45$ & $\mathrm{O}_{\mathrm{VI}}$ & $14.43 \pm 0.02 \pm 0.02$ & & & $14.43 \pm 0.02 \pm 0.03$ & & & & \\
\hline & & $\mathrm{N} \mathrm{v}$ & $13.51 \pm 0.04 \pm 0.02$ & & & $13.54 \pm 0.07 \pm 0.01$ & & & $\mathrm{~N}$ v/O vI & $-0.90 \pm 0.09$ \\
\hline & & C IV & $14.30 \pm 0.04 \pm 0.01$ & $12 \pm 4$ & $42 \pm 4$ & $14.16 \pm 0.05 \pm 0.01$ & $16 \pm 5$ & $35 \pm 10$ & C IV/O VI & $-0.20 \pm 0.07$ \\
\hline & & Si IV & $13.68 \pm 0.03 \pm 0.01$ & $14 \pm 4$ & $39 \pm 5$ & $13.66 \pm 0.03 \pm 0.01$ & $16 \pm 4$ & $39 \pm 8$ & Si IV/C IV & $-0.56 \pm 0.07$ \\
\hline & & Fe III & $14.29 \pm 0.02 \pm 0.02$ & $13 \pm 4$ & $37 \pm 3$ & & & & $\mathrm{Fe}$ III/C IV & $0.06 \pm 0.06$ \\
\hline & & S III & $14.71 \pm 0.02 \pm 0.07$ & & & & & & & \\
\hline & $45: 115$ & $\mathrm{O}$ VI & $14.16 \pm 0.02 \pm 0.03$ & & & $14.19 \pm 0.02 \pm 0.04$ & & & & \\
\hline & & $\mathrm{N} \mathrm{v}$ & $13.46 \pm 0.04 \pm 0.02$ & & & $13.29 \pm 0.11 \pm 0.02$ & & & $\mathrm{~N}$ v/O vi & $-0.80 \pm 0.12$ \\
\hline & & C IV & $14.06 \pm 0.03 \pm 0.01$ & $76 \pm 6$ & $39 \pm 4$ & $14.01 \pm 0.05 \pm 0.01$ & $70 \pm 6$ & $34 \pm 8$ & $\mathrm{C}$ IV/O vi & $-0.14 \pm 0.07$ \\
\hline & & Si IV & $13.46 \pm 0.03 \pm 0.01$ & $72 \pm 6$ & $38 \pm 4$ & $13.49 \pm 0.04 \pm 0.01$ & $74 \pm 7$ & $36 \pm 6$ & $\mathrm{Si}$ IV/C IV & $-0.57 \pm 0.08$ \\
\hline & & $\mathrm{Fe}$ III & $13.87 \pm 0.04 \pm 0.06$ & & & & & & & \\
\hline & & S III & $14.67 \pm 0.02 \pm 0.30$ & & & & & & & \\
\hline Mrk771 & $-40: 70$ & O VI & {$[\operatorname{Ly} \beta]$} & & & $14.25 \pm 0.17 \pm 0.03$ & $12 \pm 6$ & $62 \pm 9$ & & \\
\hline & & $\mathrm{N} \mathrm{v}$ & $13.40 \pm 0.04 \pm 0.02$ & $15 \pm 0$ & $65 \pm 3$ & $13.63 \pm 0.05 \pm 0.02$ & & & $\mathrm{~N}$ v/O vI & $-0.73 \pm 0.18$ \\
\hline & & $\mathrm{Fe}$ III & $<14.29$ & & & & & & & \\
\hline & & S III & $<14.78$ & & & & & & & \\
\hline Mrk817 & $-70: 60$ & $\mathrm{O}$ VI & $14.36 \pm 0.02 \pm 0.02$ & & & $14.38 \pm 0.02 \pm 0.02$ & & & & \\
\hline & & $\mathrm{N} \mathrm{v}$ & {$[\operatorname{Ly} \alpha]$} & & & $<13.16$ & & & $\mathrm{~N}$ v/O vi & $<-1.21$ \\
\hline & & Fe III & $14.02 \pm 0.02 \pm 0.03$ & $-15 \pm 4$ & $66 \pm 6$ & & & & & \\
\hline & & S III & $14.31 \pm 0.02 \pm 0.05$ & $-19 \pm 3$ & $54 \pm 6$ & & & & & \\
\hline Mrk876 & $-80: 80$ & $\mathrm{O}$ VI & $14.42 \pm 0.02 \pm 0.01$ & & & {$[\operatorname{Ly} \beta]$} & & & & \\
\hline & & $\mathrm{N} \mathrm{v}$ & $13.45 \pm 0.09 \pm 0.01$ & & & $<13.80$ & & & $\mathrm{~N}$ v/O vI & $-0.97 \pm 0.09$ \\
\hline
\end{tabular}


Table 2

(Continuted)

\begin{tabular}{|c|c|c|c|c|c|c|c|c|c|c|}
\hline \multirow{2}{*}{$\begin{array}{l}\text { Object } \\
\text { (1) }\end{array}$} & \multirow{2}{*}{$\begin{array}{l}\text { Vel. Range } \\
\left(\mathrm{km} \mathrm{s}^{-1}\right) \\
(2)\end{array}$} & \multirow{2}{*}{$\begin{array}{l}\text { Ion } \\
\text { (3) }\end{array}$} & \multicolumn{3}{|c|}{ Strong Line } & \multicolumn{3}{|c|}{ Weak Line } & \multicolumn{2}{|c|}{ Ratios } \\
\hline & & & $\begin{array}{c}\log N \\
\left(\mathrm{~cm}^{-2}\right) \\
(4)\end{array}$ & $\begin{array}{c}\langle v\rangle \\
\left(\mathrm{km} \mathrm{s}^{-1}\right) \\
(5)\end{array}$ & $\begin{array}{c}\text { FWHM } \\
\left(\mathrm{km} \mathrm{s}^{-1}\right) \\
(6)\end{array}$ & $\begin{array}{c}\log N \\
\left(\mathrm{~cm}^{-2}\right) \\
(7)\end{array}$ & $\begin{array}{c}\langle v\rangle \\
\left(\mathrm{km} \mathrm{s}^{-1}\right) \\
(8)\end{array}$ & $\begin{array}{c}\text { FWHM } \\
\left(\mathrm{km} \mathrm{s}^{-1}\right) \\
(9)\end{array}$ & $\begin{array}{l}\text { Ions } \\
\text { (10) }\end{array}$ & $\begin{array}{c}\log \text { Ratio } \\
\text { (11) }\end{array}$ \\
\hline & & C IV & $14.21 \pm 0.02 \pm 0.01$ & & & $14.14 \pm 0.04 \pm 0.01$ & & & $\mathrm{C}$ IV/O VI & $-0.24 \pm 0.04$ \\
\hline & & Si IV & $13.65 \pm 0.02 \pm 0.01$ & & & $13.60 \pm 0.02 \pm 0.01$ & & & $\mathrm{Si}$ IV/C IV & $-0.56 \pm 0.05$ \\
\hline & & $\mathrm{Fe}$ III & $14.24 \pm 0.03 \pm 0.02$ & $-13 \pm 4$ & $68 \pm 7$ & & & & $\mathrm{Fe}$ III/C IV & $0.06 \pm 0.05$ \\
\hline & & $S_{\text {III }}$ & {$[14.51 \pm 0.03 \pm 0.31]$} & & & & & & & \\
\hline & $-80:-30$ & O VI & $13.86 \pm 0.02 \pm 0.05$ & & & {$[\operatorname{Ly} \beta]$} & & & & \\
\hline & & $\mathrm{N} \mathrm{v}$ & $<12.96$ & & & {$[\operatorname{Ly} \alpha]$} & & & $\mathrm{N}$ v/O vI & $<-0.90$ \\
\hline & & C IV & $13.76 \pm 0.03 \pm 0.02$ & & & $13.69 \pm 0.07 \pm 0.02$ & & & C IV/O VI & $-0.14 \pm 0.08$ \\
\hline & & Si IV & $13.17 \pm 0.02 \pm 0.02$ & & & $13.12 \pm 0.03 \pm 0.02$ & & & $\mathrm{Si}$ IV/C IV & $-0.58 \pm 0.09$ \\
\hline & & $\mathrm{Fe}$ III & $13.72 \pm 0.04 \pm 0.08$ & & & & & & $\mathrm{Fe}$ III/C IV & $0.00 \pm 0.09$ \\
\hline & & S III & {$[14.08 \pm 0.04 \pm 0.15]$} & & & & & & & \\
\hline & $-30: 80$ & O VI & $14.29 \pm 0.02 \pm 0.02$ & & & {$[\operatorname{Ly} \beta]$} & & & & \\
\hline & & $\mathrm{N} \mathrm{v}$ & $13.34 \pm 0.09 \pm 0.01$ & & & [Unid. Contam.] & & & $\mathrm{N}$ v/O vI & $-0.95 \pm 0.09$ \\
\hline & & $\mathrm{C}$ IV & $14.03 \pm 0.02 \pm 0.01$ & & & $13.95 \pm 0.05 \pm 0.01$ & & & $\mathrm{C}$ IV/O VI & $-0.30 \pm 0.05$ \\
\hline & & Si IV & $13.49 \pm 0.02 \pm 0.01$ & & & $13.43 \pm 0.03 \pm 0.01$ & & & Si IV/C IV & $-0.53 \pm 0.06$ \\
\hline & & $\mathrm{Fe}$ III & $14.09 \pm 0.03 \pm 0.03$ & & & & & & $\mathrm{Fe}$ III/C IV & $0.10 \pm 0.06$ \\
\hline & & $S_{\text {III }}$ & {$[14.32 \pm 0.03 \pm 0.36]$} & & & & & & & \\
\hline \multirow[t]{4}{*}{ Mrk1095 } & $-50: 45$ & $\mathrm{O}_{\mathrm{VI}}$ & $13.83 \pm 0.07 \pm 0.04$ & $1 \pm 2$ & $43 \pm 5$ & {$\left[\mathrm{H}_{2}\right]$} & & & & \\
\hline & & $\mathrm{N} \mathrm{v}$ & $13.12 \pm 0.13 \pm 0.04$ & $4 \pm 6$ & $55 \pm 7$ & {$[\operatorname{Ly} \alpha]$} & & & $\mathrm{N}$ v/O vI & $-0.71 \pm 0.15$ \\
\hline & & $\mathrm{Fe}$ III & $14.22 \pm 0.06 \pm 0.03$ & $6 \pm 3$ & $43 \pm 5$ & & & & & \\
\hline & & $S_{\text {III }}$ & $14.81 \pm 0.05 \pm 0.04$ & & & & & & & \\
\hline \multirow[t]{24}{*}{ Mrk1383 } & $-105: 60$ & O vI & $14.58 \pm 0.02 \pm 0.01$ & $-20 \pm 2$ & $75 \pm 3$ & $14.63 \pm 0.02 \pm 0.01$ & $-23 \pm 2$ & $78 \pm 3$ & & \\
\hline & & $\mathrm{N} \mathrm{v}$ & $13.84 \pm 0.04 \pm 0.01$ & $-23 \pm 4$ & $85 \pm 5$ & {$[\operatorname{Ly} \alpha]$} & & & $\mathrm{N}$ v/O vi & $-0.77 \pm 0.05$ \\
\hline & & $\mathrm{C}$ IV & $14.40 \pm 0.04 \pm 0.01$ & $-28 \pm 1$ & $74 \pm 3$ & $14.45 \pm 0.06 \pm 0.01$ & $-29 \pm 2$ & $76 \pm 4$ & C IV/O VI & $-0.18 \pm 0.08$ \\
\hline & & Si IV & $13.81 \pm 0.04 \pm 0.01$ & $-25 \pm 1$ & $59 \pm 5$ & $13.76 \pm 0.03 \pm 0.01$ & $-24 \pm 2$ & $62 \pm 7$ & $\mathrm{Si} I V / C \mathrm{IV}$ & $-0.64 \pm 0.09$ \\
\hline & & $\mathrm{Fe}$ III & $14.34 \pm 0.02 \pm 0.02$ & $-19 \pm 2$ & $62 \pm 5$ & & & & $\mathrm{Fe}$ III/C IV & $-0.09 \pm 0.07$ \\
\hline & & $S_{\text {III }}$ & $14.58 \pm 0.03 \pm 0.03$ & $-21 \pm 2$ & $48 \pm 17$ & & & & S III/C IV & $0.15 \pm 0.08$ \\
\hline & $-105:-50$ & $\mathrm{O}_{\mathrm{VI}}$ & $13.90 \pm 0.03 \pm 0.05$ & & & $14.00 \pm 0.04 \pm 0.06$ & & & & \\
\hline & & $\mathrm{N} \mathrm{v}$ & $<13.20$ & & & $<13.40$ & & & & \\
\hline & & C IV & $13.75 \pm 0.05 \pm 0.03$ & & & $13.84 \pm 0.08 \pm 0.03$ & & & & \\
\hline & & Si IV & $12.92 \pm 0.06 \pm 0.04$ & & & $12.88 \pm 0.10 \pm 0.04$ & & & & \\
\hline & & Fe III & $<13.39$ & & & & & & & \\
\hline & & $S_{\text {III }}$ & $<13.62$ & & & & & & & \\
\hline & $-50: 10$ & O VI & $14.38 \pm 0.02 \pm 0.02$ & & & $14.41 \pm 0.02 \pm 0.03$ & & & & \\
\hline & & $\mathrm{N} \mathrm{v}$ & $13.63 \pm 0.04 \pm 0.01$ & $-22 \pm 5$ & $39 \pm 1$ & $13.73 \pm 0.07 \pm 0.02$ & & & $\mathrm{~N}$ v/O vI & $-0.71 \pm 0.09$ \\
\hline & & $\mathrm{C}$ IV & $14.24 \pm 0.05 \pm 0.01$ & $-27 \pm 4$ & $34 \pm 3$ & $14.29 \pm 0.07 \pm 0.01$ & $-28 \pm 3$ & $31 \pm 4$ & C IV/O VI & $-0.12 \pm 0.09$ \\
\hline & & Si IV & $13.73 \pm 0.05 \pm 0.01$ & $-25 \pm 2$ & $30 \pm 3$ & $13.66 \pm 0.03 \pm 0.01$ & $-25 \pm 3$ & $32 \pm 3$ & Si IV/C IV & $-0.57 \pm 0.11$ \\
\hline & & $\mathrm{Fe}$ III & $14.23 \pm 0.02 \pm 0.03$ & $-21 \pm 3$ & $28 \pm 2$ & & & & $\mathrm{Fe}$ III/C IV & $-0.04 \pm 0.09$ \\
\hline & & $S_{\text {III }}$ & $14.55 \pm 0.02 \pm 0.03$ & $-20 \pm 3$ & $29 \pm 2$ & & & & $\mathrm{~S}$ III/C IV & $0.28 \pm 0.09$ \\
\hline & $10: 60$ & O VI & $13.88 \pm 0.03 \pm 0.05$ & & & $13.93 \pm 0.04 \pm 0.07$ & & & & \\
\hline & & $\mathrm{N} \mathrm{v}$ & $<13.08$ & & & {$[\mathrm{Ly} \alpha]$} & & & $\mathrm{N}$ v/O vi & $<-0.82$ \\
\hline & & $\mathrm{C}$ IV & $13.49 \pm 0.05 \pm 0.01$ & $30 \pm 6$ & $27 \pm 5$ & $13.44 \pm 0.13 \pm 0.02$ & $41 \pm 8$ & $20 \pm 11$ & $\mathrm{C}$ IV/O VI & $-0.44 \pm 0.15$ \\
\hline & & Si IV & $12.69 \pm 0.08 \pm 0.02$ & $34 \pm 8$ & $25 \pm 8$ & $12.76 \pm 0.15 \pm 0.03$ & $34 \pm 9$ & $27 \pm 5$ & $\mathrm{Si}$ IV/C IV & $-0.74 \pm 0.22$ \\
\hline & & Fe III & $13.50 \pm 0.05 \pm 0.11$ & & & & & & & \\
\hline & & $S_{\text {III }}$ & $<13.57$ & & & & & & $\mathrm{~S}_{\mathrm{III} / \mathrm{C} \text { IV }}$ & $<0.11$ \\
\hline \multirow[t]{3}{*}{ Mrk1502 } & $-50: 40$ & O VI & $14.10 \pm 0.10 \pm 0.03$ & & & {$\left[\mathrm{H}_{2}\right]$} & & & & \\
\hline & & $\mathrm{N} \mathrm{v}$ & $<13.36$ & & & $<13.54$ & & & $\mathrm{~N}$ v/O vi & $<-0.74$ \\
\hline & & $\begin{array}{l}\text { Fe III } \\
S_{\text {III }}\end{array}$ & $\begin{array}{c}14.27 \pm 0.10 \pm 0.03 \\
{\left[\mathrm{H}_{2}\right]}\end{array}$ & $-7 \pm 3$ & $43 \pm 4$ & & & & & \\
\hline \multirow[t]{4}{*}{ Mrk1513 } & $-90: 80$ & $\mathrm{O}$ VI & $14.44 \pm 0.03 \pm 0.01$ & $0 \pm 2$ & $79 \pm 3$ & $14.51 \pm 0.03 \pm 0.02$ & $-5 \pm 8$ & $92 \pm 11$ & & \\
\hline & & $\mathrm{N} \mathrm{v}$ & $13.30 \pm 0.04 \pm 0.02$ & $4 \pm 6$ & $88 \pm 10$ & {$[\operatorname{Ly} \alpha]$} & & & $\mathrm{N}$ v/O vI & $-1.17 \pm 0.06$ \\
\hline & & $\mathrm{Fe}$ III & $14.26 \pm 0.04 \pm 0.02$ & $0 \pm 3$ & $75 \pm 7$ & & & & & \\
\hline & & $S_{\text {III }}$ & $14.63 \pm 0.05 \pm 0.25$ & & & & & & & \\
\hline \multirow[t]{4}{*}{ NGC985 } & $-55: 90$ & $\mathrm{O}_{\mathrm{VI}}$ & $14.25 \pm 0.04 \pm 0.02$ & $12 \pm 4$ & $74 \pm 6$ & $14.39 \pm 0.05 \pm 0.02$ & $20 \pm 4$ & $82 \pm 4$ & & \\
\hline & & $\mathrm{N} \mathrm{v}$ & $13.41 \pm 0.03 \pm 0.01$ & $23 \pm 3$ & $81 \pm 5$ & $13.41 \pm 0.06 \pm 0.03$ & & & $\mathrm{~N}$ v/O vI & $-0.91 \pm 0.09$ \\
\hline & & $\mathrm{Fe}$ III & $14.18 \pm 0.06 \pm 0.03$ & $-5 \pm 4$ & $64 \pm 10$ & & & & & \\
\hline & & $S_{\text {III }}$ & $14.60 \pm 0.06 \pm 0.16$ & & & & & & & \\
\hline \multirow[t]{6}{*}{ NGC1705 } & $-90: 80$ & O VI & $14.23 \pm 0.04 \pm 0.02$ & & & {$[\mathrm{CII} *]$} & & & & \\
\hline & & $\mathrm{N} \mathrm{v}$ & $13.52 \pm 0.06 \pm 0.01$ & & & [NV-NGC1705] & & & & \\
\hline & & $\mathrm{C}$ IV & $14.33 \pm 0.03 \pm 0.01$ & $-8 \pm 1$ & $71 \pm 4$ & [CIV-NGC1705] & & & & \\
\hline & & Si IV & $13.66 \pm 0.02 \pm 0.01$ & $-14 \pm 1$ & $64 \pm 5$ & $13.62 \pm 0.03 \pm 0.01$ & $-10 \pm 1$ & $60 \pm 6$ & & \\
\hline & & Fe III & $14.21 \pm 0.02 \pm 0.02$ & $-15 \pm 2$ & $66 \pm 6$ & & & & & \\
\hline & $-90: 10$ & $\begin{array}{l}\mathrm{S}_{\text {III }} \\
\mathrm{O}_{\mathrm{VI}}\end{array}$ & $\begin{array}{c}{[\text { Stellar }]} \\
14.00 \pm 0.04 \pm 0.03\end{array}$ & & & {$[\mathrm{CII} *]$} & & & & \\
\hline
\end{tabular}


Table 2

(Continuted)

\begin{tabular}{|c|c|c|c|c|c|c|c|c|c|c|}
\hline \multirow{2}{*}{$\begin{array}{l}\text { Object } \\
\text { (1) }\end{array}$} & \multirow{2}{*}{$\begin{array}{l}\text { Vel. Range } \\
\left(\mathrm{km} \mathrm{s}^{-1}\right) \\
(2)\end{array}$} & \multirow{2}{*}{$\begin{array}{l}\text { Ion } \\
\text { (3) }\end{array}$} & \multicolumn{3}{|c|}{ Strong Line } & \multicolumn{3}{|c|}{ Weak Line } & \multicolumn{2}{|c|}{ Ratios } \\
\hline & & & $\begin{array}{c}\log N \\
\left(\mathrm{~cm}^{-2}\right) \\
(4)\end{array}$ & $\begin{array}{c}\langle v\rangle \\
\left(\mathrm{km} \mathrm{s}^{-1}\right) \\
(5)\end{array}$ & $\begin{array}{c}\text { FWHM } \\
\left(\mathrm{km} \mathrm{s}^{-1}\right) \\
(6)\end{array}$ & $\begin{array}{c}\log N \\
\left(\mathrm{~cm}^{-2}\right) \\
(7)\end{array}$ & $\begin{array}{c}\langle v\rangle \\
\left(\mathrm{km} \mathrm{s}^{-1}\right) \\
(8)\end{array}$ & $\begin{array}{c}\text { FWHM } \\
\left(\mathrm{km} \mathrm{s}^{-1}\right) \\
(9)\end{array}$ & $\begin{array}{l}\text { Ions } \\
\text { (10) }\end{array}$ & $\begin{array}{c}\log \text { Ratio } \\
\text { (11) }\end{array}$ \\
\hline & & $\mathrm{N} \mathrm{v}$ & $13.28 \pm 0.07 \pm 0.01$ & & & [NV-NGC1705] & & & & \\
\hline & & C IV & $14.22 \pm 0.04 \pm 0.01$ & $-22 \pm 4$ & $44 \pm 8$ & [CIV-NGC1705] & & & & \\
\hline & & Si IV & $13.58 \pm 0.02 \pm 0.01$ & $-24 \pm 3$ & $44 \pm 6$ & $13.51 \pm 0.03 \pm 0.01$ & $-22 \pm 4$ & $37 \pm 9$ & Si IV/C IV & $-0.68 \pm 0.06$ \\
\hline & & $\begin{array}{l}\text { Fe III } \\
\text { S III }\end{array}$ & $\begin{array}{c}14.12 \pm 0.02 \pm 0.03 \\
{[\text { Stellar }]}\end{array}$ & & & & & & $\mathrm{Fe}$ III/C IV & $-0.10 \pm 0.04$ \\
\hline & $10: 80$ & O VI & $13.87 \pm 0.04 \pm 0.04$ & & & {$[\mathrm{CII} *]$} & & & & \\
\hline & & $\mathrm{N} \mathrm{v}$ & $13.17 \pm 0.07 \pm 0.02$ & & & [NV-NGC1705] & & & & \\
\hline & & C IV & $13.70 \pm 0.04 \pm 0.02$ & & & [CIV-NGC1705] & & & & \\
\hline & & Si IV & $12.96 \pm 0.04 \pm 0.02$ & & & $12.97 \pm 0.07 \pm 0.02$ & $28 \pm 6$ & $23 \pm 7$ & $\mathrm{Si}$ IV/C IV & $-0.74 \pm 0.09$ \\
\hline & & Fe III & $13.52 \pm 0.06 \pm 0.11$ & & & & & & $\mathrm{Fe}$ III/C IV & $-0.18 \pm 0.07$ \\
\hline & & S III & {$[13.97 \pm 0.05 \pm 0.09]$} & & & & & & & \\
\hline \multirow[t]{16}{*}{ NGC3516 } & $-90: 65$ & O VI & [OVI-NGC3516] & & & [OVI-NGC3516] & & & & \\
\hline & & $\mathrm{N} \mathrm{v}$ & $<13.59$ & & & $<14.12$ & & & & \\
\hline & & C IV & $13.80 \pm 0.03 \pm 0.01$ & $-36 \pm 3$ & $67 \pm 6$ & $13.91 \pm 0.04 \pm 0.01$ & $-38 \pm 4$ & $67 \pm 6$ & & \\
\hline & & Si IV & $13.23 \pm 0.10 \pm 0.01$ & $-41 \pm 7$ & $50 \pm 15$ & $<13.42$ & & & Si IV/C IV & $-0.63 \pm 0.11$ \\
\hline & & $\begin{array}{l}\text { Fe III } \\
\text { S III }\end{array}$ & $\begin{array}{c}14.25 \pm 0.09 \pm 0.03 \\
{\left[\mathrm{H}_{2}\right]}\end{array}$ & $-41 \pm 4$ & $55 \pm 17$ & & & & $\mathrm{Fe}$ III/C IV & $0.39 \pm 0.10$ \\
\hline & $-90: 0$ & O vI & $14.25 \pm 0.06 \pm 0.04$ & & & [OVI-NGC3516] & & & & \\
\hline & & $\mathrm{N} \mathrm{v}$ & $<13.45$ & & & $<13.96$ & & & $\mathrm{~N}$ v/O vI & $<-0.80$ \\
\hline & & C IV & $13.76 \pm 0.03 \pm 0.01$ & $-42 \pm 3$ & $47 \pm 2$ & $13.87 \pm 0.04 \pm 0.02$ & $-44 \pm 5$ & $53 \pm 2$ & $\mathrm{C}$ IV/O VI & $-0.44 \pm 0.08$ \\
\hline & & Si IV & $13.29 \pm 0.08 \pm 0.02$ & $-42 \pm 5$ & $44 \pm 12$ & $13.33 \pm 0.11 \pm 0.01$ & $-43 \pm 4$ & $39 \pm 10$ & Si IV/C IV & $-0.50 \pm 0.15$ \\
\hline & & $\begin{array}{l}\text { Fe III } \\
S_{\text {III }}\end{array}$ & $\begin{array}{c}14.31 \pm 0.07 \pm 0.02 \\
{\left[\mathrm{H}_{2}\right]}\end{array}$ & & & & & & $\mathrm{Fe}$ III/C IV & $0.50 \pm 0.09$ \\
\hline & $0: 65$ & O vI & [OVI-NGC3516] & & & [OVI-NGC3516] & & & & \\
\hline & & $\mathrm{N} \mathrm{v}$ & $<13.38$ & & & $<13.86$ & & & & \\
\hline & & C IV & $<12.74$ & & & $<13.06$ & & & & \\
\hline & & Si IV & $<12.83$ & & & $<13.15$ & & & & \\
\hline & & $\mathrm{Fe}$ III & $<13.73$ & & & & & & & \\
\hline & & S III & {$\left[\mathrm{H}_{2}\right]$} & & & & & & & \\
\hline \multirow[t]{18}{*}{ NGC3783 } & $-75: 100$ & O vI & {$[14.57 \pm 0.02 \pm 0.03]$} & & & {$\left[\mathrm{H}_{2}\right]$} & & & & \\
\hline & & $\mathrm{N} \mathrm{v}$ & $13.55 \pm 0.02 \pm 0.01$ & & & $13.48 \pm 0.04 \pm 0.01$ & & & & \\
\hline & & C IV & $14.33 \pm 0.02 \pm 0.01$ & & & $14.30 \pm 0.02 \pm 0.01$ & & & & \\
\hline & & Si IV & $13.78 \pm 0.02 \pm 0.01$ & & & $13.77 \pm 0.02 \pm 0.01$ & & & $\mathrm{Si} \mathrm{IV/C} \mathrm{IV}$ & $-0.53 \pm 0.04$ \\
\hline & & Fe III & {$[\mathrm{Fe} \mathrm{II}]$} & & & & & & & \\
\hline & & S III & {$\left[\mathrm{H}_{2}\right]$} & & & & & & & \\
\hline & $-75:-20$ & O VI & {$[13.78 \pm 0.02 \pm 0.04]$} & & & {$\left[\mathrm{H}_{2}\right]$} & & & & \\
\hline & & $\mathrm{N} \mathrm{v}$ & $<12.74$ & & & $<12.70$ & & & & \\
\hline & & C IV & $13.47 \pm 0.02 \pm 0.02$ & & & $13.40 \pm 0.02 \pm 0.02$ & & & & \\
\hline & & Si IV & $12.99 \pm 0.02 \pm 0.02$ & $-40 \pm 6$ & $29 \pm 7$ & $12.89 \pm 0.03 \pm 0.02$ & $-38 \pm 7$ & $28 \pm 8$ & $\mathrm{Si}$ IV/C IV & $-0.50 \pm 0.05$ \\
\hline & & $\mathrm{Fe}$ III & {$[\mathrm{Fe} \mathrm{II}]$} & & & & & & & \\
\hline & & S III & {$\left[\mathrm{H}_{2}\right]$} & & & & & & & \\
\hline & $-20: 100$ & O VI & {$[14.50 \pm 0.02 \pm 0.04]$} & & & {$\left[\mathrm{H}_{2}\right]$} & & & & \\
\hline & & $\mathrm{N} \mathrm{v}$ & $13.48 \pm 0.02 \pm 0.01$ & & & $13.48 \pm 0.04 \pm 0.01$ & & & & \\
\hline & & C IV & $14.27 \pm 0.02 \pm 0.01$ & $26 \pm 3$ & $59 \pm 3$ & $14.24 \pm 0.02 \pm 0.01$ & $24 \pm 3$ & $55 \pm 3$ & & \\
\hline & & Si IV & $13.71 \pm 0.02 \pm 0.01$ & $26 \pm 3$ & $54 \pm 4$ & $13.71 \pm 0.02 \pm 0.01$ & $26 \pm 3$ & $53 \pm 3$ & $\mathrm{Si}$ IV/C IV & $-0.55 \pm 0.04$ \\
\hline & & Fe III & {$[\mathrm{Fe} \mathrm{II}]$} & & & & & & & \\
\hline & & S III & {$\left[\mathrm{H}_{2}\right]$} & & & & & & & \\
\hline \multirow[t]{14}{*}{ NGC4151 } & $-100: 60$ & O VI & {$[14.21 \pm 0.02 \pm 0.02]$} & & & {$[14.13 \pm 0.05 \pm 0.04]$} & & & & \\
\hline & & $\mathrm{N} \mathrm{v}$ & $13.22 \pm 0.11 \pm 0.01$ & $-29 \pm 7$ & $64 \pm 17$ & [Complex continuum] & & & & \\
\hline & & C IV & $14.05 \pm 0.02 \pm 0.01$ & $-29 \pm 2$ & $72 \pm 4$ & [Complex continuum] & & & & \\
\hline & & Si IV & $13.54 \pm 0.02 \pm 0.01$ & $-26 \pm 0$ & $56 \pm 6$ & $13.53 \pm 0.03 \pm 0.01$ & $-25 \pm 2$ & $59 \pm 6$ & Si IV/C IV & $-0.51 \pm 0.04$ \\
\hline & & $\mathrm{Fe}$ III & $14.25 \pm 0.02 \pm 0.02$ & $-18 \pm 2$ & $59 \pm 6$ & & & & $\mathrm{Fe}$ III/C IV & $0.20 \pm 0.03$ \\
\hline & & S III & $14.49 \pm 0.02 \pm 0.28$ & $-21 \pm 4$ & $56 \pm 7$ & & & & $\mathrm{~S}$ III/C IV & $0.44 \pm 0.03$ \\
\hline & $-100:-60$ & O VI & $<12.74$ & & & $<13.07$ & & & & \\
\hline & & $\mathrm{N} \mathrm{v}$ & $<12.68$ & & & [Complex continuum] & & & & \\
\hline & & C IV & $13.26 \pm 0.03 \pm 0.03$ & & & [Complex continuum] & & & & \\
\hline & & Si IV & $12.40 \pm 0.07 \pm 0.07$ & & & $<12.38$ & & & & \\
\hline & & Fe III & $<12.90$ & & & & & & & \\
\hline & & S III & $13.43 \pm 0.08 \pm 0.33$ & & & & & & & \\
\hline & $-60: 25$ & O VI & {$[14.05 \pm 0.02 \pm 0.03]$} & & & {$[13.92 \pm 0.05 \pm 0.07]$} & & & & \\
\hline & & $\mathrm{N} \mathrm{v}$ & $13.19 \pm 0.08 \pm 0.01$ & $-24 \pm 4$ & $45 \pm 4$ & [Complex continuum] & & & & \\
\hline
\end{tabular}


Table 2

(Continuted)

\begin{tabular}{|c|c|c|c|c|c|c|c|c|c|c|}
\hline \multirow[t]{2}{*}{ Object } & \multirow{2}{*}{$\begin{array}{l}\text { Vel. Range } \\
\left(\mathrm{km} \mathrm{s}^{-1}\right)\end{array}$} & \multirow[t]{2}{*}{ Ion } & \multicolumn{3}{|c|}{ Strong Line } & \multicolumn{3}{|c|}{ Weak Line } & \multicolumn{2}{|c|}{ Ratios } \\
\hline & & & $\begin{array}{c}\log N \\
\left(\mathrm{~cm}^{-2}\right) \\
(4)\end{array}$ & $\begin{array}{c}\langle v\rangle \\
\left(\mathrm{km} \mathrm{s}^{-1}\right) \\
(5)\end{array}$ & $\begin{array}{c}\text { FWHM } \\
\left(\mathrm{km} \mathrm{s}^{-1}\right) \\
(6)\end{array}$ & $\begin{array}{c}\log N \\
\left(\mathrm{~cm}^{-2}\right) \\
(7)\end{array}$ & $\begin{array}{c}\langle v\rangle \\
\left(\mathrm{km} \mathrm{s}^{-1}\right) \\
(8)\end{array}$ & $\begin{array}{c}\text { FWHM } \\
\left(\mathrm{km} \mathrm{s}^{-1}\right) \\
(9)\end{array}$ & $\begin{array}{l}\text { Ions } \\
\text { (10) }\end{array}$ & $\begin{array}{c}\log \text { Ratio } \\
\text { (11) }\end{array}$ \\
\hline & & C IV & $13.97 \pm 0.02 \pm 0.01$ & $-22 \pm 4$ & $51 \pm 3$ & [Complex continuum] & & & & \\
\hline & & Si IV & $13.51 \pm 0.02 \pm 0.01$ & $-24 \pm 4$ & $49 \pm 2$ & $13.51 \pm 0.02 \pm 0.01$ & $-26 \pm 4$ & $49 \pm 2$ & Si IV/C IV & $-0.46 \pm 0.04$ \\
\hline & & $\mathrm{Fe}$ III & $14.21 \pm 0.02 \pm 0.02$ & $-18 \pm 2$ & $41 \pm 2$ & & & & $\mathrm{Fe}$ III/C IV & $0.24 \pm 0.03$ \\
\hline & & S III & $14.49 \pm 0.02 \pm 0.10$ & & & & & & & \\
\hline & $25: 60$ & O VI & {$[13.77 \pm 0.02 \pm 0.06]$} & & & {$[13.73 \pm 0.04 \pm 0.10]$} & & & & \\
\hline & & $\mathrm{N} \mathrm{v}$ & $<12.70$ & & & [Complex continuum] & & & & \\
\hline & & C IV & $<12.56$ & & & [Complex continuum] & & & & \\
\hline & & Si IV & $<12.12$ & & & $<12.40$ & & & & \\
\hline & & $\mathrm{Fe}$ III & $<12.90$ & & & & & & & \\
\hline & & S III & $<13.05$ & & & & & & & \\
\hline \multirow[t]{18}{*}{ NGC5548 } & $-110: 30$ & O VI & $14.48 \pm 0.05 \pm 0.01$ & $-31 \pm 4$ & $78 \pm 2$ & $14.31 \pm 0.09 \pm 0.03$ & & & & \\
\hline & & $\mathrm{Nv}$ & $13.63 \pm 0.03 \pm 0.01$ & $-38 \pm 3$ & $68 \pm 5$ & $13.68 \pm 0.06 \pm 0.01$ & $-51 \pm 4$ & $67 \pm 9$ & $\mathrm{~N}$ v/O vI & $-0.74 \pm 0.12$ \\
\hline & & C IV & $14.46 \pm 0.03 \pm 0.01$ & $-44 \pm 1$ & $64 \pm 3$ & $14.43 \pm 0.02 \pm 0.01$ & $-47 \pm 1$ & $63 \pm 3$ & C IV/O VI & $0.06 \pm 0.11$ \\
\hline & & Si IV & $13.81 \pm 0.02 \pm 0.01$ & $-45 \pm 1$ & $64 \pm 3$ & $13.84 \pm 0.02 \pm 0.01$ & $-43 \pm 1$ & $62 \pm 4$ & Si IV/C IV & $-0.63 \pm 0.05$ \\
\hline & & $\mathrm{Fe}$ III & $14.33 \pm 0.08 \pm 0.02$ & $-28 \pm 2$ & $52 \pm 8$ & & & & $\mathrm{Fe}$ III/C IV & $-0.12 \pm 0.09$ \\
\hline & & S III & $14.54 \pm 0.12 \pm 0.03$ & & & & & & & \\
\hline & $-110:-50$ & O VI & $13.97 \pm 0.08 \pm 0.06$ & & & $<13.95$ & & & & \\
\hline & & $\mathrm{N} \mathrm{v}$ & $13.21 \pm 0.04 \pm 0.04$ & & & $13.40 \pm 0.07 \pm 0.04$ & & & $\mathrm{~N}$ v/O vI & $-0.67 \pm 0.11$ \\
\hline & & C IV & $14.12 \pm 0.04 \pm 0.03$ & $-69 \pm 5$ & $28 \pm 7$ & $14.14 \pm 0.03 \pm 0.02$ & $-69 \pm 5$ & $28 \pm 7$ & C IV/O VI & $0.16 \pm 0.09$ \\
\hline & & Si IV & $13.49 \pm 0.03 \pm 0.03$ & $-68 \pm 5$ & $30 \pm 9$ & $13.48 \pm 0.03 \pm 0.04$ & $-68 \pm 6$ & $30 \pm 10$ & Si IV/C IV & $-0.65 \pm 0.06$ \\
\hline & & Fe III & $<13.81$ & & & & & & $\mathrm{Fe}$ III/C IV & $<-0.32$ \\
\hline & & S III & $<14.28$ & & & & & & $\mathrm{~S}$ III/C IV & $<0.15$ \\
\hline & $-50: 30$ & O VI & $14.33 \pm 0.05 \pm 0.03$ & & & $14.15 \pm 0.10 \pm 0.06$ & & & & \\
\hline & & $\mathrm{N} \mathrm{v}$ & $13.43 \pm 0.03 \pm 0.03$ & & & $13.39 \pm 0.08 \pm 0.04$ & & & $\mathrm{~N}$ v/O vI & $-0.83 \pm 0.14$ \\
\hline & & C IV & $14.21 \pm 0.03 \pm 0.02$ & $-25 \pm 6$ & $43 \pm 3$ & $14.14 \pm 0.03 \pm 0.02$ & $-25 \pm 6$ & $39 \pm 5$ & C IV/O VI & $-0.06 \pm 0.12$ \\
\hline & & Si IV & $13.54 \pm 0.03 \pm 0.03$ & $-24 \pm 6$ & $43 \pm 3$ & $13.61 \pm 0.03 \pm 0.03$ & $-25 \pm 6$ & $41 \pm 4$ & $\mathrm{Si}$ IV/C IV & $-0.61 \pm 0.06$ \\
\hline & & $\mathrm{Fe}$ III & $14.25 \pm 0.08 \pm 0.04$ & & & & & & $\mathrm{Fe}$ III/C IV & $0.07 \pm 0.09$ \\
\hline & & S III & $14.35 \pm 0.13 \pm 0.06$ & & & & & & & \\
\hline \multirow[t]{18}{*}{ NGC7469 } & $-75: 65$ & O VI & {$[14.01 \pm 0.04 \pm 0.04]$} & & & {$\left[\mathrm{H}_{2}\right]$} & & & & \\
\hline & & $\mathrm{N} \mathrm{v}$ & $<12.69$ & & & $<13.24$ & & & & \\
\hline & & C IV & $14.07 \pm 0.02 \pm 0.01$ & $1 \pm 2$ & $79 \pm 2$ & $14.08 \pm 0.03 \pm 0.01$ & $-3 \pm 3$ & $84 \pm 2$ & & \\
\hline & & Si IV & $13.56 \pm 0.02 \pm 0.01$ & $-4 \pm 1$ & $68 \pm 3$ & $13.51 \pm 0.04 \pm 0.01$ & $-3 \pm 3$ & $71 \pm 5$ & $\mathrm{Si} \mathrm{IV/C} \mathrm{IV}$ & $-0.53 \pm 0.06$ \\
\hline & & Fe III & $14.24 \pm 0.04 \pm 0.02$ & & & & & & $\mathrm{Fe}$ III/C IV & $0.17 \pm 0.06$ \\
\hline & & S III & {$\left[\mathrm{H}_{2}\right]$} & & & & & & & \\
\hline & $-75:-10$ & O vI & {$[13.78 \pm 0.05 \pm 0.06]$} & & & {$\left[\mathrm{H}_{2}\right]$} & & & & \\
\hline & & $\mathrm{N} \mathrm{v}$ & $12.48 \pm 0.15 \pm 0.04$ & & & $<13.04$ & & & & \\
\hline & & C IV & $13.66 \pm 0.03 \pm 0.01$ & & & $13.70 \pm 0.05 \pm 0.02$ & & & & \\
\hline & & Si IV & $13.24 \pm 0.03 \pm 0.01$ & & & $13.18 \pm 0.05 \pm 0.01$ & $-31 \pm 5$ & $28 \pm 7$ & Si IV/C IV & $-0.47 \pm 0.08$ \\
\hline & & Fe III & $14.03 \pm 0.04 \pm 0.05$ & & & & & & $\mathrm{Fe}$ III/C IV & $0.35 \pm 0.07$ \\
\hline & & $\mathrm{S}_{\text {III }}$ & {$\left[\mathrm{H}_{2}\right]$} & & & & & & & \\
\hline & $-10: 65$ & O VI & {$[13.67 \pm 0.07 \pm 0.08]$} & & & {$\left[\mathrm{H}_{2}\right]$} & & & & \\
\hline & & $\mathrm{N} \mathrm{v}$ & $<12.61$ & & & $<13.08$ & & & & \\
\hline & & C IV & $13.86 \pm 0.02 \pm 0.01$ & & & $13.86 \pm 0.04 \pm 0.01$ & & & & \\
\hline & & Si IV & $13.29 \pm 0.03 \pm 0.01$ & & & $13.25 \pm 0.05 \pm 0.01$ & & & $\mathrm{Si}$ IV/C IV & $-0.59 \pm 0.07$ \\
\hline & & $\mathrm{Fe}$ III & $13.88 \pm 0.06 \pm 0.06$ & & & & & & $\mathrm{Fe}$ III/C IV & $0.02 \pm 0.07$ \\
\hline & & S III & {$\left[\mathrm{H}_{2}\right]$} & & & & & & & \\
\hline \multirow[t]{4}{*}{ PG0804+761 } & $-90: 60$ & O vI & $14.49 \pm 0.02 \pm 0.01$ & & & {$\left[\mathrm{H}_{2}\right]$} & & & & \\
\hline & & $\mathrm{N} \mathrm{v}$ & {$[\operatorname{Ly} \alpha]$} & & & $<13.39$ & & & $\mathrm{~N}$ v/O vi & $<-1.10$ \\
\hline & & $\mathrm{Fe}$ III & $14.28 \pm 0.02 \pm 0.01$ & $-33 \pm 1$ & $51 \pm 5$ & & & & & \\
\hline & & S III & $14.78 \pm 0.02 \pm 0.21$ & & & & & & & \\
\hline \multirow[t]{13}{*}{ PG0953+414 } & $-125: 70$ & O VI & $14.36 \pm 0.02 \pm 0.01$ & $-14 \pm 5$ & $106 \pm 4$ & $14.31 \pm 0.04 \pm 0.05$ & $-20 \pm 7$ & $113 \pm 12$ & & \\
\hline & & $\mathrm{N} \mathrm{v}$ & $<13.22$ & & & $<13.55$ & & & $\mathrm{~N}$ v/O vI & $<-1.12$ \\
\hline & & C IV & $13.97 \pm 0.03 \pm 0.01$ & $-22 \pm 3$ & $114 \pm 3$ & $13.84 \pm 0.08 \pm 0.01$ & $-21 \pm 6$ & $116 \pm 5$ & $\mathrm{C}$ IV/O VI & $-0.44 \pm 0.10$ \\
\hline & & Si IV & $13.34 \pm 0.05 \pm 0.01$ & & & $13.32 \pm 0.07 \pm 0.01$ & $-20 \pm 3$ & $104 \pm 5$ & & \\
\hline & & Fe III & $14.14 \pm 0.04 \pm 0.03$ & & & & & & & \\
\hline & & S III & $14.27 \pm 0.07 \pm 0.12$ & & & & & & & \\
\hline & $-125:-35$ & $\mathrm{O}$ VI & $13.88 \pm 0.03 \pm 0.04$ & & & $13.86 \pm 0.07 \pm 0.13$ & & & & \\
\hline & & $\mathrm{N} \mathrm{v}$ & $<13.03$ & & & $<13.36$ & & & $\mathrm{~N}$ v/O vi & $<-0.84$ \\
\hline & & C IV & $13.56 \pm 0.04 \pm 0.01$ & & & $13.42 \pm 0.12 \pm 0.01$ & & & $\mathrm{C}$ IV/O VI & $-0.38 \pm 0.15$ \\
\hline & & Si IV & {$[13.09 \pm 0.05 \pm 0.01]$} & & & $12.88 \pm 0.10 \pm 0.01$ & & & Si IV/C IV & $-0.61 \pm 0.16$ \\
\hline & & $\mathrm{Fe}$ III & $13.90 \pm 0.04 \pm 0.05$ & & & & & & & \\
\hline & & $\mathrm{S}_{\text {III }}$ & $14.06 \pm 0.08 \pm 0.08$ & & & & & & & \\
\hline & $-35: 70$ & $\mathrm{O}$ VI & $14.19 \pm 0.02 \pm 0.02$ & & & $14.13 \pm 0.05 \pm 0.04$ & & & & \\
\hline
\end{tabular}


Table 2

(Continuted)

\begin{tabular}{|c|c|c|c|c|c|c|c|c|c|c|}
\hline \multirow{2}{*}{$\begin{array}{l}\text { Object } \\
\text { (1) }\end{array}$} & \multirow{2}{*}{$\begin{array}{l}\text { Vel. Range } \\
\left(\mathrm{km} \mathrm{s}^{-1}\right) \\
(2)\end{array}$} & \multirow{2}{*}{$\begin{array}{l}\text { Ion } \\
\text { (3) }\end{array}$} & \multicolumn{3}{|c|}{ Strong Line } & \multicolumn{3}{|c|}{ Weak Line } & \multicolumn{2}{|c|}{ Ratios } \\
\hline & & & $\begin{array}{c}\log N \\
\left(\mathrm{~cm}^{-2}\right) \\
(4)\end{array}$ & $\begin{array}{c}\langle v\rangle \\
\left(\mathrm{km} \mathrm{s}^{-1}\right) \\
(5)\end{array}$ & $\begin{array}{c}\text { FWHM } \\
\left(\mathrm{km} \mathrm{s}^{-1}\right) \\
(6)\end{array}$ & $\begin{array}{c}\log N \\
\left(\mathrm{~cm}^{-2}\right) \\
(7)\end{array}$ & $\begin{array}{c}\langle v\rangle \\
\left(\mathrm{km} \mathrm{s}^{-1}\right) \\
(8)\end{array}$ & $\begin{array}{c}\text { FWHM } \\
\left(\mathrm{km} \mathrm{s}^{-1}\right) \\
(9)\end{array}$ & $\begin{array}{l}\text { Ions } \\
\text { (10) }\end{array}$ & $\begin{array}{c}\log \text { Ratio } \\
\text { (11) }\end{array}$ \\
\hline \multirow{30}{*}{$\begin{array}{l}\text { PG1049-005 } \\
\text { PG1116+215 }\end{array}$} & & $\mathrm{N} \mathrm{v}$ & $<13.09$ & & & $<13.40$ & & & $\mathrm{~N}$ v/O vI & $<-1.07$ \\
\hline & & C IV & $13.76 \pm 0.03 \pm 0.01$ & & & $13.63 \pm 0.08 \pm 0.01$ & & & $\mathrm{C}$ IV/O vI & $-0.46 \pm 0.10$ \\
\hline & & Si IV & $13.00 \pm 0.07 \pm 0.01$ & & & $13.14 \pm 0.07 \pm 0.01$ & & & Si IV/C IV & $-0.63 \pm 0.13$ \\
\hline & & $\mathrm{Fe}$ III & $13.79 \pm 0.06 \pm 0.06$ & & & & & & & \\
\hline & & $\mathrm{S}_{\text {III }}$ & $13.89 \pm 0.11 \pm 0.22$ & & & & & & & \\
\hline & $-60: 40$ & $\mathrm{~N} \mathrm{v}$ & $13.67 \pm 0.06 \pm 0.02$ & $-4 \pm 4$ & $46 \pm 7$ & $<13.63$ & & & & \\
\hline & $-85: 85$ & O VI & $14.21 \pm 0.02 \pm 0.01$ & $13 \pm 2$ & $80 \pm 4$ & $14.26 \pm 0.03 \pm 0.02$ & $12 \pm 4$ & $82 \pm 6$ & & \\
\hline & & $\mathrm{N} \mathrm{v}$ & $<13.33$ & & & $<13.45$ & & & & \\
\hline & & C IV & $14.20 \pm 0.02 \pm 0.01$ & & & $14.15 \pm 0.03 \pm 0.01$ & & & & \\
\hline & & Si IV & $13.67 \pm 0.02 \pm 0.01$ & $-18 \pm 2$ & $78 \pm 3$ & $13.70 \pm 0.02 \pm 0.01$ & $-10 \pm 2$ & $82 \pm 4$ & & \\
\hline & & $\mathrm{Fe}$ III & $14.37 \pm 0.02 \pm 0.01$ & $-21 \pm 2$ & $72 \pm 5$ & & & & & \\
\hline & & S III & $14.60 \pm 0.03 \pm 0.07$ & $-14 \pm 2$ & $71 \pm 5$ & & & & & \\
\hline & $-85:-20$ & O VI & $13.51 \pm 0.05 \pm 0.04$ & & & $13.50 \pm 0.10 \pm 0.06$ & & & & \\
\hline & & $\mathrm{N} \mathrm{v}$ & $<12.94$ & & & $<13.27$ & & & $\mathrm{~N}$ v/O vI & $<-0.57$ \\
\hline & & C IV & $13.86 \pm 0.02 \pm 0.01$ & & & $13.84 \pm 0.04 \pm 0.01$ & & & $\mathrm{C}$ IV/O vi & $0.34 \pm 0.12$ \\
\hline & & Si IV & $13.38 \pm 0.02 \pm 0.01$ & & & $13.32 \pm 0.02 \pm 0.01$ & & & $\mathrm{Si}$ IV/C IV & $-0.50 \pm 0.05$ \\
\hline & & $\mathrm{Fe}$ III & $14.12 \pm 0.03 \pm 0.02$ & & & & & & $\mathrm{Fe}$ III/C IV & $0.27 \pm 0.05$ \\
\hline & & $S_{\text {III }}$ & $14.25 \pm 0.04 \pm 0.03$ & & & & & & S III/C IV & $0.40 \pm 0.06$ \\
\hline & $-20: 30$ & $\mathrm{O}$ VI & $13.90 \pm 0.03 \pm 0.03$ & & & $13.97 \pm 0.04 \pm 0.03$ & & & & \\
\hline & & $\mathrm{N} \mathrm{v}$ & $<12.91$ & & & $<13.17$ & & & $\mathrm{~N}$ v/O vi & $<-1.03$ \\
\hline & & C IV & $13.78 \pm 0.03 \pm 0.02$ & & & $13.73 \pm 0.05 \pm 0.01$ & $4 \pm 5$ & $27 \pm 2$ & $\mathrm{C}$ IV/O VI & $-0.18 \pm 0.08$ \\
\hline & & Si IV & $13.30 \pm 0.02 \pm 0.02$ & $0 \pm 6$ & $29 \pm 2$ & $13.38 \pm 0.02 \pm 0.02$ & $3 \pm 6$ & $31 \pm 2$ & Si IV/C IV & $-0.42 \pm 0.07$ \\
\hline & & $\mathrm{Fe}$ III & $13.96 \pm 0.03 \pm 0.03$ & & & & & & $\mathrm{Fe}$ III/C IV & $0.20 \pm 0.07$ \\
\hline & & $S_{\text {III }}$ & $14.29 \pm 0.03 \pm 0.06$ & & & & & & S III/C IV & $0.53 \pm 0.07$ \\
\hline & $30: 85$ & O VI & $13.76 \pm 0.03 \pm 0.03$ & & & $13.81 \pm 0.05 \pm 0.05$ & & & & \\
\hline & & $\mathrm{N} \mathrm{v}$ & $<12.96$ & & & $<13.22$ & & & $\mathrm{~N}$ v/O vi & $<-0.83$ \\
\hline & & C IV & $13.47 \pm 0.04 \pm 0.02$ & & & $13.30 \pm 0.10 \pm 0.03$ & & & $\mathrm{C}$ IV/O VI & $-0.41 \pm 0.13$ \\
\hline & & Si IV & $12.59 \pm 0.06 \pm 0.03$ & & & $12.79 \pm 0.08 \pm 0.03$ & & & $\mathrm{Si}$ IV/C IV & $-0.69 \pm 0.15$ \\
\hline & & $\mathrm{Fe}$ III & $13.26 \pm 0.12 \pm 0.06$ & & & & & & $\mathrm{Fe}$ III/C IV & $-0.12 \pm 0.16$ \\
\hline & & S III & $<13.54$ & & & & & & $\mathrm{~S}$ III/C IV & $<0.16$ \\
\hline \multirow[t]{6}{*}{ PG1211+143 } & $-80: 60$ & O VI & $14.16 \pm 0.03 \pm 0.02$ & $6 \pm 3$ & $66 \pm 4$ & $14.20 \pm 0.05 \pm 0.06$ & $3 \pm 4$ & $74 \pm 5$ & & \\
\hline & $-60: 60$ & $\mathrm{~N} \mathrm{v}$ & $13.34 \pm 0.05 \pm 0.01$ & $-7 \pm 5$ & $66 \pm 5$ & {$[\operatorname{Ly} \alpha]$} & & & $\mathrm{N}$ v/O vi & $-0.84 \pm 0.08$ \\
\hline & $-80: 60$ & C IV & $14.09 \pm 0.02 \pm 0.01$ & $-9 \pm 1$ & $53 \pm 5$ & $14.09 \pm 0.02 \pm 0.01$ & $-4 \pm 2$ & $62 \pm 4$ & $\mathrm{C}$ IV/O VI & $-0.09 \pm 0.07$ \\
\hline & & Si IV & $13.61 \pm 0.02 \pm 0.01$ & $-6 \pm 1$ & $51 \pm 5$ & {$[13.72 \pm 0.02 \pm 0.01]$} & & & Si IV/C IV & $-0.48 \pm 0.04$ \\
\hline & & $\mathrm{Fe}$ III & $14.18 \pm 0.03 \pm 0.02$ & $-6 \pm 2$ & $56 \pm 4$ & & & & $\mathrm{Fe}$ III/C IV & $0.09 \pm 0.04$ \\
\hline & & S III & $14.51 \pm 0.04 \pm 0.18$ & & & & & & $\mathrm{~S}$ III/C IV & $0.42 \pm 0.05$ \\
\hline \multirow[t]{6}{*}{ PG1216+069 } & $-60: 60$ & O VI & {$[\operatorname{Ly} \beta]$} & & & [Unid. Contam.] & & & & \\
\hline & & $\mathrm{N} \mathrm{v}$ & [CIII] & & & {$[\operatorname{Ly} \beta]$} & & & & \\
\hline & & $\mathrm{C}$ IV & [Unid. Contam.] & & & [Unid. Contam.] & & & & \\
\hline & & Si IV & $13.57 \pm 0.03 \pm 0.01$ & $-1 \pm 2$ & $49 \pm 4$ & $13.46 \pm 0.05 \pm 0.01$ & $0 \pm 3$ & $31 \pm 9$ & & \\
\hline & & Fe III & $<14.26$ & & & & & & & \\
\hline & & S III & $<14.74$ & & & & & & & \\
\hline \multirow[t]{18}{*}{ PG1259+593 } & $-80: 50$ & $\mathrm{O}_{\mathrm{VI}}$ & $14.11 \pm 0.02 \pm 0.01$ & $-6 \pm 5$ & $79 \pm 2$ & $14.15 \pm 0.04 \pm 0.02$ & $-12 \pm 6$ & $81 \pm 4$ & & \\
\hline & & $\mathrm{N} \mathrm{v}$ & $13.14 \pm 0.12 \pm 0.01$ & & & {$[\operatorname{Ly} \alpha]$} & & & $\mathrm{N}$ v/O vi & $-0.99 \pm 0.13$ \\
\hline & & C IV & $13.75 \pm 0.04 \pm 0.01$ & $-5 \pm 3$ & $65 \pm 4$ & $13.76 \pm 0.08 \pm 0.01$ & $-15 \pm 7$ & $84 \pm 7$ & $\mathrm{C}$ IV/O VI & $-0.37 \pm 0.10$ \\
\hline & & Si IV & $13.33 \pm 0.02 \pm 0.01$ & $-14 \pm 3$ & $67 \pm 6$ & $13.39 \pm 0.06 \pm 0.01$ & $-12 \pm 3$ & $77 \pm 4$ & Si IV/C IV & $-0.40 \pm 0.11$ \\
\hline & & $\mathrm{Fe}$ III & $14.11 \pm 0.02 \pm 0.01$ & $-18 \pm 3$ & $71 \pm 3$ & & & & $\mathrm{Fe}$ III/C IV & $0.35 \pm 0.09$ \\
\hline & & S III & $14.32 \pm 0.04 \pm 0.04$ & & & & & & & \\
\hline & $-80:-27$ & $\mathrm{O}$ VI & $13.59 \pm 0.04 \pm 0.04$ & & & $13.71 \pm 0.06 \pm 0.04$ & & & & \\
\hline & & $\mathrm{N} \mathrm{v}$ & $12.98 \pm 0.11 \pm 0.02$ & & & {$[\operatorname{Ly} \alpha]$} & & & & \\
\hline & & C IV & $13.16 \pm 0.07 \pm 0.03$ & & & $13.36 \pm 0.11 \pm 0.03$ & & & C IV/O VI & $-0.39 \pm 0.15$ \\
\hline & & Si IV & $12.88 \pm 0.04 \pm 0.02$ & & & $12.99 \pm 0.07 \pm 0.01$ & & & $\mathrm{Si}$ IV/C IV & $-0.32 \pm 0.15$ \\
\hline & & Fe III & $13.74 \pm 0.03 \pm 0.03$ & & & & & & $\mathrm{Fe}$ III/C IV & $0.48 \pm 0.13$ \\
\hline & & S III & $14.02 \pm 0.05 \pm 0.03$ & & & & & & $\mathrm{~S}$ III/C IV & $0.76 \pm 0.14$ \\
\hline & $-27: 50$ & O VI & $13.97 \pm 0.02 \pm 0.01$ & & & $13.97 \pm 0.05 \pm 0.02$ & & & & \\
\hline & & $\mathrm{N} \mathrm{v}$ & $<13.00$ & & & {$[\operatorname{Ly} \alpha]$} & & & $\mathrm{N}$ v/O vI & $<-0.97$ \\
\hline & & C IV & $13.64 \pm 0.04 \pm 0.01$ & & & $13.55 \pm 0.09 \pm 0.01$ & & & $\mathrm{C}$ IV/O VI & $-0.37 \pm 0.11$ \\
\hline & & Si IV & $13.15 \pm 0.03 \pm 0.01$ & & & $13.18 \pm 0.07 \pm 0.01$ & & & Si IV/C IV & $-0.44 \pm 0.13$ \\
\hline & & Fe III & $13.88 \pm 0.03 \pm 0.02$ & & & & & & $\mathrm{Fe}$ III/C IV & $0.28 \pm 0.10$ \\
\hline & & S III & $14.04 \pm 0.05 \pm 0.08$ & & & & & & & \\
\hline \multirow[t]{4}{*}{ PG1302-102 } & $-50: 60$ & $\mathrm{O}$ VI & $14.22 \pm 0.03 \pm 0.02$ & $1 \pm 2$ & $52 \pm 3$ & $14.33 \pm 0.04 \pm 0.02$ & $-1 \pm 3$ & $60 \pm 3$ & & \\
\hline & & $\mathrm{N} \mathrm{v}$ & {$[\operatorname{Ly} \alpha]$} & & & $<13.87$ & & & & \\
\hline & & C IV & $14.17 \pm 0.05 \pm 0.01$ & $7 \pm 1$ & $50 \pm 5$ & $14.20 \pm 0.04 \pm 0.01$ & $8 \pm 2$ & $48 \pm 5$ & & \\
\hline & & & & & 20 & & & & & \\
\hline
\end{tabular}


Table 2

(Continuted)

\begin{tabular}{|c|c|c|c|c|c|c|c|c|c|c|}
\hline \multirow[t]{2}{*}{ Object } & \multirow{2}{*}{$\begin{array}{l}\text { Vel. Range } \\
\left(\mathrm{km} \mathrm{s}^{-1}\right) \\
(2)\end{array}$} & \multirow{2}{*}{$\begin{array}{l}\text { Ion } \\
\text { (3) }\end{array}$} & \multicolumn{3}{|c|}{ Strong Line } & \multicolumn{3}{|c|}{ Weak Line } & \multicolumn{2}{|c|}{ Ratios } \\
\hline & & & $\begin{array}{c}\log N \\
\left(\mathrm{~cm}^{-2}\right) \\
(4)\end{array}$ & $\begin{array}{c}\langle v\rangle \\
\left(\mathrm{km} \mathrm{s}^{-1}\right) \\
(5)\end{array}$ & $\begin{array}{c}\text { FWHM } \\
\left(\mathrm{km} \mathrm{s}^{-1}\right) \\
(6)\end{array}$ & $\begin{array}{c}\log N \\
\left(\mathrm{~cm}^{-2}\right) \\
(7)\end{array}$ & $\begin{array}{c}\langle v\rangle \\
\left(\mathrm{km} \mathrm{s}^{-1}\right) \\
(8)\end{array}$ & $\begin{array}{c}\text { FWHM } \\
\left(\mathrm{km} \mathrm{s}^{-1}\right) \\
(9)\end{array}$ & $\begin{array}{l}\text { Ions } \\
\text { (10) }\end{array}$ & $\begin{array}{c}\log \text { Ratio } \\
\text { (11) }\end{array}$ \\
\hline \multirow{15}{*}{ (1) } & & Si IV & $13.73 \pm 0.04 \pm 0.01$ & $6 \pm 2$ & $56 \pm 3$ & $13.62 \pm 0.07 \pm 0.01$ & $7 \pm 2$ & $45 \pm 7$ & & \\
\hline & & Fe III & $14.12 \pm 0.04 \pm 0.23$ & $10 \pm 28$ & $39 \pm 16$ & & & & & \\
\hline & & $\mathrm{S}_{\text {III }}$ & $14.53 \pm 0.05 \pm 0.09$ & & & & & & & \\
\hline & $-50: 0$ & O VI & $13.92 \pm 0.04 \pm 0.04$ & & & $14.09 \pm 0.05 \pm 0.05$ & & & & \\
\hline & & $\mathrm{N} \mathrm{v}$ & {$[\operatorname{Ly} \alpha]$} & & & $<13.68$ & & & $\mathrm{~N}$ v/O vI & $<-0.33$ \\
\hline & & C IV & $13.64 \pm 0.05 \pm 0.03$ & & & $13.68 \pm 0.07 \pm 0.04$ & & & C IV/O VI & $-0.35 \pm 0.11$ \\
\hline & & Si IV & $13.30 \pm 0.05 \pm 0.03$ & & & $13.13 \pm 0.11 \pm 0.03$ & & & Si IV/C IV & $-0.45 \pm 0.15$ \\
\hline & & Fe III & $13.61 \pm 0.08 \pm 0.23$ & & & & & & $\mathrm{Fe}$ III/C IV & $-0.05 \pm 0.12$ \\
\hline & & S III & $14.13 \pm 0.07 \pm 0.08$ & & & & & & $\mathrm{~S}$ III/C IV & $0.47 \pm 0.11$ \\
\hline & $0: 60$ & O VI & $13.94 \pm 0.04 \pm 0.04$ & & & $13.99 \pm 0.06 \pm 0.06$ & & & & \\
\hline & & $\mathrm{N} \mathrm{v}$ & $<13.44$ & & & $<13.67$ & & & $\mathrm{~N}$ v/O vi & $<-0.52$ \\
\hline & & C IV & $14.03 \pm 0.06 \pm 0.01$ & $19 \pm 4$ & $29 \pm 5$ & $14.06 \pm 0.04 \pm 0.01$ & $18 \pm 5$ & $33 \pm 3$ & C IV/O VI & $0.08 \pm 0.10$ \\
\hline & & Si IV & $13.55 \pm 0.05 \pm 0.02$ & $20 \pm 6$ & $37 \pm 2$ & $13.48 \pm 0.09 \pm 0.01$ & $17 \pm 5$ & $26 \pm 6$ & $\mathrm{Si}$ IV/C IV & $-0.52 \pm 0.12$ \\
\hline & & Fe III & $13.98 \pm 0.05 \pm 0.24$ & & & & & & $\mathrm{Fe}$ III/C IV & $-0.06 \pm 0.09$ \\
\hline & & $\mathrm{S}_{\text {IIII }}$ & $14.34 \pm 0.05 \pm 0.14$ & & & & & & $\mathrm{~S}$ III/C IV & $0.30 \pm 0.09$ \\
\hline \multirow[t]{4}{*}{ PG1351+640 } & $-60: 70$ & O VI & $14.24 \pm 0.03 \pm 0.02$ & $-1 \pm 6$ & $74 \pm 4$ & $14.37 \pm 0.06 \pm 0.03$ & & & & \\
\hline & & $\mathrm{N} \mathrm{v}$ & $13.24 \pm 0.08 \pm 0.02$ & & & $<13.30$ & & & $\mathrm{~N}$ v/O vI & $-1.06 \pm 0.11$ \\
\hline & & Fe III & [Unid. Contam.] & & & & & & & \\
\hline & & S III & {$\left[\mathrm{H}_{2}\right]$} & & & & & & & \\
\hline \multirow[t]{12}{*}{ PG1444+407 } & $-115: 20$ & O VI & $14.35 \pm 0.11 \pm 0.02$ & & & $14.51 \pm 0.13 \pm 0.05$ & & & & \\
\hline & & $\mathrm{N} \mathrm{v}$ & {$[\operatorname{Ly} \alpha]$} & & & $13.65 \pm 0.10 \pm 0.01$ & & & $\mathrm{~N}$ v/O vI & $-0.78 \pm 0.20$ \\
\hline & & C IV & $14.09 \pm 0.02 \pm 0.01$ & $-49 \pm 1$ & $50 \pm 5$ & $14.22 \pm 0.04 \pm 0.01$ & $-47 \pm 2$ & $58 \pm 4$ & $\mathrm{C}$ IV/O VI & $-0.28 \pm 0.17$ \\
\hline & & Si IV & $13.49 \pm 0.03 \pm 0.01$ & $-43 \pm 1$ & $59 \pm 4$ & {$[\operatorname{Ly} \alpha]$} & & & $\mathrm{Si}$ IV/C IV & $-0.66 \pm 0.05$ \\
\hline & $-115: 80$ & Fe III & $14.42 \pm 0.12 \pm 0.02$ & & & & & & & \\
\hline & & $S_{\text {III }}$ & $<14.90$ & & & & & & $\mathrm{~S}$ III/C IV & $<0.75$ \\
\hline & $-115: 20$ & O vI & $14.35 \pm 0.11 \pm 0.02$ & & & $14.51 \pm 0.13 \pm 0.05$ & & & & \\
\hline & & $\mathrm{N} \mathrm{v}$ & {$[\operatorname{Ly} \alpha]$} & & & $13.65 \pm 0.10 \pm 0.01$ & & & $\mathrm{~N}$ v/O vI & $-0.78 \pm 0.20$ \\
\hline & & C IV & $14.09 \pm 0.02 \pm 0.01$ & $-49 \pm 1$ & $50 \pm 5$ & $14.22 \pm 0.04 \pm 0.01$ & $-47 \pm 2$ & $58 \pm 4$ & $\mathrm{C}$ IV/O VI & $-0.28 \pm 0.17$ \\
\hline & & Si IV & $13.49 \pm 0.03 \pm 0.01$ & $-43 \pm 1$ & $59 \pm 4$ & {$[\operatorname{Ly} \alpha]$} & & & $\mathrm{Si}$ IV/C IV & $-0.66 \pm 0.05$ \\
\hline & & Fe III & $<14.27$ & & & & & & $\mathrm{Fe}$ III/C IV & $<0.12$ \\
\hline & & S III & $<14.81$ & & & & & & S III/C IV & $<0.66$ \\
\hline \multirow[t]{3}{*}{ PG1718+481 } & $-65: 50$ & C IV & $14.09 \pm 0.02 \pm 0.01$ & $-16 \pm 3$ & $57 \pm 4$ & $14.12 \pm 0.03 \pm 0.01$ & $-14 \pm 4$ & $61 \pm 3$ & & \\
\hline & $-65:-15$ & $\mathrm{C}$ IV & $13.82 \pm 0.03 \pm 0.04$ & $-36 \pm 6$ & $27 \pm 3$ & $13.83 \pm 0.04 \pm 0.04$ & $-37 \pm 6$ & $26 \pm 2$ & & \\
\hline & $-15: 50$ & $\mathrm{C}$ IV & $13.79 \pm 0.03 \pm 0.03$ & $6 \pm 6$ & $31 \pm 4$ & $13.82 \pm 0.04 \pm 0.03$ & $9 \pm 6$ & $31 \pm 4$ & & \\
\hline \multirow[t]{15}{*}{ PHL1811 } & $-65: 80$ & O VI & $14.35 \pm 0.02 \pm 0.01$ & $11 \pm 4$ & $75 \pm 3$ & $14.27 \pm 0.04 \pm 0.16$ & $16 \pm 5$ & $72 \pm 16$ & & \\
\hline & & $\mathrm{N} \mathrm{v}$ & $13.36 \pm 0.08 \pm 0.01$ & & & $<13.35$ & & & & \\
\hline & & C IV & $14.29 \pm 0.02 \pm 0.01$ & $8 \pm 1$ & $74 \pm 2$ & $14.32 \pm 0.03 \pm 0.01$ & $7 \pm 2$ & $78 \pm 2$ & & \\
\hline & & Si IV & $13.61 \pm 0.02 \pm 0.01$ & $9 \pm 1$ & $69 \pm 3$ & $13.55 \pm 0.03 \pm 0.01$ & $7 \pm 2$ & $66 \pm 4$ & & \\
\hline & & $\begin{array}{l}\text { Fe III } \\
\text { S III }\end{array}$ & $\begin{array}{c}14.18 \pm 0.04 \pm 0.02 \\
{\left[\mathrm{H}_{2}\right]}\end{array}$ & $6 \pm 2$ & $63 \pm 6$ & & & & & \\
\hline & $-65: 30$ & O VI & $14.20 \pm 0.03 \pm 0.02$ & $-6 \pm 5$ & $49 \pm 2$ & $14.10 \pm 0.05 \pm 0.17$ & & & & \\
\hline & & $\mathrm{N} \mathrm{v}$ & $13.22 \pm 0.08 \pm 0.01$ & $-13 \pm 3$ & $47 \pm 7$ & $<13.25$ & & & $\mathrm{~N}$ v/O vI & $-0.93 \pm 0.10$ \\
\hline & & $\mathrm{C}$ IV & $14.16 \pm 0.02 \pm 0.01$ & $-8 \pm 3$ & $45 \pm 5$ & $14.18 \pm 0.03 \pm 0.01$ & $-10 \pm 4$ & $49 \pm 3$ & $\mathrm{C}$ IV/O VI & $0.02 \pm 0.07$ \\
\hline & & Si IV & $13.50 \pm 0.02 \pm 0.01$ & $-5 \pm 3$ & $42 \pm 5$ & $13.45 \pm 0.03 \pm 0.01$ & $-4 \pm 4$ & $42 \pm 9$ & $\mathrm{Si} \mathrm{IV/C} \mathrm{IV}$ & $-0.70 \pm 0.06$ \\
\hline & & $\begin{array}{l}\mathrm{Fe} \text { III } \\
\mathrm{S}_{\text {III }}\end{array}$ & $\begin{array}{c}14.07 \pm 0.04 \pm 0.03 \\
{\left[\mathrm{H}_{2}\right]}\end{array}$ & $-5 \pm 5$ & $39 \pm 8$ & & & & $\mathrm{Fe}$ III/C IV & $-0.10 \pm 0.06$ \\
\hline & $30: 80$ & O VI & $13.84 \pm 0.04 \pm 0.05$ & $51 \pm 6$ & $30 \pm 2$ & $13.80 \pm 0.07 \pm 0.16$ & & & & \\
\hline & & $\mathrm{N} \mathrm{v}$ & $12.80 \pm 0.15 \pm 0.02$ & $58 \pm 5$ & $28 \pm 5$ & $<13.07$ & & & $\mathrm{~N}$ v/O vI & $-1.02 \pm 0.17$ \\
\hline & & C IV & $13.73 \pm 0.03 \pm 0.01$ & $52 \pm 5$ & $26 \pm 4$ & $13.80 \pm 0.04 \pm 0.01$ & $50 \pm 4$ & $27 \pm 3$ & C IV/O VI & $-0.05 \pm 0.09$ \\
\hline & & Si IV & $12.98 \pm 0.03 \pm 0.02$ & $53 \pm 6$ & $25 \pm 5$ & $12.88 \pm 0.07 \pm 0.02$ & & & Si IV/C IV & $-0.84 \pm 0.09$ \\
\hline & & $\begin{array}{l}\text { Fe III } \\
S_{\text {III }}\end{array}$ & $\begin{array}{c}13.56 \pm 0.09 \pm 0.10 \\
{\left[\mathrm{H}_{2}\right]}\end{array}$ & & & & & & $\mathrm{Fe}$ III/C IV & $-0.21 \pm 0.10$ \\
\hline \multirow[t]{4}{*}{ PKS0312-77 } & $-70: 40$ & O VI & [No flux] & & & [No flux] & & & & \\
\hline & & $\mathrm{N} \mathrm{v}$ & $<13.60$ & & & $<13.90$ & & & & \\
\hline & & $\mathrm{C}$ IV & $14.19 \pm 0.07 \pm 0.01$ & $-18 \pm 2$ & $57 \pm 5$ & $14.17 \pm 0.11 \pm 0.01$ & & & & \\
\hline & & $\begin{array}{l}\text { Si IV } \\
\text { Fe III } \\
\text { S III }\end{array}$ & $\begin{array}{c}13.49 \pm 0.06 \pm 0.02 \\
{[\text { Fe II }]} \\
{[\text { No flux }]}\end{array}$ & $-26 \pm 7$ & $57 \pm 13$ & $13.53 \pm 0.08 \pm 0.01$ & $-25 \pm 7$ & $57 \pm 10$ & $\mathrm{Si}$ IV/C IV & $-0.67 \pm 0.16$ \\
\hline \multirow[t]{5}{*}{ PKS0405-12 } & $-70: 55$ & O VI & $13.97 \pm 0.04 \pm 0.03$ & $1 \pm 4$ & $60 \pm 6$ & $14.01 \pm 0.06 \pm 0.05$ & $4 \pm 4$ & $56 \pm 6$ & & \\
\hline & & $\mathrm{N} \mathrm{v}$ & $<13.27$ & & & $<13.58$ & & & $\mathrm{~N}$ v/O vI & $<-0.72$ \\
\hline & & C IV & $14.09 \pm 0.03 \pm 0.01$ & $-16 \pm 1$ & $51 \pm 6$ & $14.05 \pm 0.05 \pm 0.01$ & $-6 \pm 7$ & $64 \pm 6$ & C IV/O VI & $0.08 \pm 0.09$ \\
\hline & & Si IV & $13.43 \pm 0.03 \pm 0.01$ & $-9 \pm 1$ & $53 \pm 5$ & $13.40 \pm 0.06 \pm 0.01$ & $0 \pm 4$ & $51 \pm 7$ & $\mathrm{Si}$ IV/C IV & $-0.66 \pm 0.09$ \\
\hline & & $\mathrm{Fe}$ III & $14.14 \pm 0.04 \pm 0.03$ & $-2 \pm 2$ & $55 \pm 5$ & & & & $\mathrm{Fe}$ III/C IV & $0.07 \pm 0.07$ \\
\hline
\end{tabular}


Table 2

(Continuted)

\begin{tabular}{|c|c|c|c|c|c|c|c|c|c|c|}
\hline \multirow[t]{2}{*}{ Object } & \multirow{2}{*}{$\begin{array}{l}\text { Vel. Range } \\
\left(\mathrm{km} \mathrm{s}^{-1}\right)\end{array}$} & \multirow[t]{2}{*}{ Ion } & \multicolumn{3}{|c|}{ Strong Line } & \multicolumn{3}{|c|}{ Weak Line } & \multicolumn{2}{|c|}{ Ratios } \\
\hline & & & $\begin{array}{c}\log N \\
\left(\mathrm{~cm}^{-2}\right) \\
(4)\end{array}$ & $\begin{array}{c}\langle v\rangle \\
\left(\mathrm{km} \mathrm{s}^{-1}\right) \\
(5)\end{array}$ & $\begin{array}{c}\text { FWHM } \\
\left(\mathrm{km} \mathrm{s}^{-1}\right) \\
(6)\end{array}$ & $\begin{array}{c}\log N \\
\left(\mathrm{~cm}^{-2}\right) \\
(7)\end{array}$ & $\begin{array}{c}\langle v\rangle \\
\left(\mathrm{km} \mathrm{s}^{-1}\right) \\
(8)\end{array}$ & $\begin{array}{c}\text { FWHM } \\
\left(\mathrm{km} \mathrm{s}^{-1}\right) \\
(9)\end{array}$ & $\begin{array}{l}\text { Ions } \\
\text { (10) }\end{array}$ & $\begin{array}{c}\log \text { Ratio } \\
\text { (11) }\end{array}$ \\
\hline (1) & & S III & $14.55 \pm 0.04 \pm 0.07$ & & & & & & $\mathrm{~S}$ III/C IV & $0.48 \pm 0.07$ \\
\hline \multirow[t]{14}{*}{ PKS2005-489 } & $-110: 110$ & $\mathrm{O}_{\mathrm{VI}}$ & $14.73 \pm 0.02 \pm 0.01$ & $26 \pm 3$ & $111 \pm 3$ & {$[\mathrm{CII} *-\mathrm{GC}]$} & & & & \\
\hline & & $\mathrm{N} \mathrm{v}$ & $13.97 \pm 0.02 \pm 0.01$ & $30 \pm 4$ & $118 \pm 3$ & $13.97 \pm 0.02 \pm 0.01$ & $33 \pm 4$ & $115 \pm 4$ & $\mathrm{~N}$ v/O vI & $-0.76 \pm 0.04$ \\
\hline & & S III & $14.97 \pm 0.03 \pm 0.04$ & & & & & & & \\
\hline & $-110:-50$ & O VI & $13.67 \pm 0.05 \pm 0.06$ & & & {$[\mathrm{CII} *-\mathrm{GC}]$} & & & & \\
\hline & & $\mathrm{N} \mathrm{v}$ & $12.90 \pm 0.03 \pm 0.05$ & & & $12.85 \pm 0.06 \pm 0.08$ & & & $\mathrm{~N}$ v/O vI & $-0.79 \pm 0.09$ \\
\hline & & $\begin{array}{l}\mathrm{Fe} \text { III } \\
\mathrm{S}_{\text {III }}\end{array}$ & $\begin{aligned} 13.97 & \pm 0.05 \pm 0.04 \\
& <13.80\end{aligned}$ & $-79 \pm 6$ & $33 \pm 3$ & & & & & \\
\hline & $-50: 50$ & O VI & $14.48 \pm 0.02 \pm 0.02$ & & & $14.51 \pm 0.03 \pm 0.02$ & & & & \\
\hline & & $\mathrm{N} \mathrm{v}$ & $13.66 \pm 0.02 \pm 0.02$ & & & $13.67 \pm 0.02 \pm 0.02$ & & & $\mathrm{~N}$ v/O vI & $-0.83 \pm 0.05$ \\
\hline & & Fe III & $14.26 \pm 0.04 \pm 0.03$ & & & & & & & \\
\hline & & S III & $14.67 \pm 0.03 \pm 0.04$ & & & & & & & \\
\hline & $50: 110$ & O VI & $14.30 \pm 0.03 \pm 0.03$ & & & $14.29 \pm 0.03 \pm 0.04$ & & & & \\
\hline & & $\mathrm{N} \mathrm{v}$ & $13.61 \pm 0.02 \pm 0.02$ & & & $13.62 \pm 0.02 \pm 0.03$ & & & $\mathrm{~N} v / \mathrm{O} v \mathrm{I}$ & $-0.67 \pm 0.05$ \\
\hline & & Fe III & $14.22 \pm 0.03 \pm 0.03$ & & & & & & & \\
\hline & & S III & $14.65 \pm 0.03 \pm 0.07$ & & & & & & & \\
\hline \multirow[t]{18}{*}{ PKS2155-304 } & $-55: 75$ & O VI & $14.25 \pm 0.02 \pm 0.02$ & $18 \pm 5$ & $80 \pm 2$ & $14.22 \pm 0.02 \pm 0.03$ & $16 \pm 6$ & $79 \pm 2$ & & \\
\hline & $-55: 120$ & $\mathrm{~N} \mathrm{v}$ & {$[\operatorname{Ly} \alpha]$} & & & $<13.40$ & & & & \\
\hline & $-55: 75$ & C IV & $14.04 \pm 0.02 \pm 0.01$ & $14 \pm 3$ & $77 \pm 3$ & $13.97 \pm 0.03 \pm 0.01$ & $17 \pm 3$ & $73 \pm 3$ & & \\
\hline & & Si IV & $13.33 \pm 0.02 \pm 0.01$ & $2 \pm 3$ & $76 \pm 3$ & $13.30 \pm 0.04 \pm 0.01$ & $0 \pm 3$ & $67 \pm 6$ & & \\
\hline & & $\mathrm{Fe}$ III & $13.96 \pm 0.03 \pm 0.03$ & $5 \pm 2$ & $57 \pm 6$ & & & & & \\
\hline & & S III & $14.13 \pm 0.04 \pm 0.06$ & $-6 \pm 2$ & $43 \pm 10$ & & & & & \\
\hline & $-55: 20$ & O VI & $13.95 \pm 0.02 \pm 0.04$ & & & $13.94 \pm 0.03 \pm 0.06$ & & & & \\
\hline & & $\mathrm{N} \mathrm{v}$ & {$[\operatorname{Ly} \alpha]$} & & & $<13.26$ & & & $\mathrm{~N}$ v/O vi & $<-0.69$ \\
\hline & & C IV & $13.77 \pm 0.03 \pm 0.01$ & $-12 \pm 7$ & $46 \pm 7$ & $13.66 \pm 0.05 \pm 0.02$ & $-10 \pm 9$ & $47 \pm 8$ & $\mathrm{C}$ IV/O VI & $-0.24 \pm 0.07$ \\
\hline & & Si IV & $13.17 \pm 0.02 \pm 0.01$ & $-16 \pm 5$ & $44 \pm 4$ & $13.17 \pm 0.04 \pm 0.01$ & $-14 \pm 4$ & $39 \pm 5$ & $\mathrm{Si} \mathrm{IV/C} \mathrm{IV}$ & $-0.54 \pm 0.07$ \\
\hline & & Fe III & $13.84 \pm 0.03 \pm 0.05$ & $-7 \pm 4$ & $29 \pm 6$ & & & & $\mathrm{Fe}$ III/C IV & $0.13 \pm 0.07$ \\
\hline & & S III & $14.12 \pm 0.03 \pm 0.06$ & $-10 \pm 3$ & $33 \pm 5$ & & & & $\mathrm{~S}$ III/C IV & $0.41 \pm 0.07$ \\
\hline & $20: 75$ & O VI & $13.96 \pm 0.02 \pm 0.04$ & & & $13.92 \pm 0.02 \pm 0.06$ & & & & \\
\hline & & $\mathrm{N} \mathrm{v}$ & $<12.92$ & & & $<13.19$ & & & $\mathrm{~N}$ v/O vi & $<-1.02$ \\
\hline & & C IV & $13.73 \pm 0.02 \pm 0.02$ & $43 \pm 5$ & $35 \pm 1$ & $13.71 \pm 0.04 \pm 0.02$ & $41 \pm 5$ & $34 \pm 2$ & $\mathrm{C}$ IV/O VI & $-0.22 \pm 0.05$ \\
\hline & & Si IV & $12.85 \pm 0.04 \pm 0.02$ & & & $12.71 \pm 0.11 \pm 0.03$ & & & Si IV/C IV & $-0.94 \pm 0.13$ \\
\hline & & $\mathrm{Fe}$ III & $13.35 \pm 0.08 \pm 0.14$ & & & & & & & \\
\hline & & S III & $<13.31$ & & & & & & $\mathrm{~S}$ III/C IV & $<-0.41$ \\
\hline \multirow[t]{4}{*}{ RX J0100.4-5113 } & $-50: 60$ & O VI & $14.18 \pm 0.06 \pm 0.03$ & $24 \pm 5$ & $42 \pm 12$ & $14.33 \pm 0.07 \pm 0.04$ & & & & \\
\hline & & $\mathrm{N} \mathrm{v}$ & $<13.13$ & & & $<13.55$ & & & $\mathrm{~N}$ v/O vI & $<-1.13$ \\
\hline & & $\mathrm{Fe}$ III & $14.17 \pm 0.08 \pm 0.03$ & $2 \pm 3$ & $58 \pm 4$ & & & & & \\
\hline & & S III & $14.38 \pm 0.12 \pm 0.04$ & $3 \pm 5$ & $60 \pm 7$ & & & & & \\
\hline \multirow[t]{3}{*}{ RX J1830.3+7312 } & $-70: 30$ & O VI & $14.33 \pm 0.09 \pm 0.02$ & $-28 \pm 5$ & $43 \pm 6$ & {$[14.38 \pm 0.10 \pm 0.04]$} & & & & \\
\hline & & $\mathrm{N} \mathrm{v}$ & {$[13.14 \pm 0.04 \pm 0.04]$} & & & {$[13.39 \pm 0.05 \pm 0.03]$} & & & & \\
\hline & & $\begin{array}{l}\mathrm{Fe} \text { III } \\
\mathrm{S}_{\text {III }}\end{array}$ & $\begin{array}{c}14.22 \pm 0.07 \pm 0.03 \\
{\left[\mathrm{H}_{2}\right]}\end{array}$ & $-34 \pm 5$ & $47 \pm 9$ & & & & & \\
\hline \multirow[t]{4}{*}{ Ton S180 } & $-80: 75$ & O VI & $14.33 \pm 0.03 \pm 0.01$ & $-10 \pm 3$ & $72 \pm 5$ & $14.31 \pm 0.06 \pm 0.03$ & $-11 \pm 4$ & $80 \pm 6$ & & \\
\hline & & $\mathrm{N} \mathrm{v}$ & $13.12 \pm 0.07 \pm 0.02$ & $-15 \pm 7$ & $82 \pm 15$ & $<13.24$ & & & $\mathrm{~N}$ v/O vI & $-1.20 \pm 0.10$ \\
\hline & & Fe III & $14.05 \pm 0.07 \pm 0.03$ & $-9 \pm 5$ & $77 \pm 9$ & & & & & \\
\hline & & S III & $14.18 \pm 0.12 \pm 0.06$ & $-14 \pm 8$ & $76 \pm 16$ & & & & & \\
\hline \multirow[t]{6}{*}{ Ton S210 } & $-75: 85$ & O VI & $14.49 \pm 0.02 \pm 0.01$ & & & $14.46 \pm 0.03 \pm 0.02$ & & & & \\
\hline & & $\mathrm{N} \mathrm{v}$ & $13.56 \pm 0.13 \pm 0.01$ & $-3 \pm 7$ & $86 \pm 16$ & $<13.91$ & & & $\mathrm{~N}$ v/O vI & $-0.91 \pm 0.14$ \\
\hline & & C IV & $14.38 \pm 0.04 \pm 0.01$ & $-8 \pm 4$ & $90 \pm 4$ & $14.31 \pm 0.06 \pm 0.01$ & $-15 \pm 6$ & $86 \pm 6$ & $\mathrm{C}$ IV/O VI & $-0.12 \pm 0.08$ \\
\hline & & Si IV & $13.61 \pm 0.04 \pm 0.01$ & $-13 \pm 2$ & $74 \pm 5$ & [Unid. Contam.] & & & $\mathrm{Si} \mathrm{IV/C} \mathrm{IV}$ & $-0.74 \pm 0.08$ \\
\hline & & Fe III & $14.01 \pm 0.06 \pm 0.03$ & $-6 \pm 3$ & $74 \pm 10$ & & & & $\mathrm{Fe}$ III/C IV & $-0.34 \pm 0.09$ \\
\hline & & S III & $14.36 \pm 0.07 \pm 0.14$ & & & & & & & \\
\hline UGC12163 & $-90: 70$ & O VI & $14.12 \pm 0.10 \pm 0.03$ & & & $14.29 \pm 0.12 \pm 0.04$ & & & & \\
\hline & & $\mathrm{N} \mathrm{v}$ & $<13.60$ & & & $<13.64$ & & & $\mathrm{~N}$ v/O vI & $<-0.61$ \\
\hline & & $\mathrm{C}$ IV & $14.34 \pm 0.06 \pm 0.01$ & & & $14.30 \pm 0.12 \pm 0.01$ & $-10 \pm 6$ & $72 \pm 13$ & C IV/O VI & $0.11 \pm 0.21$ \\
\hline & & Si IV & $13.82 \pm 0.06 \pm 0.01$ & $-8 \pm 1$ & $72 \pm 6$ & [Unid. Contam.] & & & Si IV/C IV & $-0.50 \pm 0.14$ \\
\hline & & Fe III & $14.44 \pm 0.07 \pm 0.02$ & $11 \pm 6$ & $89 \pm 7$ & & & & $\mathrm{Fe}$ III/C IV & $0.12 \pm 0.15$ \\
\hline & & S III & $14.85 \pm 0.09 \pm 0.13$ & & & & & & $\mathrm{~S}$ III/C IV & $0.53 \pm 0.16$ \\
\hline VIIZw118 & $-125: 60$ & O VI & $14.29 \pm 0.03 \pm 0.01$ & $-28 \pm 2$ & $95 \pm 3$ & {$\left[\mathrm{CII}^{*}\right]$} & & & & \\
\hline & & $\mathrm{N} \mathrm{v}$ & $13.19 \pm 0.07 \pm 0.02$ & & & $<13.35$ & & & $\mathrm{~N}$ v/O vI & $-1.10 \pm 0.08$ \\
\hline & & $\mathrm{Fe}$ III & $14.31 \pm 0.02 \pm 0.02$ & $-29 \pm 8$ & $83 \pm 15$ & & & & & \\
\hline
\end{tabular}


Table 2

(Continuted)

\begin{tabular}{|c|c|c|c|c|c|c|c|c|c|c|}
\hline \multirow[t]{2}{*}{ Object } & \multirow{2}{*}{$\begin{array}{l}\text { Vel. Range } \\
\left(\mathrm{km} \mathrm{s}^{-1}\right)\end{array}$} & \multirow[t]{2}{*}{ Ion } & \multicolumn{3}{|c|}{ Strong Line } & \multicolumn{3}{|c|}{ Weak Line } & \multicolumn{2}{|c|}{ Ratios } \\
\hline & & & $\begin{array}{c}\log N \\
\left(\mathrm{~cm}^{-2}\right) \\
(4)\end{array}$ & $\begin{array}{c}\langle v\rangle \\
\left(\mathrm{km} \mathrm{s}^{-1}\right) \\
(5)\end{array}$ & $\begin{array}{l}\text { FWHM } \\
\left(\mathrm{km} \mathrm{s}^{-1}\right) \\
(6)\end{array}$ & $\begin{array}{c}\log N \\
\left(\mathrm{~cm}^{-2}\right) \\
(7)\end{array}$ & $\begin{array}{c}\langle v\rangle \\
\left(\mathrm{km} \mathrm{s}^{-1}\right) \\
(8)\end{array}$ & $\begin{array}{c}\text { FWHM } \\
\left(\mathrm{km} \mathrm{s}^{-1}\right) \\
(9)\end{array}$ & $\begin{array}{l}\text { Ions } \\
\text { (10) }\end{array}$ & $\begin{array}{c}\log \text { Ratio } \\
\text { (11) }\end{array}$ \\
\hline & $-125:-40$ & $\begin{array}{l}\text { S III } \\
\text { O VI }\end{array}$ & $\begin{array}{c}{\left[\mathrm{H}_{2}\right]} \\
13.91 \pm 0.04 \pm 0.04\end{array}$ & & & {$\left[\mathrm{CII}^{*}\right]$} & & & & \\
\hline & & $\begin{array}{l}\mathrm{N} \text { v } \\
\text { Fe III } \\
\text { S III }\end{array}$ & $\begin{array}{c}13.11 \pm 0.06 \pm 0.02 \\
13.91 \pm 0.04 \pm 0.06 \\
{\left[\mathrm{H}_{2}\right]}\end{array}$ & $-77 \pm 4$ & $46 \pm 3$ & $<13.06$ & & & $\mathrm{~N}$ v/O vI & $-0.80 \pm 0.07$ \\
\hline & $-40: 60$ & $\mathrm{O}$ VI & $14.06 \pm 0.03 \pm 0.03$ & & & {$\left[\mathrm{H}_{2}\right]$} & & & & \\
\hline & & $\begin{array}{l}\mathrm{N} \text { V } \\
\mathrm{Fe} \text { III } \\
\mathrm{S} \text { III }\end{array}$ & $\begin{array}{c}<12.78 \\
14.10 \pm 0.03 \pm 0.03 \\
{\left[\mathrm{H}_{2}\right]}\end{array}$ & & & $<13.16$ & & & $\mathrm{~N}$ v/O vI & $<-1.28$ \\
\hline
\end{tabular}

Note.

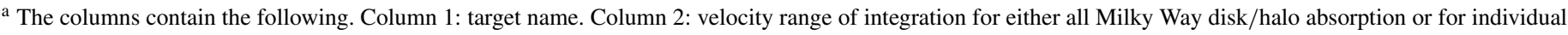

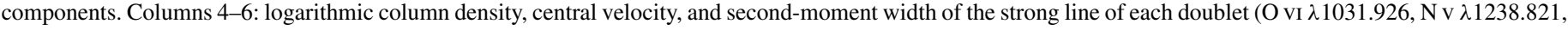

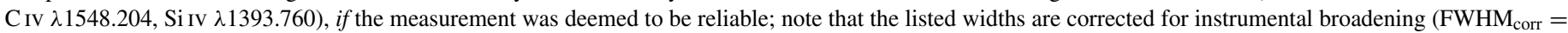

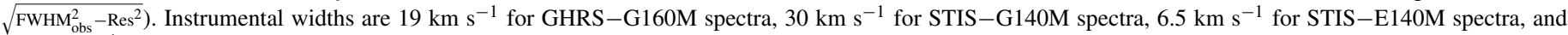

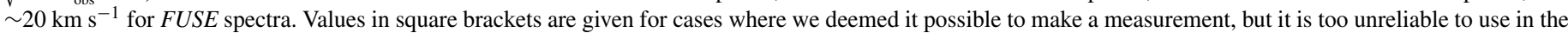

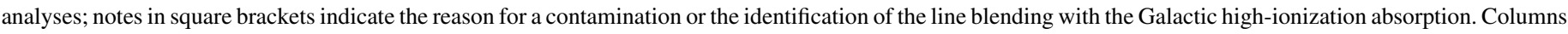

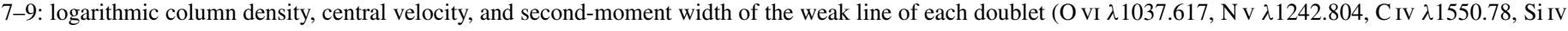

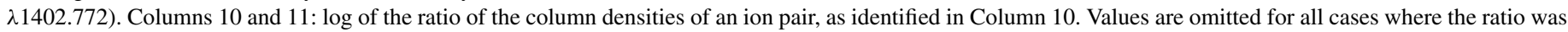
deemed untrustworthy.

(This table is available in its entirety in a machine-readable form in the online journal.)

We checked all doublet measurements for consistency. The two measurements for each half of each doublet were deemed to be compatible if the column density difference was less than the sum of the statistical and systematic errors for each individual component. For example, for the -110 to $+105 \mathrm{~km} \mathrm{~s}^{-1}$ integration toward 3C 273.0, the $\mathrm{Nv}$ lines give column densities of $13.94 \pm 0.02 \pm 0.01$ and $13.90 \pm 0.03 \pm 0.01$. As 13.94 and 13.90 are closer together than $0.02+0.01+0.03+0.01$ $=0.07 \mathrm{dex}$, these two values are deemed compatible. We justify adding the errors linearly instead of in quadrature by noting that just by chance the measurement for a particular line will be more than $1 \sigma$ above (or below) the real value in $16 \%$ of the cases. In $16 \%$ of those cases the measurement of the other line of the doublet will be more than $1 \sigma$ off in the other direction. Thus, with 258 cases where we measure both members of a doublet, we expect 6.6 cases where the column densities implied by the two member of the doublet differ by more than the sum of the errors. We find seven such cases; in four of these the excess in the difference is just $0.01 \mathrm{dex}$. In only one case do the two lines of the doublet suggest the presence of saturation. This is for the $\mathrm{C}$ IV lines in the direction toward ESO 141-G55, which are very deep, resulting in a factor-of-two difference in the column densities derived from the two members of the doublet. Since our check shows that in all but one case the absorption lines are unlikely to be saturated, we estimated final values for the ionic column densities by averaging the measurements toward the two lines of the doublet, if both measurements were deemed to be useful. There is one exception: for the saturated $\mathrm{C}$ IV line toward ESO 141-G55, we instead used the rule-of-thumb derived by Savage \& Sembach (1991) that $\log N($ ion $)=2 \log N$ (weak) $\log N$ (strong).

The resulting measurements are presented in Table 2. This table shows for each target, and for each component, the measured column densities in each high-ionization line, as well as the central velocities, widths, and ratios for those cases where the result was deemed to be reasonably reliable. Note that the widths that we list are corrected for instrumental broadening (see notes for details).

We compared our values to those listed by Savage et al. (1997a) in their earlier study of highly ionized atoms toward extragalactic targets and find that for the most part our measurements of $\mathrm{N} \mathrm{v}$ and $\mathrm{C}$ IV column densities are the same within the errors. There are two exceptions: (1) H 1821+643, for which Savage et al. (1997a) had not realized that there is strong contamination associated with the nearby planetary nebula K1-16 and (2) PKS 2155-304, for which we concluded that the $\mathrm{N} \mathrm{v}$ $\lambda 1238.821$ line is contaminated by an intergalactic Ly $\alpha$ line because the two lines of the doublet do not match, and the absorption that might be Galactic $\mathrm{Nv}$ extends to much more negative velocities than is the case for the other high-ionization lines.

We also checked our measurements against the numbers presented by Indebetouw \& Shull (2004b). To make their measurements, they integrated all of the high-ionization absorption, mixing together HVCs and Milky Way thick disk components. In a few cases they did not remove blending intergalactic absorption and they did not remove the absorption associated with the planetary nebula K1-16 near the H 1821+643 sight line. Furthermore, their velocity limits are often inconsistent with our judgments. Also, in many cases the column densities they give for the two lines in a $\mathrm{N} \mathrm{v}$ or $\mathrm{C}$ IV doublet are often rather different, sometimes by as much as 0.3 dex. In addition, the column density errors in their table are much larger than what we found (typically they are listed as $0.2-0.6 \mathrm{dex}$ ). Finally, in sight lines where there are no HVCs or other problems the column densities listed by Indebetouw \& Shull (2004b) still usually differ from our results. Due to all these issues, we did not make a detailed comparison of our results to the measurements given by Indebetouw \& Shull (2004b). 


\section{RESULTS}

In this section, we analyze several different aspects of the highly ionized gas, including the kinematic properties of the absorption lines (extent, component width, relation to Galactic rotation), the distribution of column densities, the distribution of velocities and column densities across the sky, and the distribution of column density ratios. These analyses show that the gas in which these ions occur participates in differential galactic rotation, but has a large velocity dispersion. There is no evidence for a gradient in the rotation velocity with height above the Galactic plane. The highly ionized ions are present across the sky, in similar amounts in every direction, but with a patchy distribution. There are some well-delineated regions where the average properties are noticeably different (factor-of-two average enhancement around the Galactic center and near the Galactic north pole, O vi showing a factor-oftwo deficit at intermediate southern latitude). The column densities and column density ratios in individual parcels of transition temperature gas have a narrow distribution, suggesting a uniformity of its properties.

\subsection{Velocity Differences between Ions}

We compared the centroids of the absorption profiles of the different ions (O vi, Nv, C IV, Si IV, Fe III) to each other and found that they correlate very well. In particular, for the nine components where both the $\mathrm{O}$ VI and $\mathrm{N} v$ absorption lines are well defined and simple, the centroids have an average offset of $0 \pm 8 \mathrm{~km} \mathrm{~s}^{-1}$, i.e., the rms difference is well within the resolution of the FUSE O vi spectra, as well as within the alignment errors associated with the wavelength calibration of the FUSE data. For the 24 components with good data for both C IV and Si IV, the difference in their centroids is $0 \pm 3 \mathrm{~km} \mathrm{~s}^{-1}$. The centroids of $\mathrm{OVI}$ components are about $4 \pm 8 \mathrm{~km} \mathrm{~s}^{-1}$ more negative than those of C IV, Si IV, and Fe III, which is not a significant difference. The $\mathrm{NV}$ and $\mathrm{C}$ IV lines appear to be well aligned (average difference $-2 \pm 4 \mathrm{~km} \mathrm{~s}^{-1}$ for seven components), and all of the cases where both of these lines are seen are derived from STIS-E140M spectra. Thus, the preponderance of the evidence suggests that there are no velocity offsets between the centroids of the absorption lines of the different highly ionized species.

\subsection{Velocity Extent of the Galactic Absorption}

In each direction, there is a range of velocities that is compatible with differential galactic rotation. Following Wakker (2004), we estimate this range using a model in which gas rotates at $220 \mathrm{~km} \mathrm{~s}^{-1}$ around the center of the Milky Way for galactocentric radii $0.5-26 \mathrm{kpc}$ in a layer with thickness of $3 \mathrm{kpc}$ inside the $8.0 \mathrm{kpc}$ solar radius, increasing to $6 \mathrm{kpc}$ at $26 \mathrm{kpc}$ from the Galactic center. At radii $<0.5 \mathrm{kpc}$ the velocity of rotation is assumed to decrease linearly to $0 \mathrm{~km} \mathrm{~s}^{-1}$ at $R=0$. Since most of our targets are at high galactic latitude, this usually produces a narrow range from 0 to about plus or minus $10 \mathrm{~km} \mathrm{~s}^{-1}$, except for directions below $|b|=30^{\circ}$, where a range of 0 to about plus or minus $40 \mathrm{~km} \mathrm{~s}^{-1}$ is found.

Figure 3 shows the observed extents of the absorption profiles graphically. The vertical lines give the range of velocities over which Galactic absorption is seen, while the short horizontal lines are drawn at the median difference of $\pm 60 \mathrm{~km} \mathrm{~s}^{-1}$ more positive or negative than those the simple model predicts. As this figure shows, the observed range of velocities over which absorption is seen is much larger than that predicted by differential galactic rotation. Absorption typically extends to velocities that are $45-100 \mathrm{~km} \mathrm{~s}^{-1}$ more negative than expected, with an extreme of $125 \mathrm{~km} \mathrm{~s}^{-1}$ toward PG $0953+414$. At high latitudes $\left(b>45^{\circ}\right)$, the typical profile also extends to velocities that are $30-80 \mathrm{~km} \mathrm{~s}^{-1}$ more positive. Looking separately at positive and negative velocities for four sky quadrants $(b=$ $-90^{\circ}:-45^{\circ}, b=-45^{\circ}: 0^{\circ}, b=0^{\circ}: 45^{\circ}$, and $b=45^{\circ}: 90^{\circ}(10,10$, 9 , and 16 sight lines, respectively)), the median and the average deviation from the maximum negative or positive velocity predicted by the simple model of rotation is about $60 \mathrm{~km} \mathrm{~s}^{-1}$, except for negative velocities near the north polar cap, where the median and average are $90 \mathrm{~km} \mathrm{~s}^{-1}$. That is, for sight lines near the north Galactic pole there appears to be an excess of absorption at velocities more negative than $-60 \mathrm{~km} \mathrm{~s}^{-1}$.

The velocity range over which the Milky Way transition temperature gas absorption extends is likely proportional to the outflow velocity of the hot gas. A value of about $60 \mathrm{~km} \mathrm{~s}^{-1}$ is in line with the predictions of Houck \& Bregman (1990) for the formation velocities of neutral (i.e., cooled) clouds in a low-temperature $\left(5 \times 10^{5} \mathrm{~K}\right)$ Galactic fountain, with a density of $10^{-3} \mathrm{~cm}^{-3}$ and a (two-sided) flow rate of $0.4 M_{\odot} \mathrm{yr}^{-1}$. Statistically speaking, however, the absorption profiles have a similar extent at positive and negative velocities, independent of Galactic longitude or latitude. Thus, on average, vertical motions are as large as motions parallel to the plane. This suggests that the material is highly turbulent. The only evidence for a deviation from this pattern is that there appears to be a slight preference for infall in the north Galactic cap region. This may just be a temporary local occurrence in the solar neighborhood, however.

\subsection{Line Width Distribution}

In Figure 4, we look at the distribution of line widths, rather than at the total extent of the absorption. These widths are found by calculating the second moment $(b)$ of the $N_{a}(v)$ distribution, converting it into the FWHM (FWHM $=1.665 b)$, and correcting for instrumental broadening. The figure has two panels for each ion. In the top panel, we use the full absorption profile, while in the bottom panel we use the FWHM for individual components (see Section 3). Note that in about half of the sight lines only a single component can be discerned. Only components where the measured width was deemed reliable are included. This means that in some sight lines the width of the full profile can be measured, but the widths of the two or three components that make up the profile are individually unreliable. Thus, the total number of measurements of the full line width is larger than the number of well-measured components.

For many sight lines it is possible to separately calculate the widths of both members of the O VI, N v, C IV, and Si IV doublets. In every case the two widths are identical to within the errors. Therefore, we use only the width of the strong line for the histograms in Figure 4 (except when only the weak line was measurable). Widths are not calculated if the Galactic profile blends with high-velocity absorption and it is not clear where to place the boundary (for Mrk 205, Mrk 279, Mrk 817, Mrk 876, and NGC 3783). All cases where we deemed it possible to estimate a width are listed in Table 2 and used in Figure 4.

We do not explicitly show a figure that directly compares the widths of the ions in individual absorption components, but these correlate very well. That is, if $\mathrm{FWHM}(\mathrm{O} \mathrm{VI})$ is large, then FWHM $(\mathrm{N} v)$ is large. The correlation coefficient between the two widths is 0.97. FWHM(C IV) and FWHM(Si IV) also correlate closely (correlation coefficient 0.94 ). The correlation between $\mathrm{FWHM}(\mathrm{O}$ vI) or $\mathrm{FWHM}(\mathrm{Nv})$ and $\mathrm{FWHM}(\mathrm{CIV})$ or 


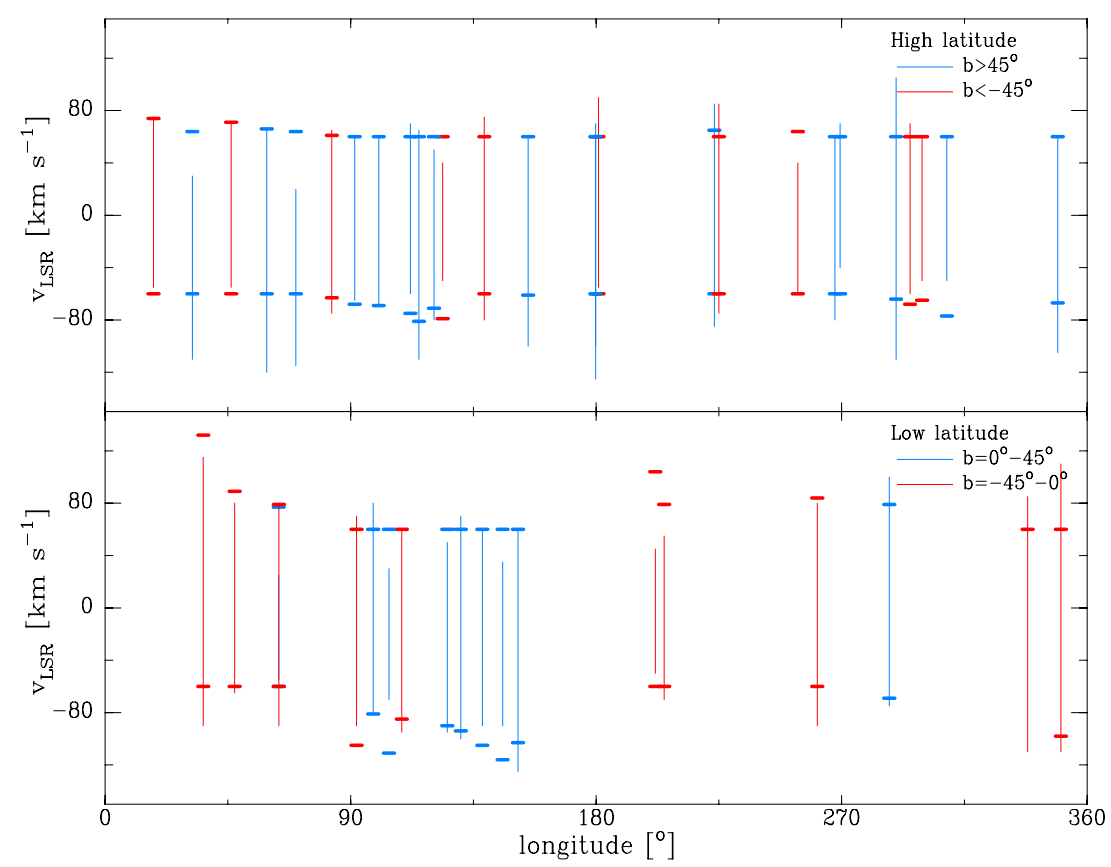

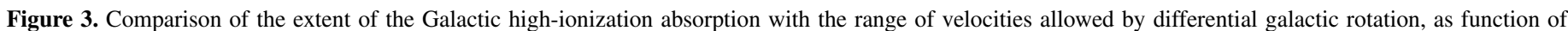

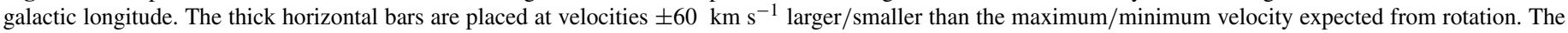

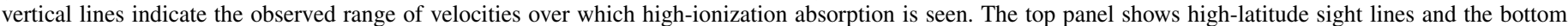
panel shows low-latitude sight lines, with northern sight lines in blue and southern sight lines in red.

$\mathrm{FWHM}(\mathrm{Si}$ IV) is not quite as good, but still strong $(\sim 0.92)$. Thus, $\mathrm{O}$ vi goes closely together with $\mathrm{N} v$, but somewhat less strongly with Civ and Si Iv, even though N v, Civ, and Si IV are determined from STIS-E140M spectra (with $6.5 \mathrm{~km} \mathrm{~s}^{-1}$ resolution), while O VI is seen in FUSE spectra $\left(20 \mathrm{~km} \mathrm{~s}^{-1}\right.$ resolution). Thus, the differences in line widths are not likely to be an artifact of the higher resolution of the STIS-E140M spectra.

As Figure 4 shows, the FWHM of the full Galactic absorption ranges from about $50 \mathrm{~km} \mathrm{~s}^{-1}$ to about $120 \mathrm{~km} \mathrm{~s}^{-1}$, with an average value in the range $60-80 \mathrm{~km} \mathrm{~s}^{-1}$, dependent on the ion. The widths of the $\mathrm{OVI}$ and $\mathrm{Nv}$ absorption lines appear similar, while those of C IV, Si IV, and Fe III appear to be slightly narrower. We applied a $t$-test to see whether these differences are statistically significant. This test is most useful when applied to the distributions of the widths of the individual components. Then the difference in the average line width of $\mathrm{O} v \mathrm{~V}$ and $\mathrm{Nv}$ $\left(59 \pm 18 \mathrm{~km} \mathrm{~s}^{-1}\right.$ and $62 \pm 20 \mathrm{~km} \mathrm{~s}^{-1}$ ) is not significant (the probability that they do not differ is $55 \%$ ). This is also the case for the difference between the average widths of $\mathrm{C}$ IV and $\mathrm{Si}$ IV $\left(42 \pm 11 \mathrm{~km} \mathrm{~s}^{-1}\right.$ and $43 \pm 13 \mathrm{~km} \mathrm{~s}^{-1}$, the probability that they do not differ is $80 \%$ ). However, the difference between each pair of ions is significant, with probabilities that the widths do not differ of $<0.5 \%$ when comparing O VI or $\mathrm{NV}$ to $\mathrm{C}$ IV or Si IV. The average width of the Fe III line $\left(50 \pm 19 \mathrm{~km} \mathrm{~s}^{-1}\right)$ is marginally larger than that of CIV (probability it does not differ 5\%), and insignificantly smaller than that of O VI (the probability it does not differ is $2 \%$ ). Thus, we cannot really tell whether Fe III belongs in the $\mathrm{C}$ IV $/ \mathrm{Si} \mathrm{IV} \mathrm{group} \mathrm{or} \mathrm{in} \mathrm{the} \mathrm{O}$ VI/ $\mathrm{N} \mathrm{v}$ group. Because of its lower ionization potential, the former is more likely, however. For S III the number of points is too small to say anything useful.

Thus, the evidence points to a situation in which the $\mathrm{O} v \mathrm{I}$ and $\mathrm{Nv}$ lines have similar widths, as do the CIV and Si IV lines, with the latter two ions having narrower lines on average. The general correlation of the line widths and the alignment in velocity between all ions (see Section 4.1) shows that the different ions must originate in the same general structures, but also that they cannot be completely cospatial. Furthermore, since even at a temperature of $10^{5} \mathrm{~K}$ the thermal width of the lightest of our elements (carbon) is only $20 \mathrm{~km} \mathrm{~s}^{-1}$, most of the observed line width cannot be due to thermal broadening. This implies that there is either a high level of turbulence or there are strong velocity gradients in the gas.

A difference in line widths between $\mathrm{OvI} / \mathrm{Nv}$ versus $\mathrm{CIV} /$ Si IV was previously found by Fox et al. (2003), who analyzed high-resolution $\left(3.5 \mathrm{~km} \mathrm{~s}^{-1}\right)$ STIS-E140H data of the halo star HD 116852. They saw both a narrow and a broad CIV and $\mathrm{Si}$ IV line, but $\mathrm{O}$ VI and $\mathrm{N} v$ did not show the narrow absorption. They interpreted this as indicating that there are regions in the thick disk that are sufficiently warm to contain C IV, but not warm enough to have $\mathrm{O}$ VI. In an analysis of 38 high-resolution STIS-E140H spectra of stars, Lehner et al. (2011) find the same effect in several other sight lines, with the average FWHM of the narrow C IV and Si IV components about $\sim 20 \mathrm{~km} \mathrm{~s}^{-1}$. They further find only broad absorption components for $\mathrm{O}$ VI and $\mathrm{N} \mathrm{V}$ (average FWHM $65 \pm 22$ and $50 \pm 25 \mathrm{~km} \mathrm{~s}^{-1}$, respectively). The $\mathrm{S} / \mathrm{N}$ of our data is usually not nearly as high as it is for these stellar spectra, making it more difficult to separate narrow and broad C IV/Si IV components. Furthermore, all of our sight lines pass through several kiloparsecs of gas, whereas the sight lines to the stars typically are only $1-3 \mathrm{kpc}$ long. This will produce more blending between structures at different distances/velocities for the AGN sight lines. Nevertheless, we find that the distributions of the widths of the $\mathrm{O} v \mathrm{VI}$ and $\mathrm{Nv}$ lines in sight lines through the thick disk are very similar to those in sight lines through the disk. We also find that the C IV/Si Iv lines are narrower, but we do not find the very narrow components seen in the disk. This is not necessarily a result of the poorer resolution of our spectra $\left(6.5 \mathrm{~km} \mathrm{~s}^{-1}\right.$ versus $\left.3.5 \mathrm{~km} \mathrm{~s}^{-1}\right)$, since lines that are $20 \mathrm{~km} \mathrm{~s}^{-1}$ wide should still have been resolved. Thus, the presence of narrow C IV/Si IV absorption components may indicate a real 

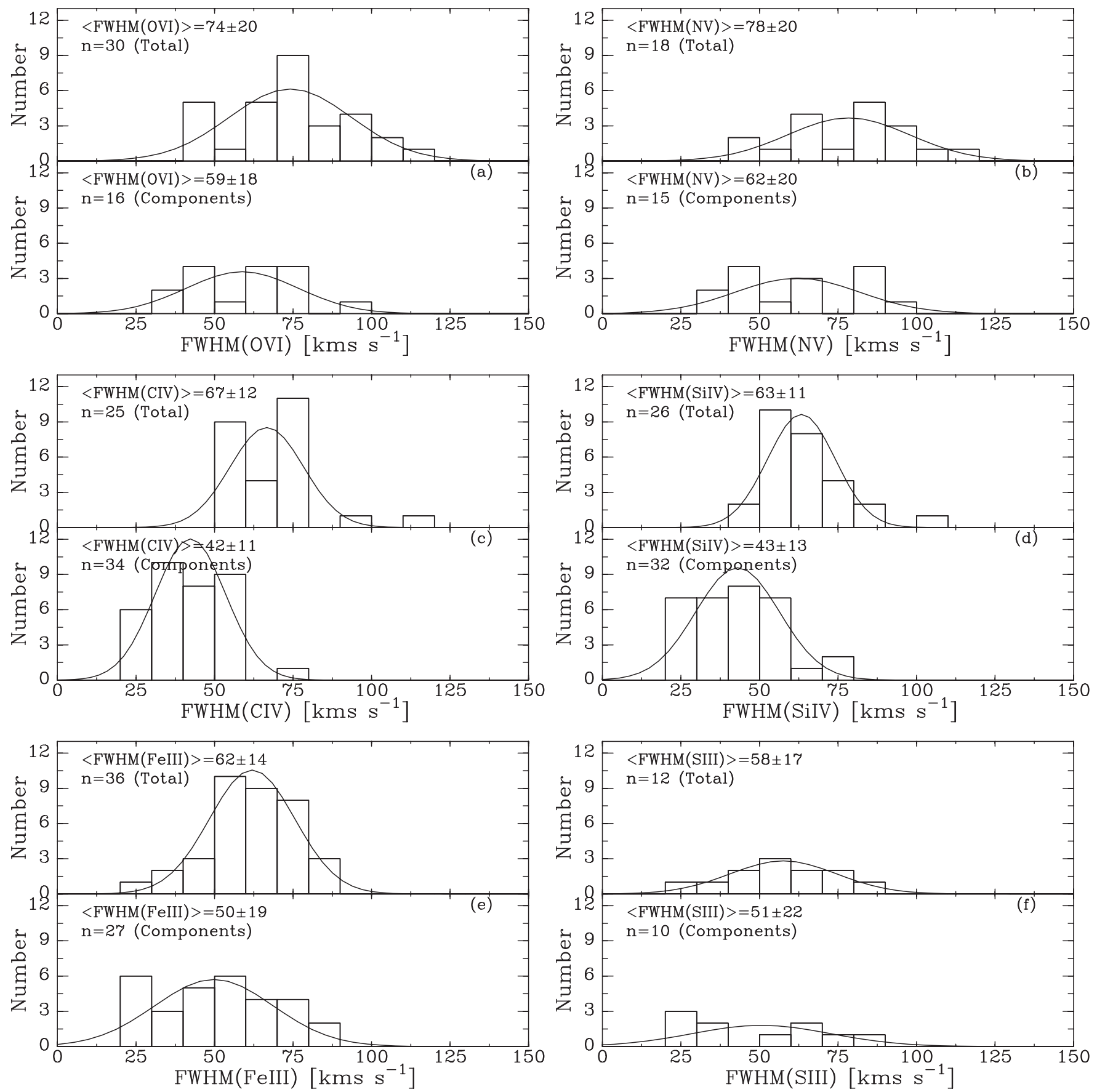

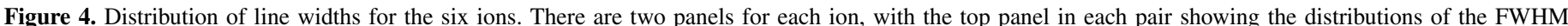

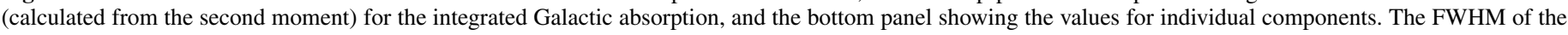

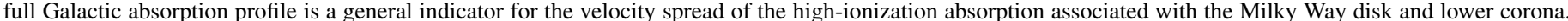

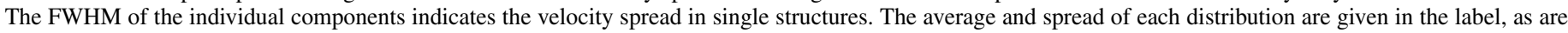
the number of sight lines/components that are used.

difference between low- and high-latitude sight lines. It may also be due to the longer path length through the disk for the AGN sight lines. Or it may be caused by the limitations of the lower $\mathrm{S} / \mathrm{N}$ in the extragalactic sight lines.

\subsection{Signature of Galactic Rotation}

In order to determine whether the velocity of the absorbing gas reflects Galactic rotation, we use the kinematical model described in Section 4.2, but add an exponential density distribution having scale height $2.6 \mathrm{kpc}$. We then find the velocity and density along the sight line in steps of $0.2 \mathrm{kpc}$, convert this density to an optical depth, and add a Gaussian with FWHM $60 \mathrm{~km} \mathrm{~s}^{-1}$. Summing over the path produces a predicted apparent column density profile, from which a centroid can be calculated. We look at two versions of this model, one in which the coronal gas corotates with the disk at all heights above the plane and one in which there is a decrease in the rotation velocity of $10 \mathrm{~km} \mathrm{~s}^{-1} \mathrm{kpc}^{-1}$. The latter is inspired by the results of, e.g., Fraternali et al. (2004), Heald et al. (2006, 2007), and Oosterloo et al. (2007), who find that in several nearby spiral galaxies $\mathrm{H} \alpha$ and $\mathrm{H}$ I emission show gradients of $\sim 15-25 \mathrm{~km} \mathrm{~s}^{-1} \mathrm{kpc}^{-1}$ at 


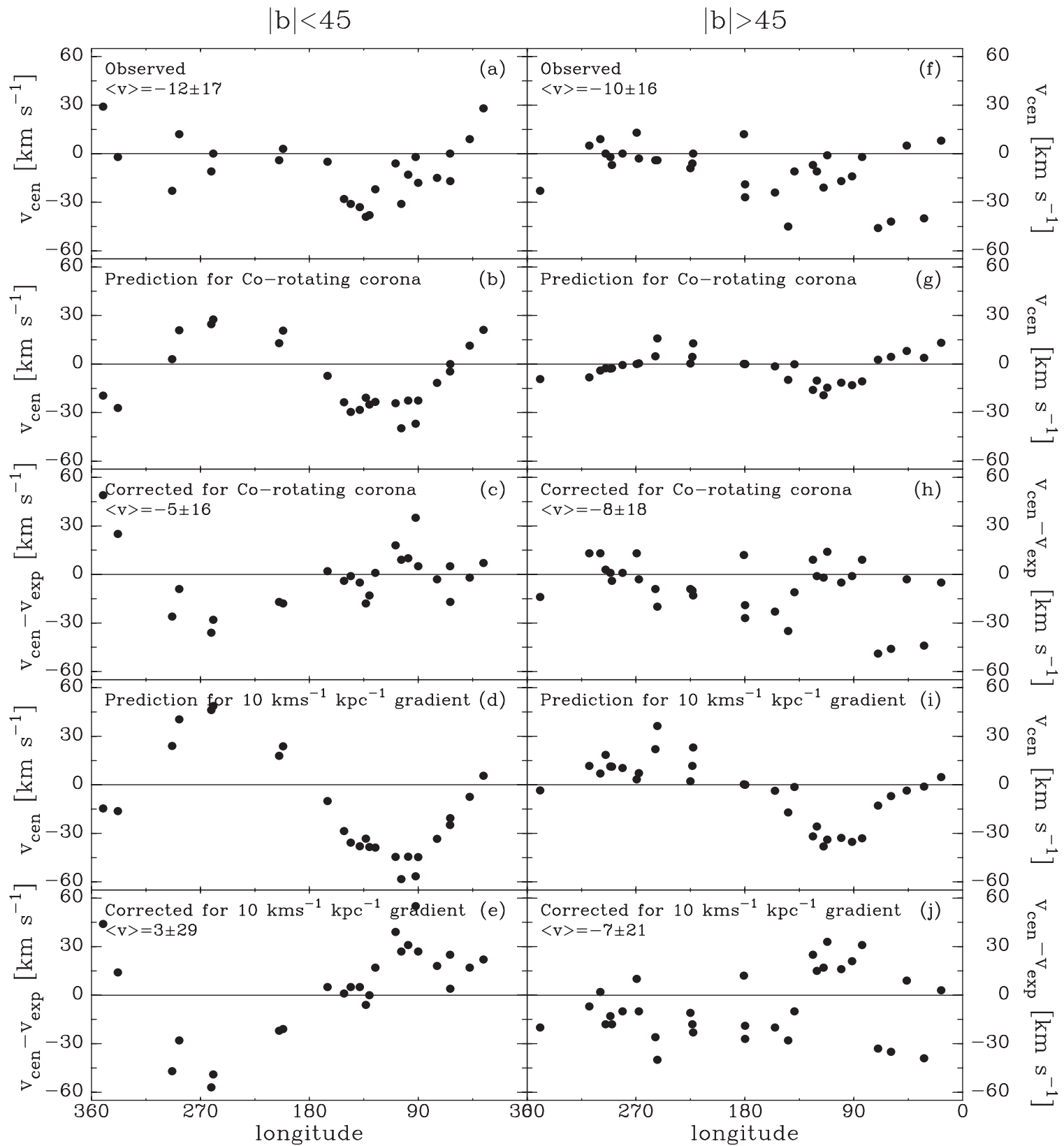

Figure 5. Comparison of observed and expected centroid velocities of the profiles of absorption by highly ionized atoms, as a function of longitude. Panels on the left are for sight lines at low latitudes $\left(|b|<45^{\circ}\right)$, panels on the right are for high latitudes. The top panels give the observations for $\mathrm{O}$ vi. The second and third panels give the predictions and difference between observed and predicted values for a corotating corona, while the bottom two panels give this for a corona having a $10 \mathrm{~km} \mathrm{~s}^{-1}$ decrease in velocity per kiloparsec away from the Galactic midplane.

heights more than $1 \mathrm{kpc}$ above the plane. Since in reality the distribution of the transition temperature gas is highly irregular, the only justified use of this model is to give an expectation value for the centroid of the absorption profile, but it cannot be used to model the individual absorption profiles.

Using our model, we find that Galactic rotation does have a subtle, but measurable effect on the centroids of the profiles. This can be seen in Figure 5, which shows the observations and the predictions for a corotating corona as well as those for a corona with a vertical velocity gradient. The differences between the observations and these two models are also shown. The panels on the left give this for low-latitude sight lines, while those on the right are valid for sight lines at high latitudes. For the high-latitude sight lines we expect to see little to no systematic variation with longitude in the centroid velocity, which is indeed observed to be the case. At low latitudes, the corotating corona predicts centroids to be about $-40 \mathrm{~km} \mathrm{~s}^{-1}$ near $l=90^{\circ}$, and about $+30 \mathrm{~km} \mathrm{~s}^{-1}$ near $l=270^{\circ}$, while the model with a $10 \mathrm{~km} \mathrm{~s}^{-1} \mathrm{kpc}^{-1}$ vertical velocity gradient predicts values of about -60 and $+60 \mathrm{~km} \mathrm{~s}^{-1}$, respectively. Increasing the gradient to $20 \mathrm{~km} \mathrm{~s}^{-1} \mathrm{kpc}^{-1}$ increased this range to about $\pm 70 \mathrm{~km} \mathrm{~s}^{-1}$. The observations appear more in line with the corotating corona version of the model. Excluding the two outliers at $l>300^{\circ}$ (ESO 141-G55 and PKS 2005-489), the average difference between predicted and observed centroids is $5 \mathrm{~km} \mathrm{~s}^{-1}$, with an rms spread of $16 \mathrm{~km} \mathrm{~s}^{-1}$ (see panel (c)). From Figure 5, we conclude that Galactic rotation does leave an imprint on the velocities of the highly ionized atoms. However, the effect is subtle, leading to centroids differing from $0 \mathrm{~km} \mathrm{~s}^{-1}$ by no more than $40 \mathrm{~km} \mathrm{~s}^{-1}$. 

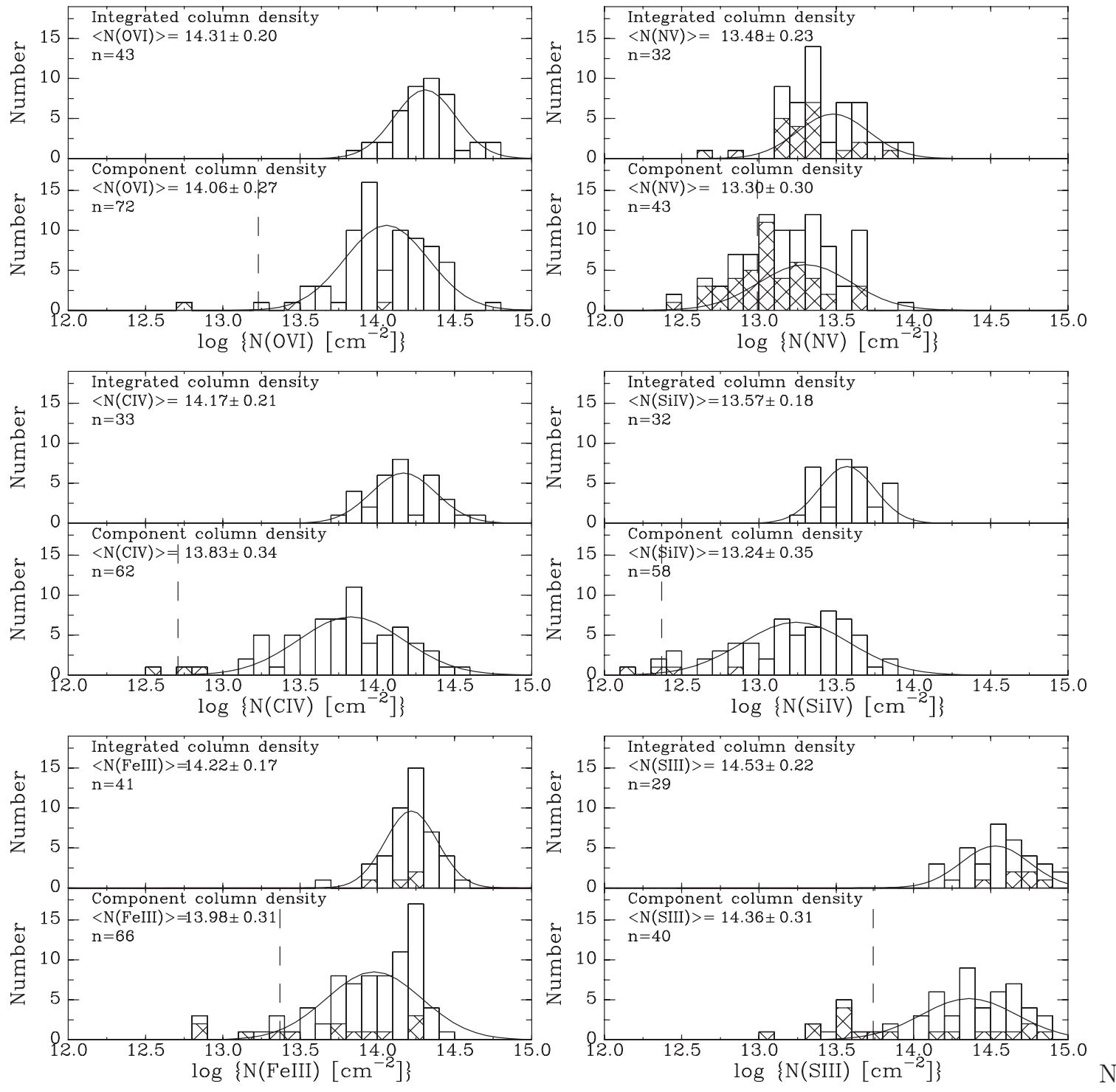

Figure 6. Distributions of column densities for the four high- and two intermediate-ionization species. The top panel of each pair is for the integrated column densities, the bottom panel for the individual components. The hatched parts of the histograms correspond to upper limits. The dotted vertical lines in the bottom panels show the detection limit for a $20 \mathrm{~m} \AA$ line with FWHM $40 \mathrm{~km} \mathrm{~s}^{-1}$, while the curves are Gaussians using the parameters listed in the label (i.e., number, average, and dispersion). Limits were excluded in these calculations (see the text for a discussion of why this is justified in the case of $\mathrm{N} \mathrm{v}$ ).

The assumed vertical distribution of the average density of the transition temperature has a large influence on the resulting predictions for the centroid velocities. If we were to assume that the density of the transition temperature gas is constant between 0 and several $(\sim 5) \mathrm{kpc}$ above the Galactic plane, we would predict very little difference between a corotating corona and one with a vertical gradient, but the centroids would have values up to $\pm 50 \mathrm{~km} \mathrm{~s}^{-1}$ (i.e., much larger than what is observed). If we instead assume a scale height of $0.5 \mathrm{kpc}$, the predicted centroids would range between $\pm 10 \mathrm{~km} \mathrm{~s}^{-1}$ for a corotating corona and between $\pm 30 \mathrm{~km} \mathrm{~s}^{-1}$ for one having a $10 \mathrm{~km} \mathrm{~s}^{-1} \mathrm{kpc}^{-1}$ vertical gradient. The predictions for the latter case are about as good as the ones for our standard model.

Thus, it is possible to exclude a model in which the transition temperature extends to very large heights above the plane. However, it is not really possible to differentiate between a model with a corotating corona and a scale height of $2.6 \mathrm{kpc}$ and a corona with a vertical velocity gradient and a scale height of $0.5 \mathrm{kpc}$. On the other hand, using the fact that the directly derived scale height is about $2.6 \mathrm{kpc}$, we can conclude that the transition temperature gas appears to be corotating up to heights of a few kiloparsecs.

\subsection{Column Density Distribution}

Figure 6 presents the distributions of the column densities for the different ions. For each ion we show the total Galactic column density in the top panel and the column densities of the individual components in the bottom panel. Upper limits are indicated by the hatched areas. In the bottom panel of each pair, the dotted vertical line shows the column density detection limit corresponding to a line with $20 \mathrm{~m} \AA$ equivalent width and FWHM $40 \mathrm{~km} \mathrm{~s}^{-1}$. Such an equivalent width is the detection limit when integrating over a $75 \mathrm{~km} \mathrm{~s}^{-1}$ window in data with an $\mathrm{S} / \mathrm{N}$ of about 20. These limits are 13.2 dex for O vI, 13.0 dex for $\mathrm{Nv}, 12.7$ dex for Civ, 12.4 dex for Si Iv, 13.4 dex for Fe III, and 13.7 dex for $\mathrm{S}$ III. Clearly, except for $\mathrm{N} v$, the typical detection limit is $0.6-1.1$ dex below the average component column density, making these lines always easily detectable. For $\mathrm{Nv}$ the detection limit is usually only about 0.3 dex below 
the typical value, which is mostly caused by the fact that the interstellar abundance of nitrogen is about 0.8 dex less than that of oxygen and carbon. Therefore, although $\mathrm{N} \mathrm{v}$ is detected in 43 Galactic components, for 46 components only an upper limit is possible. In contrast, an upper limit is found for O VI, C IV, and Si IV in just 3, 3, and 4 components, respectively.

Savage et al. (2003) derived an average Galactic O vi column density of $14.32 \pm 0.19$, which is basically the same as the value of $14.31 \pm 0.20$ found here. They also looked at the average value of $\log N(\mathrm{O} \mathrm{VI}) \sin |b|$, which is $14.21 \pm 0.23$, or about 0.11 dex lower. We do not show the distributions of $\log N\left(\mathrm{O}_{\mathrm{VI}}\right) \sin |b|$ separately, as they are very similar to the ones shown in Figure 6, but shifted to column densities that are $\sim 0.15$ dex lower. The spread in the column densities of highly ionized atoms is such that for $95 \%$ of the sight lines the integrated column density is within a factor $\sim 2.5$ from the average, i.e., not only is the transition temperature gas widespread, on average it is fairly evenly distributed.

The histograms in Figure 6 show that along paths through the Galactic thick disk, O VI, C IV, and Si IV are always found. Only $\mathrm{Nv}$ is sometimes missing, which is most likely due to the fact that the line is weaker, which in turn is due to the fact that the cosmic abundance of nitrogen is lower than that of oxygen and carbon. To calculate the average and dispersion of the distribution of $N(\mathrm{Nv})$, we only use the detections, and exclude the upper limits. We justify this on the basis of the fact that generally the ratio $\mathrm{N} v / \mathrm{O}$ i has a narrow range (see Section 4.8 below) and in sight lines where $\mathrm{N} \mathrm{v}$ is not detected the implied limit to the $\mathrm{Nv} / \mathrm{O}$ vi ratio is within the range of detected values or above it. That is, given $N(\mathrm{O} v \mathrm{VI})$ and $\mathrm{N} \mathrm{v} / \mathrm{O} \mathrm{VI}$, the non-detection of $\mathrm{N} \mathrm{v}$ is generally not significant. There are only 5 (out of 40) components for which the measured upper limit on the ratio $\mathrm{N} \mathrm{v/O} \mathrm{VI} \mathrm{is} \mathrm{slightly} \mathrm{more} \mathrm{than} 2 \sigma$ below the average ratio, i.e., based on $N(\mathrm{O} v \mathrm{~V})$, a detection of $\mathrm{N} \mathrm{v}$ would have been expected.

\subsection{Sky Distribution}

Savage et al. (2003) discovered that the O vi column density is systematically enhanced in the northern sky relative to the southern sky, but they did not see a systematic dependence of $N(\mathrm{O} \mathrm{VI})$ on longitude. To be precise, they found an average for $\log N(\mathrm{OVI}) \sin |b|$ of 14.26 for $b>0^{\circ}$ and 14.09 for $b<0^{\circ}$. We can now refine this conclusion.

In Figures 7 and 8, we show the logarithmic column densities of the six ions as a function of Galactic longitude. In Figure 8, we further include curves showing the expected change of column density with latitude for a plane-parallel layer (i.e., $\left.\log N\left(90^{\circ}\right) \sin |b|\right)$.

When looking at Figures 7 and 8 (at first ignoring the colors), it appears that sight lines with high column density occur relatively more often at longitudes within $30^{\circ}$ of $l=0^{\circ}$, with about a factor-of-two difference between directions near $l=$ $0^{\circ}$ versus directions near $l=180^{\circ}$. This effect is not seen in the column densities of Fe III or S III. Furthermore, sight lines at $b>45^{\circ}$ tend to lie above the blue $\log N\left(90^{\circ}\right) \sin |b|$ line. When exploring this further, we found that column densities are generally enhanced near the Galactic center and near the Galactic north pole. Furthermore, O vi is deficient in parts of the southern sky. These regions are delineated in Figure 9. We note that there are about 100 additional sight lines toward which $N(\mathrm{O}$ vI $)$ can be measured. These sight lines include the ones that were in the original FUSE O vi survey (Wakker et al. 2003) as well as sight lines observed between 2003 and 2007. We have not yet measured each of these with the same care as the sight lines in this paper, but a preliminary assessment shows that for these additional sight lines O VI column densities are also enhanced in the region of excess and depressed in the region of deficit defined by the sight lines measured in this paper. In Figures 7 and 8, we show the points in the region with enhanced column densities in red. It is clear from these figures that the excess column density seen in $\mathrm{O}$ VI in the region around the Galactic center is also seen in the other ions. Comparing the average $\log N \sin |b|$ for the region with an excess to those in the "normal" sky gives differences in the logarithm of the polar column densities of $0.20,0.36,0.31,0.26,0.21$, and 0.29 dex for O vi, N v, C IV, Si IV, Fe III, and S III, respectively. That column densities are enhanced over the Galactic center suggests the presence of enhanced activity. It is more difficult to explain the 0.17 dex deficit seen in $N(\mathrm{O} \mathrm{VI})$ in the southern sky, especially since it is not reflected in any of the other ions.

The total hydrogen column corresponding to each ion can be estimated from $\log <N \sin |b|>-\log A f$, with $\log <N \sin |b|>$ being the logarithm of the average polar column density (see Figure 8 for the values), $A$ the element abundance, and $f$ being the ionization fraction. The maximum ionization fraction of the ions is on the order of $20 \%$ at temperatures near $10^{5} \mathrm{~K}$ (see, e.g., Gnat \& Sternberg 2007). We take $f=10 \%$ and use solar abundances of $\log A=-3.31$ for oxygen, -3.57 for carbon, -4.17 for nitrogen, and -4.49 for silicon (Asplund et al. 2009). Then, the average polar column densities (logarithmic values 14.17, 13.22, and 13.94 for $\mathrm{O}$ VI, $\mathrm{N} \mathrm{V}$, and C IV, respectively) imply $\log N(\mathrm{H}$,eqv $) \sim 18.4$ for the transition temperature gas. This number can be compared to the total neutral hydrogen column density $\left(\log N\left(\mathrm{H}_{\mathrm{I}}\right) \sim 20.28\right)$ and total ionized hydrogen column density $\left(\log N\left(\mathrm{H}^{+}\right) \sim 20.0\right)$ (Savage \& Wakker 2009).

We note that Savage et al. (2003) compared the distribution of $\mathrm{O}$ VI column densities to that of the radio-continuum loops and X-ray emission. They found no obvious correlation. We rechecked these comparisons and we still see no clear relation between $N(\mathrm{O} \mathrm{vI})$ and other known structures. We also compared the pattern of the column densities and velocities to the distribution on the sky of the high- and intermediatevelocity H I clouds (see Wakker 2004 for sky maps of these objects). In the north, a large part of the sky at $l=100^{\circ}$ to $l-240^{\circ}, b>30^{\circ}$ is covered by negative-velocity gas $(v \sim-40$ to $-90 \mathrm{~km} \mathrm{~s}^{-1}$ ), in three structures called the "IntermediateVelocity Arch," the "Intermediate-Velocity Spur," and the "LowLatitude Intermediate-Velocity Arch" (as named by Kuntz \& Danly 1996). Although the O VI absorption in this part of the sky tends to have negative centroids, $\mathrm{O}$ VI absorption with negative centroids occurs both on and away from these H I structures, and there is no clear correlation. Furthermore, the region of sky where the excess high-ion column density is seen covers an area both overlapping with and away from the intermediate-velocity gas. However, some connection between the two phenomena would be logical, since the intermediate-velocity clouds (IVCs) are about $1 \mathrm{kpc}$ above the Galactic plane (Wakker 2001), which is similar to the scale height of the transition temperature gas. The connection clearly does not manifest itself as a simple correlation between the column densities of the neutral and transition temperature gas, but must be more complex. Understanding the connection will require many more sight lines toward which the highly ionized species are detected, and/or maps of the emission from these ions.

We further looked at the difference between absorption at negative velocities and absorption at positive velocities, 

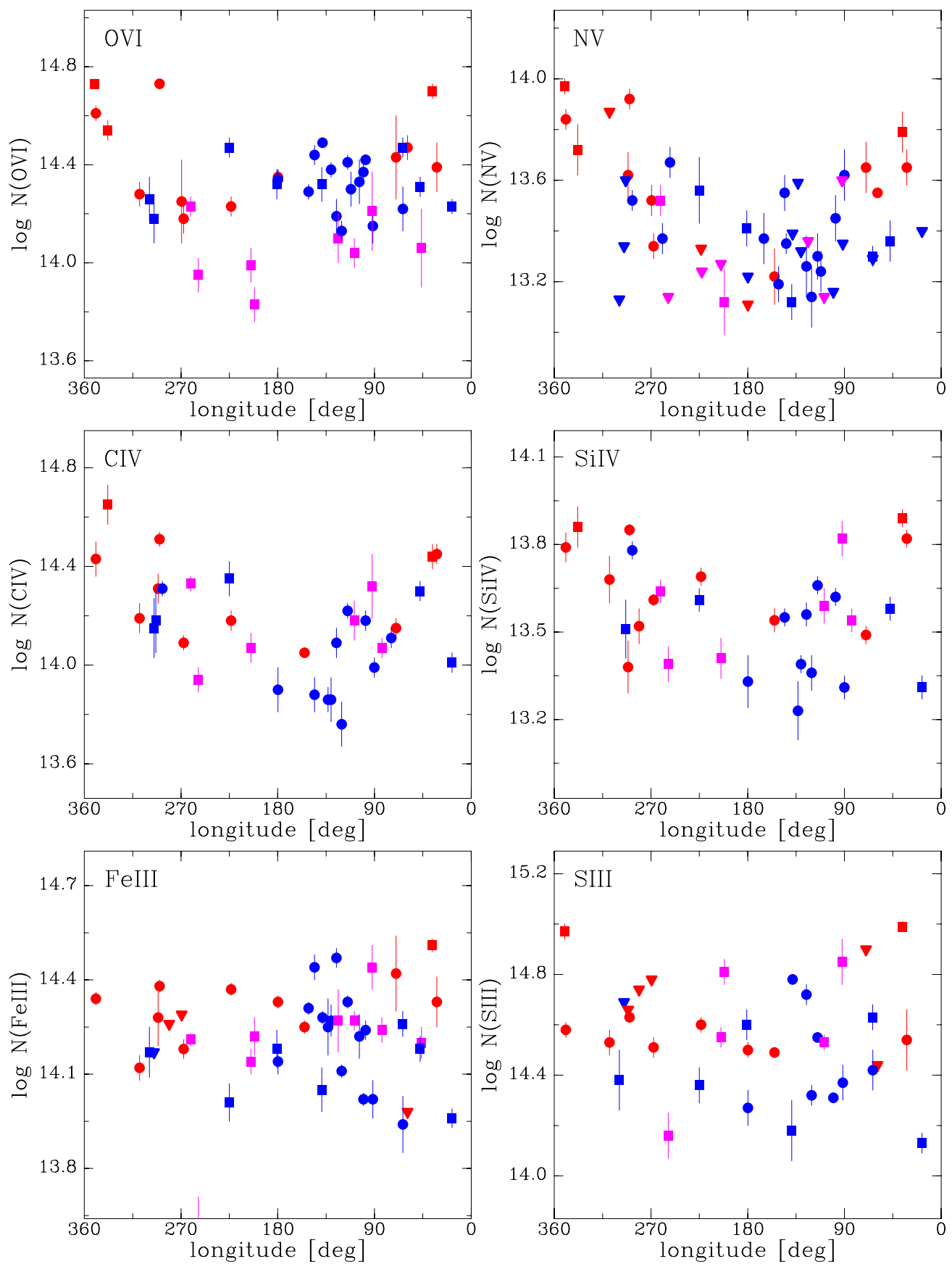

Figure 7. Scatter plot of ionic column densities against Galactic longitude. Circles are for sight lines with $b>0^{\circ}$; squares are for sight lines with $b<0^{\circ}$. Triangles correspond to upper limits. Red points are for sight lines where $N(\mathrm{O} v \mathrm{vI})$ is systematically enhanced (an area of sky delineated as $|l|<40^{\circ},|b|<50^{\circ}$ combined with $\left.|l|<80^{\circ}, b>40^{\circ}\right)$. Purple points are for sight lines showing a systematic deficit in $N\left(\mathrm{O} \mathrm{VI}\right.$ ) (approximately $l=70^{\circ}-280^{\circ}, b=-60^{\circ}$ to $-10^{\circ}$ ) and blue points cover the rest of the sky.

although the comparison suffers from having just 30-40 sight lines for which this can be done. We made this comparison in many ways, looking at northern versus southern sight lines, sight lines inside and outside the region of the O vi excess, etc., but we found only one (small) possible systematic difference. This is that the column densities at negative velocities appear to be marginally larger than those at positive velocities, by $0.10,0.03,0.22$, and 0.26 dex for O vi, Nv, C Iv, and Si Iv, respectively. In the region of the sky with excess total column density, these differences are $0.07,0.16,0.25$, and $0.20 \mathrm{dex}$, while in parts of sky away from the excess or deficit region the differences are $0.19,0.05,0.23$, and 0.31 dex. However, in the region with the $\mathrm{O}$ VI deficit, the differences are $-0.15,-0.17$,
0.12 , and 0.15 dex. Since the dispersions in the averages are about $0.20-0.25 \mathrm{dex}$, these differences are generally only about 0.5 times the spread, which according to the $t$-test is barely significant (probability of it happening by chance $2 \%$ ). However, all for four ions the column density at negative velocities appears to be slightly larger than that at positive velocities, so the effect is probably real.

\subsection{Scale Heights}

The issue of the scale heights of the different high-ionization species was addressed by Savage \& Wakker (2009). They combined column density measurements from a number of sources. Data from the Copernicus and FUSE satellites were used to 

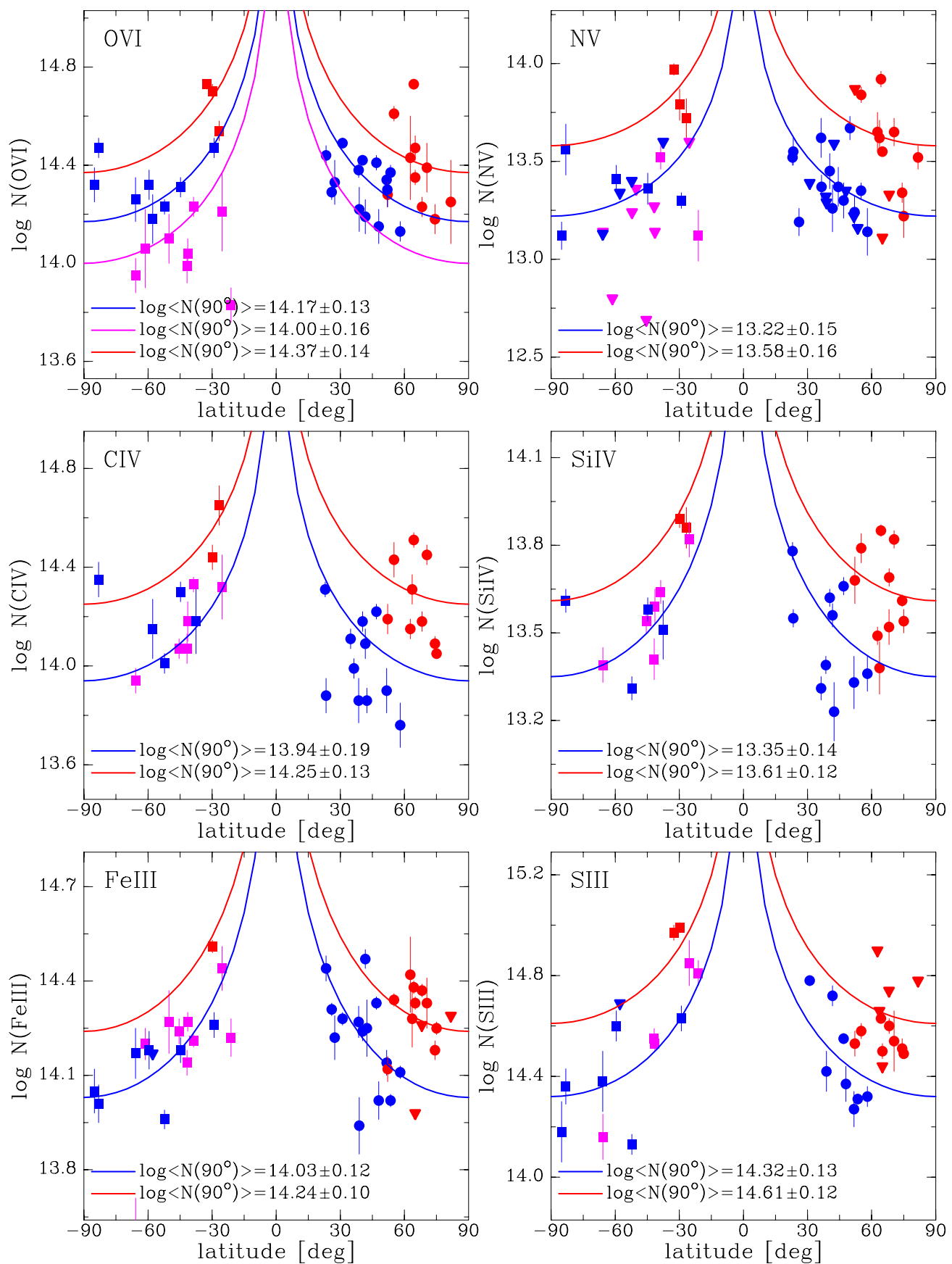

Figure 8. Scatter plot of ionic column densities against Galactic latitude. Symbol types and colors are the same as in Figure 7. In each plot, we also give a curve representing the $N\left(90^{\circ}\right) \sin |b|$ distribution of column densities that would result from a plane-parallel layer, with the values of the corresponding polar column densities given in the lower left corner of each panel.

derive O VI column densities toward stellar and extragalactic targets (using measurements by Jenkins 1978b; Zsargó et al. 2003; Wakker et al. 2003; Bowen et al. 2008). HST spectrographs provided Al III, Si IV, and C IV spectra for a number of extragalactic targets, while IUE was used to measure these ions in stellar spectra and toward Magellanic Cloud stars (Savage et al. 2001a). The total sample included 109 stars and 30 extragalactic objects, all having reliable data.

Using AGNs at $-90^{\circ}<b<45^{\circ}$ (i.e., omitting the region with O vi excess), Savage \& Wakker (2009) derived scale heights of $2.6 \pm 0.6 \mathrm{kpc}$ for $\mathrm{OVI}, 3.6_{-0.8}^{+1.0} \mathrm{kpc}$ for $\mathrm{C} \mathrm{IV}$, and $3.2_{-0.6}^{+1.0}$ for $\mathrm{Si}$ IV. They argued that the slightly smaller O VI scale height compared to CIV and Si IV is consistent with predictions of hybrid coronal gas models in which these ions are produced by collisional ionization in cooling transition temperature gas, but with photoionization from the extragalactic background radiation increasing the amount of C IV and Si IV at high- $z$. They also found that C IV and Si IV are relatively tightly correlated, with an average logarithmic ratio $\mathrm{Si}$ IV $/ \mathrm{C}$ IV $=-0.60 \pm 0.11$ in the thick disk.

With the new set of measurements for $\mathrm{Nv}$, we also tried to apply the methods of Savage \& Wakker (2009) to derive a scale height for $\mathrm{N} \mathrm{v}$, using data from the literature for measurement of $\mathrm{N} v$ toward disk stars (as listed by Savage \& Wakker 2009 and complemented by values from Lehner et al. 2011). However, a fair comparison between the different scale heights requires using the same set of stars and the same selection criteria for all ions. This results in a sample of $\mathrm{N} \mathrm{v}$ measurements that is too 


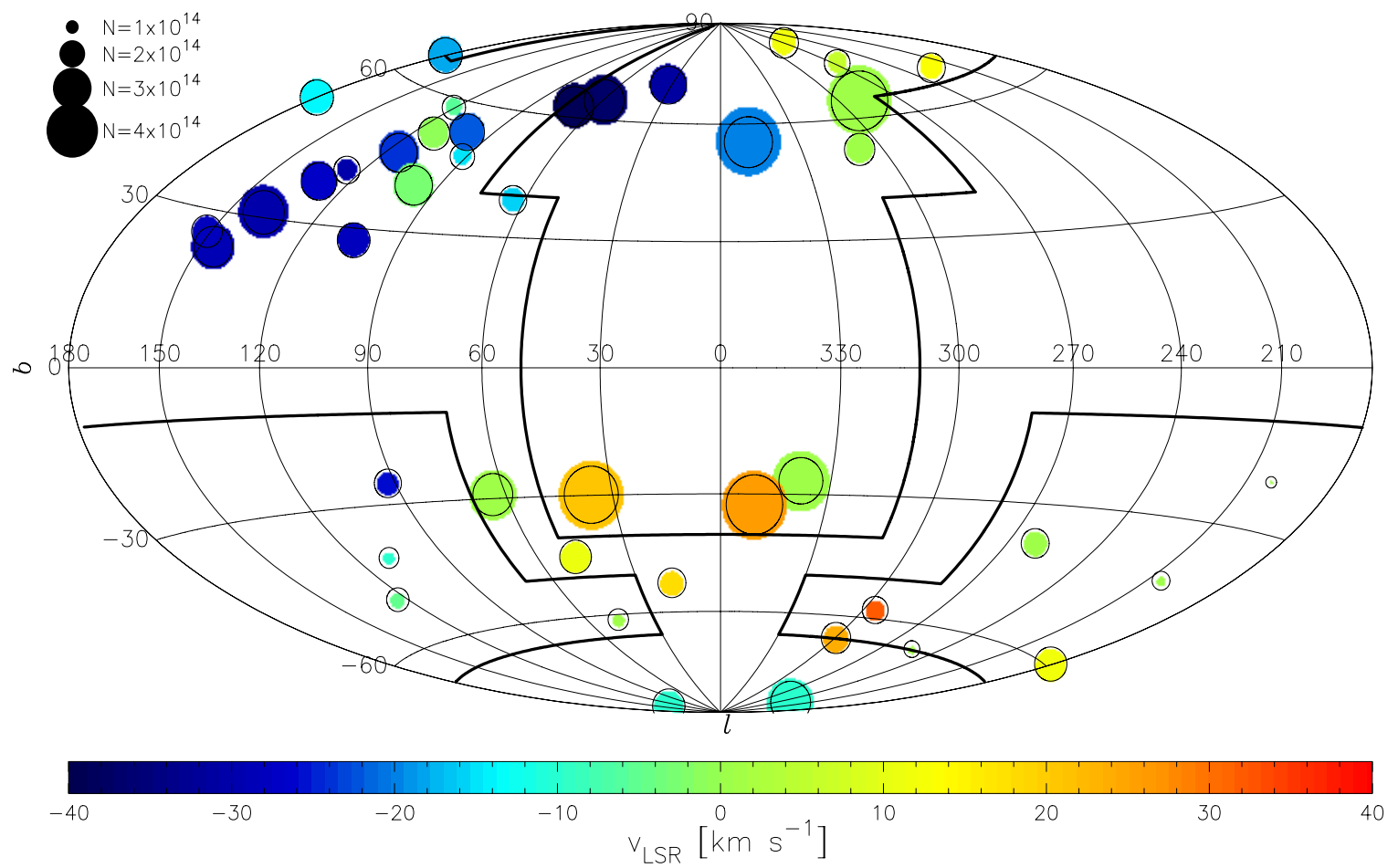

Figure 9. All-sky map of the O vi column density and centroid velocity, in Aitoff projection, centered on the Galactic center. Colors correspond to the centroid velocity of the Galactic O VI absorption, following the scale on the bottom. Symbol diameters are proportional to the value of $N(\mathrm{O}$ vI), following the legend in the upper left corner. The thick lines outline the regions with an excess or deficit in the O vI column density.

small to give a reliable answer. With somewhat less restrictive criteria, the derived scale height for $\mathrm{NV}$ is similar to that of $\mathrm{O}$ VI and C IV. A proper assessment of the $\mathrm{N} v$ scale height will require more data, however.

The fact that both the line widths and the scale heights of C IV and Si IV are comparable and that their centroids are very well aligned is strong evidence in favor of the hypothesis that these ions originate in the same parcels of gas. Comparing to $\mathrm{O}$ VI we find that the scale height of O vi is slightly smaller, while the extent of the $\mathrm{O}$ VI absorption tends to be slightly wider, but the centroids of the $\mathrm{OVI}$ and $\mathrm{CIV} / \mathrm{Si}$ IV are similar. The most likely explanation for these similarities and differences is that the $\mathrm{O}$ VI is associated with the same structures in which $\mathrm{C}$ IV and Si IV originate, but near the plane the O VI-containing gas is slightly more turbulent, while as one goes up, the fraction of $\mathrm{O}$ vI-containing gas decreases.

\subsection{Line Ratio Histograms}

Figure 10 shows the distributions of all ratios between the four highly ionized species, as well as two ratios involving $\mathrm{Fe}$ III, for the absorption components where the derived ratio is flagged as being reliable. The panels on the left side give the distributions of the logarithms of the ratios, the panels on the right show the ratios linearly. The labels give the average ratio and its dispersion, excluding from the calculation some outliers, which are discussed below.

For the most part these distributions are rather narrow. In particular, the histograms of $\log (\mathrm{N}$ v/O vI) and $\log (\mathrm{Si}$ IV/C IV) have a dispersion of only $0.14 \operatorname{dex}( \pm 40 \%)$, with a spread between the highest and lowest ratio of just $0.6 \mathrm{dex}$, or a factor $\sim 4$. The dispersions in the distributions of $\log \left(\mathrm{C}_{\mathrm{IV}} / \mathrm{O} \mathrm{VI}\right)$, $\log (\mathrm{SiIV} / \mathrm{O} \mathrm{VI}), \log (\mathrm{C} \mathrm{IV} / \mathrm{Nv})$, and $\log (\mathrm{Si} \mathrm{IV} / \mathrm{Nv})$ are $\sim 0.23$ dex $(70 \%)$, significantly larger than those in $\log (\mathrm{N} \mathrm{v} /$ $\mathrm{O}$ VI) and $\log (\mathrm{Si} I V / \mathrm{CIV})$. This again supports the notion that
$\mathrm{O}$ vi and $\mathrm{Nv}$ originate together, as do Si IV and C IV, but although the two pairs of ions live in the same structure, they are not fully cospatial. The median error in each individual ratio is about 0.10 dex. Thus, in the case of $\mathrm{N} \mathrm{v/O} \mathrm{VI} \mathrm{and} \mathrm{Si} \mathrm{IV/C} \mathrm{IV,} \mathrm{the}$ observed dispersions in the distributions may reflect the observational uncertainty more than an intrinsic spread. For the other ratios there clearly are variations across the sky.

Previous determinations of these line ratios were presented by Sembach \& Savage (1992), who measured N v, C IV, and Si IV toward 12 high $-z$ stars. They listed an average $\mathrm{C}$ IV $/ \mathrm{N} v$ ratio of $4.6 \pm 2.7$, where we find $5.6 \pm 2.3$, using 24 components. For $\mathrm{C}$ IV/Si IV they found $3.6 \pm 1.3$, compared to our value of $4.2 \pm$ 1.5. Indebetouw \& Shull (2004b) also presented average ionic ratios, giving $\log (\mathrm{N} \mathrm{v} / \mathrm{O} \mathrm{vI})=-0.83 \pm 0.28$ (34 sight lines) and $\log \left(\mathrm{C}\right.$ IV $\left./ \mathrm{O}_{\text {VI }}\right)=-0.28 \pm 0.18$ (11 sight lines $)$, compared to our results of $-0.87 \pm 0.14$ (31 components) and $-0.19 \pm$ 0.21 (44 components) for these ratios, i.e., we find similar values for $\mathrm{C}$ IV/O vi, but a much narrower distribution for $\mathrm{N} \mathrm{v/O} \mathrm{vI,}$ which in large part can be explained as the result of the very different velocity ranges over which the lines were integrated.

\section{IONIZATION MECHANISMS}

The comparison between two line ratios can be a great tool to diagnose the physical conditions in the gas giving rise to the high-ionization absorption. In earlier work the diagnostic value of ionic ratios was often pointed out (e.g., Sembach \& Savage 1992; Spitzer 1996; Savage et al. 1997a), and the observed values of, e.g., $\mathrm{C}$ IV/O vi or C IV/N v were compared with model predictions. However, the diagnostic power of the ratio-ratio diagram (first discussed by Indebetouw \& Shull 2004a; Fox et al. 2004, 2005) was only fully appreciated after FUSE O VI data became available to complement STIS observations of C IV, $\mathrm{N} v$, and Si IV. 

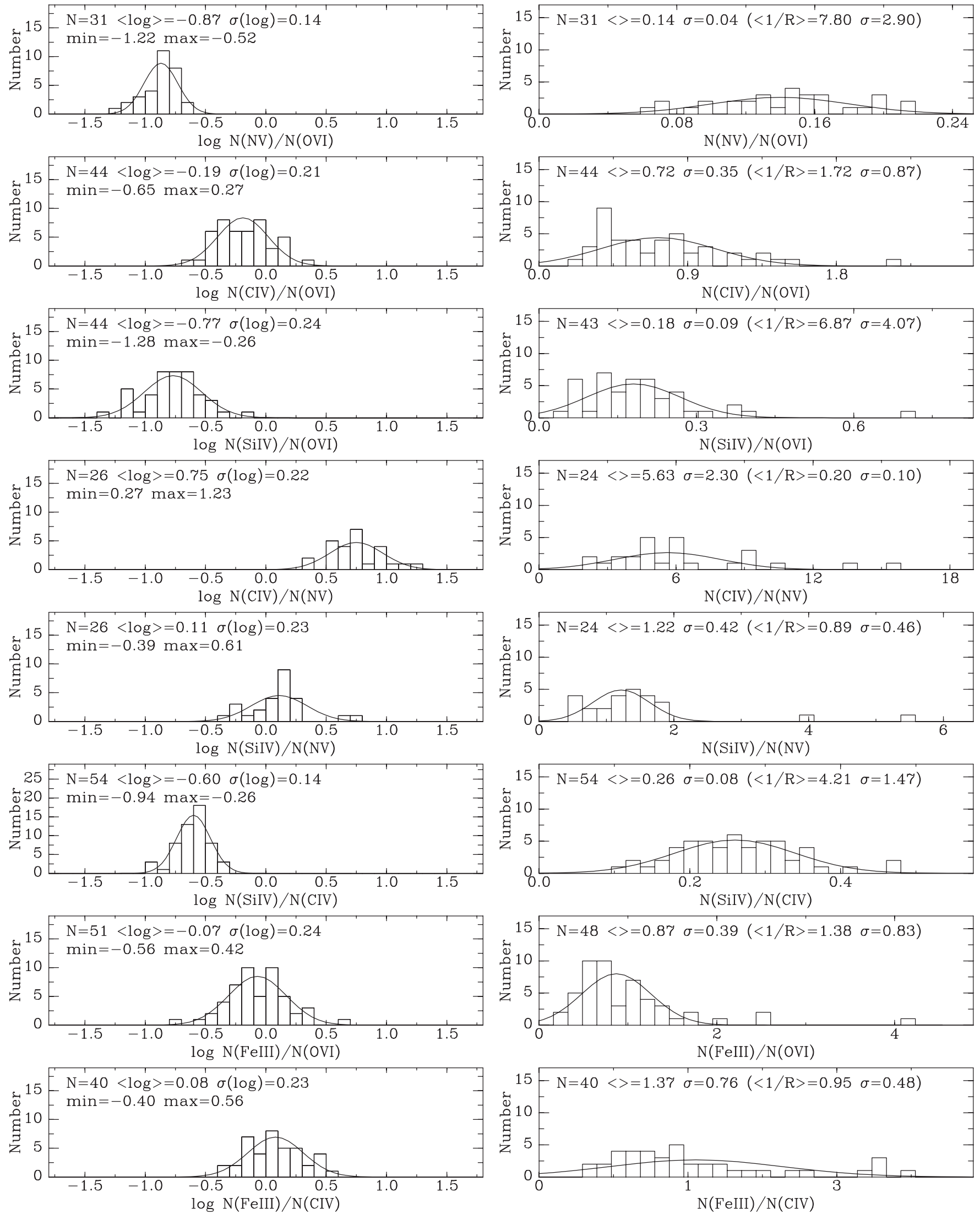

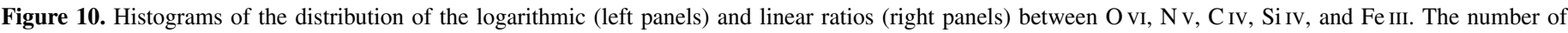

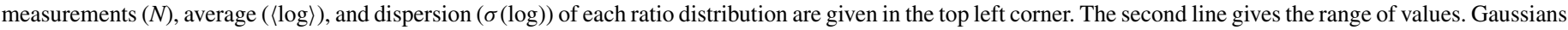

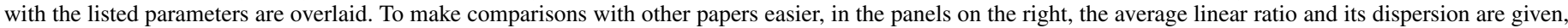

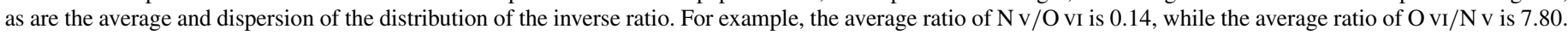


In this section, we summarize the potential ionization mechanisms and compare the resulting predictions for ionic ratios and ionic column densities to the observations. Much of this has been done in one form or another in previous papers. However, unlike previous authors, we describe more explicitly the actual predictions in the different papers in which a given model is calculated, rather using a simplified range of possible values for the ratios. Furthermore, for some processes (collisional ionization equilibrium (CIE), turbulent mixing) revised models were published fairly recently, using updated atomic parameters. We also include three calculations that traditionally have not been part of such an overview: thick disk supernovae (Shelton 1998), static non-equilibrium radiative cooling (Gnat \& Sternberg 2007), and cooling flows (described below). In the case of the last of these, we present this model in a journal paper for the first time, although the predictions were summarized in a conference paper (Shapiro \& Benjamin 1991). Furthermore, we include a new numerical model for turbulent mixing, which includes nonequilibrium ionization (NIE; Kwak \& Shelton 2010).

This set of mechanisms does not exhaust the range of ideas published in the literature. Indebetouw \& Shull (2004a) also list as possibilities evaporating shells, stellar wind bubbles, supernova remnants, and photoionization by white dwarfs. However, some of these appear unlikely because they predict ionic ratios that are very different from the observed values, or because one would expect a small covering factor or large variations in column density across the sky since objects like stellar wind bubbles and white dwarfs have a small volume filling factor.

Historically, different elemental abundances have been used to create the different models. This is because the standard reference values of the solar abundances of most elements (but especially C, N, and O) have varied since 1980, as determinations improved or problems with the interpretation of solar absorption lines were corrected. We therefore adjusted the published predictions of ionic column densities to reflect the most recent values for the solar abundances of $\mathrm{C}, \mathrm{N}, \mathrm{O}$, and Si (Asplund et al. 2009: $\log A(\mathrm{C}, \mathrm{N}, \mathrm{O}, \mathrm{Si})=-3.57,-4.17$, -3.31 , and -4.49 , respectively). However, this adjustment is not a simple scaling, as to first order the O vi column density is independent of metallicity, as the cooling is dominated by the oxygen ions. Thus, a lower oxygen abundance results in less efficient cooling, causing a larger region with transition temperature gas, giving a fixed OVI column density. The column densities of other ions then scale with the change in the ratio of elemental abundances. A different situation prevails in the turbulent mixing model, where the heating up of cold gas is important. In that case the column densities of the highly ionized atoms do scale directly with the elemental abundances.

We compare the models to the data in Figures 11(a)-(d), which plot four combinations of ratios. Figure 11(a) shows $\mathrm{Nv} / \mathrm{OVI}$ against SiIV/C IV, i.e., the ratios for the pairs of ions that seem to originate in the same regions of the ionized structures in the sight line. Figure 11(b) gives N v/O vi against $\mathrm{C}$ IV/O vi which represents a more global comparison through the ionized structure, with the $x$-axis relevant for the hottest gas and the values in the vertical direction influenced by the relative amount of the two different regions in the structure. In Figure 11(c), we show $\mathrm{C}$ IV/O vi against Si IV/CIV, which focuses on the cooler regions. Finally, Figure 11(d) presents $\mathrm{C}$ IV/N v against Si IV/C IV, equivalent to Figure 11(c), but chosen by bearing in mind that COS spectrograph on HST is producing many additional spectra of QSOs that will include data for N v, C IV, and Si IV, but not for O VI.

\subsection{Observed Ionic Ratios}

The filled data points in Figure 11 represent the ratios (or limits, shown by triangles) in the individual components of the Galactic absorption profile. We draw a box around the data points which represents the $\pm 2 \sigma$ range around the average.

To determine whether our measurements for the highly ionized gas in the Milky Way corona are representative, we also looked at the measurements in other environments: the Galactic disk, HVCs, and high-redshift damped Ly $\alpha$ absorbers (DLAs). We discuss each of these below.

For the Galactic disk, we used the measurements of highly ionized atoms toward 38 stars by Lehner et al. (2011), shown by open stars in Figure 11. In most cases O vi, C IV, and Si IV can be measured, while a value or upper limit is given for $\mathrm{N} \mathrm{v}$ in 22 sight lines. Lehner et al. (2011) present a table of the integrated column densities, as well as one for component column densities. However, for the latter the different ions are not explicitly matched up, and a few times the fitted component structure differs for, e.g., C IV versus O VI. In many sight lines the stellar continuum is difficult to define, especially for $\mathrm{N} \mathrm{V}$ and $\mathrm{O}$ vi. Toward many stars the $\mathrm{N} v$ line is also relatively noisy. In other sight lines the C IV and/or Si IV lines are extremely deep and show evidence for saturation, making the derived column densities suspect, and indeed in every one of those cases the $\mathrm{Si}$ IV/C IV ratio is found to lie about 0.3 dex outside the range found at high latitude toward AGNs. In a number of cases the apparent interstellar O vi profile strongly differs from the C IV/Si IV profiles, which may indicate the presence of stellar absorption lines. Reassessing the Lehner et al. (2011) sample, we decided to keep only the 12 sight lines where the stellar continuum is relatively clean and toward which the interstellar absorption lines are not saturated. These are the stars given number $1,2,6,10,11,12,13,14,27,33,35$, and 38 in the Lehner et al. (2011) list. With this restriction of only including measurements for stars with unsaturated and unambiguous absorption, the ratios for these disk sight lines fall completely within the box determined from the extragalactic targets (with the exception of N v/O vi toward HD 99857, which is $1.5 \sigma$ outside the box), indicating that the physical conditions in the transition temperature gas do not change much with height above the Galactic plane.

Another kind of cloud in which highly ionized gas is often detected is the HVCs. Fox et al. $(2004,2005,2010)$ and Collins et al. $(2004,2005)$ analyzed these lines toward the two largest HVCs (complex C and the Magellanic Stream). Sembach et al. (2003) presented measurements of O VI in 84 HVCs seen in sight lines toward extragalactic targets, while Lehner \& Howk (2010) find highly ionized HVCs toward several Galactic stars. Each of these papers shows a small subset of the total available data. In order to look at the ionic ratios in the HVCs, we therefore measured O VI, N v, C IV, and Si IV in each of the sight lines used in this paper. The full results will be presented in a future paper on highly ionized gas in HVCs. We find that detecting $\mathrm{N} \mathrm{v}$ in HVCs is rare, but out of about 25 limits that can be formed for $\mathrm{Nv} / \mathrm{O}$ vi only three fall outside the observed range for Milky Way coronal gas. Similarly, for the about $20 \mathrm{HVCs}$ in which C IV and $\mathrm{O}$ VI are both seen, the ratio falls in the observed range for the Milky Way coronal gas. In the case of Si IV/C IV this is true for all but two of the $\sim 25$ possible ratios. The two exceptions 

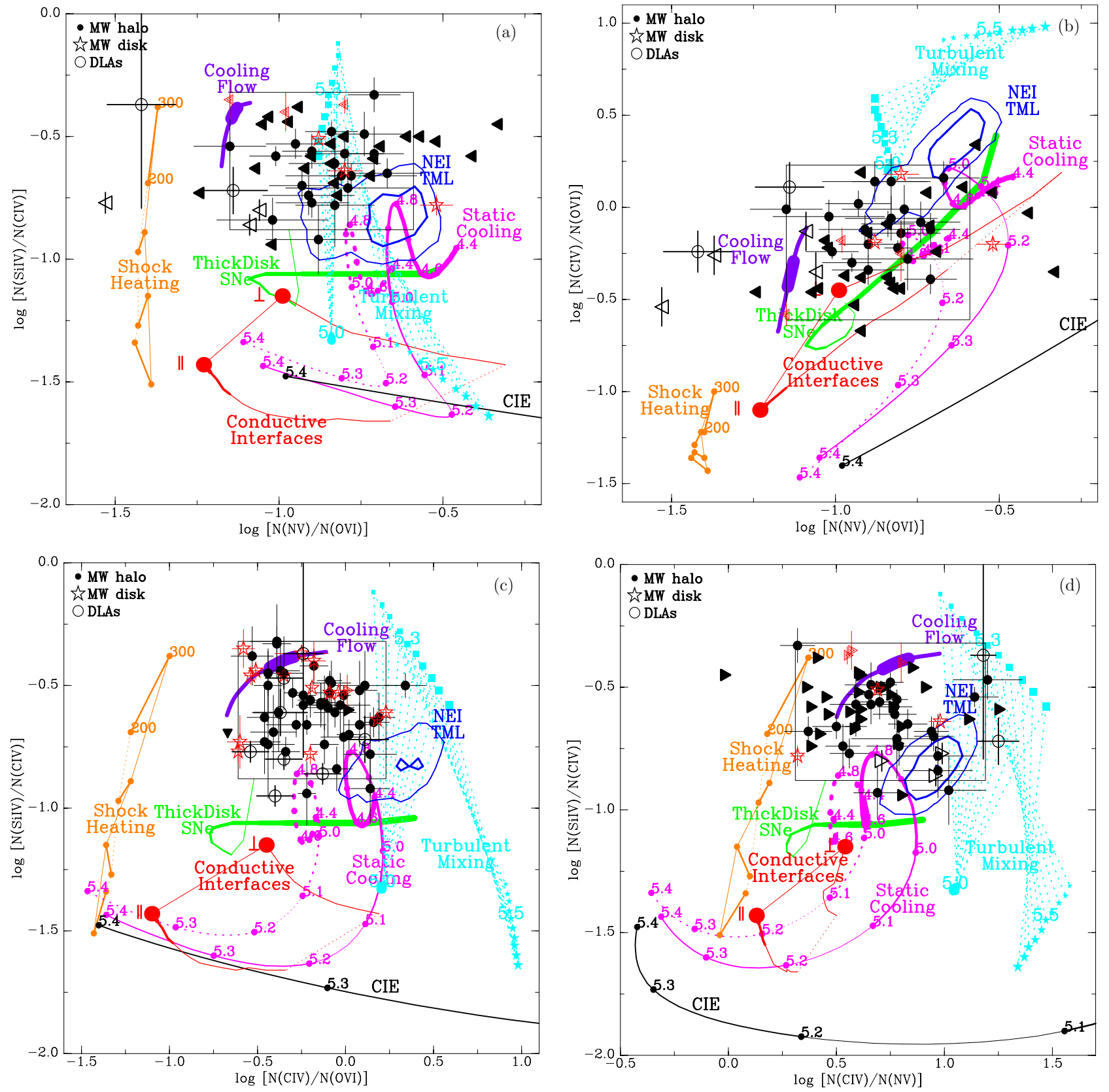

Figure 11. Theoretical ranges and observed values for pairs of ionic ratios. DATA: Filled black circles show ratios in individual components, as determined in Section 3, black triangles show cases with one ratio measured, but the other ratio being an upper or lower limit. A black box outlines the region given by $\langle R\rangle \pm \delta R$, where the average $\langle R\rangle$ and spread $\delta R$ of the ratio distribution can be found in Figure 10. Open red stars and red triangles represent the results of Lehner et al. (2011), limited to their stars numbered $1,2,6,8,9,10,11,12,13,14,23,27,28,32,33,35$, and 38 , i.e., not including stars for which the lines are saturated, have very complex component structure, or difficult to determine stellar continua. Note that this selection leaves just six sight lines with measured values for $\mathrm{N} v$, while in two other sight lines O VI is missing; thus fewer than 12 symbols show up in each individual ratio-ratio plot. Open black circles and triangles represent measurements in DLAs at redshifts 2.0-3.1, as presented by Fox et al. (2007). MODELS: (a) Black line (label: CIE): ratios in collisional ionization equilibrium, as calculated by Gnat \& Sternberg (2007), for temperatures ranging from $\log T=5.4$ to $\log T=4.4$. (b) Magenta lines (label: Static Cooling): non-equilibrium radiative cooling models from Gnat \& Sternberg (2007) for temperatures ranging from $\log T=5.4$ to $\log T=4.4$, with a solid line for solar metallicity and a dotted line for two times solar metallicity; the line gets thicker at lower temperatures, where the gas spends more time; the gas traverses this trajectory in about $3 \times 10^{4} / n_{0}\left(\mathrm{~cm}^{-3}\right) \mathrm{yr}$. In these models the gas is not flowing, and there is no additional heat input or output. (c) Orange lines (label: Shock Heating): Dopita \& Sutherland (1996) shock models, with shock velocities of 200 and $300 \mathrm{~km} \mathrm{~s}^{-1}$ for the no-magnetic-field case marked and connected by a thick line; the other three thick line segments connect the 200 and $300 \mathrm{~km} \mathrm{~s}^{-1}$ case for higher magnetic parameters, $B n^{-3 / 2}=1,2$ and $4 \mu \mathrm{G} \mathrm{cm}^{-3 / 2}$. (d) Red lines (label: Conductive Interfaces): the Borkowski et al. (1990) conductive interface predictions; a "perpendicular" and a "parallel" symbol mark which of the two lines is for perpendicular or parallel magnetic field; the line thickness increases linearly with the age of the interface, $0.1 \mathrm{Myr}$ at the thinnest point, $10 \mathrm{Myr}$ at the other end, which is reached after 1-2 Myr. As a result, the line for this model appears as a quickly traversed thin red line ending in a large dot, where the ratios stabilize. (e) Green line (label: thick disk SN): the Shelton (1998) thick disk supernova model (SNe occurring at heights up to $2 \mathrm{kpc}$ ), giving the evolution of the ratios with time (from 1 to $16 \mathrm{Myr}$ in age), with the line thickness proportional to the amount of time spent at each point. (f) Light blue lines (label: Turbulent Mixing): predictions of the Slavin et al. (1993) semi-analytic turbulent mixing layer model; circles, squares, and stars are shown for entrainment velocities ranging from 25 to $100 \mathrm{~km} \mathrm{~s}^{-1}$ at temperatures of $\log T=5.0,5.3$, and 5.5 , respectively; the symbol size is proportional to the entrainment velocity. (g) Solid blue contours (label: NEI TML): distribution of ratios in the Kwak \& Shelton (2010) turbulent mixing layer models, corresponding to 256 column densities derived by integrating through an interface at $1 \mathrm{Myr}$ intervals for ages between $20 \mathrm{Myr}$ and $80 \mathrm{Myr}$. (h) Dark purple line (label: Cooling Flow): the predictions of the model by Benjamin \& Shapiro that is described in the Appendix. This model is applicable to cooling hot gas flowing through an interface. The range of flow velocities is $20-30 \mathrm{~km} \mathrm{~s}^{-1}$ (thicker part of the line) and 12-42 $\mathrm{km} \mathrm{s}^{-1}$ (thinner part). For these flow velocities the absolute column densities are within 2 and 1 times the observed dispersion around the observed average value. 
are clouds with relatively narrow lines, where it appears likely that photoionization plays a role.

Open circles and triangles in Figure 11 show the measurements of Fox et al. (2007) associated with a number of DLAs at redshifts 2.0-3.1. They found 12 absorption line systems in which at least three of O VI, N v, C IV, and Si IV could be measured. If we use these results and derive ionic ratios, we find that the eight systems for which both $\mathrm{Si}$ IV/C IV and C IV/O VI can be calculated give ratios that fall in exactly the same range as the Milky Way results. N v, however, seems underabundant, with only three detections and five upper limits. In all of these cases $\log \mathrm{N}$ V/O VI is $<-1.1$, which contrasts with the average value of -0.87 seen in the Milky Way.

The metallicities found in the neutral portions of these DLAs (as determined from $\mathrm{S}, \mathrm{Si}$ or $\mathrm{Zn}$ absorption lines) are $[\mathrm{Z} / \mathrm{H}]=$ $-0.85,-0.91$, and -1.62 for the three DLAs in which $\mathrm{N} \mathrm{v}$ is detected, but much lower (between -1.6 and -2.3 ) for the five cases with an $\mathrm{Nv}$ upper limit (Fox et al. 2007). Thus, the non-detections are likely due to a lack of metals. In two of the three DLAs with $\mathrm{NV}$ and $\mathrm{OVI}$ detections, the [N/O] ratio could also be measured directly from $\mathrm{N}_{\mathrm{I}}$ lines, resulting in $[\mathrm{N} / \mathrm{O}]=-1.2$ and -1.1 (Fox et al. 2009). This follows the general trend that nitrogen is underabundant in DLAs (e.g., Petitjean et al. 2008). Thus, the finding that the $\mathrm{N} \mathrm{v} / \mathrm{O}$ vi ratios in DLAs are lower than in the Galactic thick disk can be explained as a differential abundance effect for nitrogen, and is not indicative of any difference in physical conditions.

We note that in nearby dwarf galaxies with metallicity $[\mathrm{Z} / \mathrm{H}]<-1$ times solar, the $\mathrm{N} / \mathrm{O}$ ratio is about 0.8 dex below its value in the Sun (and in the Milky Way; Skillman 1998). This effect can be explained as the result of a difference in the relative amount of $\mathrm{N}$ produced by Type II supernovae (primary $\mathrm{N}$ ), compared to $\mathrm{N}$ formed in intermediate-mass stars (secondary $\mathrm{N}$ ). If the gas sampled by the $z=2-3$ systems was blown out from galaxies at early times, all $\mathrm{N}$ would be primary, and the $\mathrm{N} / \mathrm{O}$ ratio would be subsolar, which can explain the relatively low $\mathrm{N} \mathrm{v/O}$ vi ratios.

Independent of the cause, however, it is clear that $\mathrm{N} v / \mathrm{O}$ vI in the high-redshift DLAs differs from that in the Milky Way. Thus, adapted numerical models are necessary, requiring subsolar $\mathrm{N} / \mathrm{O}$. However, since most of the cooling occurs through the ions of $\mathrm{O}$ and $\mathrm{C}$, it is likely that the effects of using subsolar $\mathrm{N} / \mathrm{O}$ are minimal and that the predicted $\mathrm{N} v$ column densities would scale linearly with N/O.

\subsection{Collisional Ionization Equilibrium (CIE)}

The simplest calculation involves assuming a cloud that has fixed temperature and density, in which every collisional ionization is balanced by a recombination. Sutherland \& Dopita (1993) provided a table for the resulting ion fractions, as did Gnat \& Sternberg (2007). The predictions in the latter paper are about 0.3-0.4 dex higher for N v, C IV, and Si IV at temperatures around $\log T=5.3$, which is caused by changes in atomic parameters (see references in Gnat \& Sternberg 2007). The Si IV fraction differs even more above $\log T=5.4$. On the other hand, the predicted $\mathrm{O}$ VI fraction is rather similar, except above $\log T=6.0$, where the Gnat \& Sternberg (2007) model predicts less O VI. All this results in predictions for the line ratios that can differ by as much as 0.4 dex from the Sutherland \& Dopita (1993) values.

However, we do not expect the gas to be in CIE (and it is not observed to be). First of all, O vi will only exist at temperatures above about $10^{5} \mathrm{~K}$, but then the $\mathrm{Si} \mathrm{IV} / \mathrm{C}$ IV ratio is $\ll-1.5$, i.e., much smaller than what is observed. Moreover, if we apply the CIE ionization fractions to a cloud with $\log N(\mathrm{H})=19$, then $\log N(\mathrm{O}$ vi) can only be $\sim 14.1$ for temperatures above $\log T=$ 5.3 , while $\log N(\mathrm{C}$ IV $)$ is $\sim 13.8$ for temperatures near $\log T \sim$ 4.8 and $\log T \sim 5.2 . \log N(\mathrm{Si} \mathrm{IV})$ only reaches the observed value of 13.2 if $\log T<5.0$, i.e., for temperatures at which the CIE model predicts the presence of $\mathrm{O}$ VI, there is no $\mathrm{C}$ IV and no $\mathrm{Si}$ IV, while at lower temperatures a sufficient amount of $N(\mathrm{C}$ IV $)$ and $N(\mathrm{Si} \mathrm{IV})$ is only present in the absence of $\mathrm{O}$ VI.

\subsection{Static Non-equilibrium Ionization Radiative Cooling}

Edgar \& Chevalier (1986) were the first to predict the ionization properties of gas starting at high temperature $\left(10^{6} \mathrm{~K}\right)$ and cooling down radiatively. In such models, the gas starts out in CIE at a high temperature $\left(5 \times 10^{6} \mathrm{~K}\right.$ in their case $)$, and then is allowed to cool, either isochorically (at constant density) or isobarically (at constant pressure). In these models there are no external sources of heat or photoionization, the gas is not flowing, and it is assumed that the column density is low enough that the gas is optically thin. As most of the cooling is provided by metal-line emission, the metallicity of the gas will determine the timescale of the cooling. At high metallicity, the gas can cool, but highly ionized atoms will recombine too slowly, leading to an overabundance of ions such as $\mathrm{C}$ IV, $\mathrm{N} \mathrm{v}$, and O vi compared to gas in CIE at the same temperature. That is, the ionization is frozen in for a while. The higher the metallicity, the faster the cooling and the larger the discrepancy.

The Edgar \& Chevalier (1986) paper gave predictions for both isochoric and isobaric cooling. For the isobaric case, the values of Si IV/C IV, C IV/O vi, and C IV/Nv are 0.5-1.0 dex below the observed values, while in the isochoric case these ratios are underpredicted by $0.2-0.4$ dex. Although this does not match the observations, the model was influential since it showed that it is possible to get column densities and ionic ratios that are very different from those in CIE.

Gnat \& Sternberg (2007) presented a more detailed set of predicted ionization fractions for a variety of static nonequilibrium models. They provided tables for 10 cases: isochoric and isobaric cooling at metallicities $Z=0.001,0.01,0.1,1$, and 2 times solar. For metallicities $Z<0.5$ solar, the difference with CIE predictions is minimal. Similarly, the difference between the isochoric and isobaric cases is one of degree. In particular, for $Z=0.1$, the isobaric and isochoric predictions differ by less than 0.1 dex, while the $Z=2$ isobaric case is almost identical to the $Z=1$ isochoric case.

Gnat \& Sternberg (2007) also discussed when it would be appropriate to use the isobaric or the isochoric case. They took a spherical cloud which starts out cooling isobarically, and thus contracting as the temperature decreases. This increases the column density. If it becomes larger than $N_{\mathrm{tr}}=1.2 \times$ $10^{19}\left(T / 10^{5}\right)^{2.04} \mathrm{~K}$, the cooling becomes isochoric. With the ionization fractions predicted by the isobaric cooling model, we can derive the implied total hydrogen column density for an $\mathrm{O}$ VI column density of $\log N(\mathrm{O} \mathrm{VI})=14.1$. This value is below $N_{\text {tr }}$ for temperatures $\log T>5.3$, implying that at lower temperatures the appropriate cooling model to use is the isochoric one.

To assess whether an NIE radiative cooling model is realistic, one should not only look at ionic ratios, but also take into account the predicted total ionic column densities, the cooling time, and the integrity of a cloud as it cools and moves. The column densities are within 0.2 dex of the observed averages for temperatures between $\log T=4.3$ and 5.1, if the cloud has solar metallicity or higher and total hydrogen column density 
$\log N(\mathrm{H}) \sim 19$. For $Z=0.1$, however, the cooling is so slow that recombination can keep up and the column densities of $\mathrm{O}$ VI, $\mathrm{N} \mathrm{v}$, and C IV only barely match the observations at $\log T=5.2$, long before any Si IV is formed. The cooling time to go from $T$ $=3 \times 10^{5} \mathrm{~K}$ to $10^{4} \mathrm{~K}$ is about $t=3 \times 10^{4} / n_{0}\left(\mathrm{~cm}^{-3}\right)$ yr for a solar metallicity cloud (where $n_{0}$ is the density of $\mathrm{H}^{+}$; O. Gnat 2010, private communication). Given $\log N(\mathrm{H}) \sim 19$ and a scale height of $3 \mathrm{kpc}$, the $\mathrm{H}^{+}$density at $1 \mathrm{kpc}$ is about $7 \times 10^{-4} \mathrm{~cm}^{-3}$, implying a cooling time of 40 Myr. If the gas were moving at a rate of $10 \mathrm{~km} \mathrm{~s}^{-1}$, it would move over $0.4 \mathrm{kpc}$ during that time, which is likely significant relative to its size, but not so large that the cloud would completely disperse. Thus, we conclude that it is possible to construct a realistic situation in which the NIE radiative cooling model might be applicable.

In Figure 11, the magenta lines show the isochoric nonequilibrium radiative cooling model for the temperature range of $\log T=5.4-4.4$. The solid curve is for solar metallicity, the dotted curve for two times solar metallicity. Clearly, this model can match some of the observed ratios, specifically $\mathrm{C}$ IV/N v and C IV/O vi. However, it underpredicts Si IV/C IV and overpredicts N v/O vi. In Figure 11(d), the pattern of data points suggestively lies on a curve with the same shape as the solar metallicity curve, but with higher $\mathrm{Si}$ IV/C IV ratio. Thus, given the many simplifying assumptions, the predictions of this model are close enough to the observed values that we conclude that non-equilibrium radiative cooling is likely to be an important part of the processes that produce the highly ionized atoms.

\subsection{Radiatively Cooling Gas Flows}

The model described in the previous subsection does not take into account the gas dynamics. Doing so substantially changes the predictions for the relative ionic column densities, even with the same prescription for non-equilibrium cooling. Shapiro \& Benjamin $(1991,1993)$ and Benjamin and Shapiro (see Benjamin's thesis (1994) and Shapiro \& Benjamin 1991, 1993) studied this in more detail. Their model follows the thermal and ionization state of gas in a one-dimensional planar, steady-state flow, including the effects of radiative transfer of photons generated by the hot phase of the gas. This results in a substantial boost for the column densities of C IV and especially $\mathrm{Si}$ IV. Since this model has not been fully described in detail in the literature, we present a summary in the Appendix. We note that the physical origin of the flow remains unspecified in this model-it is a description of the physical structure that results after a cooling front develops in a flow, be it an outflowing wind of hot gas or an inflow.

Here, we compare the predictions of the cooling flow model to the observations. This shows that the predicted column densities of all four ions ( $\mathrm{O}$ vI, N v, C IV, Si IV) are within one or two times the observed spread from their average value (see Section 4.5 and Figure 6) for flow velocities between 20 and 30 and 12 and $42 \mathrm{~km} \mathrm{~s}^{-1}$, respectively. The resulting predictions for the ionic ratios are shown by the thick magenta line in Figure 11. This reveals that this model correctly predicts the observed range of the $\mathrm{C}$ IV/O vi, $\mathrm{C}$ IV/N v, and $\mathrm{Si}$ IV/C IV ratios. However, in general the model predicts values for $N(\mathrm{O} v \mathrm{I})$ that are slightly too high and for $N(\mathrm{Nv})$ that are slightly too low values (see the Appendix), leading to slightly lower $\mathrm{Nv} / \mathrm{O}$ vi ratios than observed (by $\sim 0.4$ dex). This is true both for the average value and for most of the column densities seen in individual components where all four ions are detected. Unlike what is the case for all other models, this model predicts good values for both the observed column densities and the observed column density ratios, suggesting that a cooling flow model including self-photoionization represents the physical conditions in the Galactic lower halo fairly well. It is likely that only relatively minor adaptations may be needed to make the predictions for $\mathrm{O}$ VI and $\mathrm{N} v$ fit the observations even better.

\subsection{Shock Ionization}

Shock fronts passing through the gas can create transition temperature gas. A grid of low-density shock models was presented by Dopita \& Sutherland (1996), for shock velocities of $200,300,400$, and $500 \mathrm{~km} \mathrm{~s}^{-1}$, magnetic parameter $B n^{-3 / 2}=$ $0,1,2$, and $4 \mu \mathrm{G} \mathrm{cm} \mathrm{cm}^{-3 / 2}$, and solar abundances. In the Milky Way corona, shock velocities of $400 \mathrm{~km} \mathrm{~s}^{-1}$ are not expected, so we compare data and model only for 200 and $300 \mathrm{~km} \mathrm{~s}^{-1}$ shocks. In these models the ionization of the ions is determined, taking into account non-equilibrium conditions. In these models the logarithm of the $\mathrm{O}$ VI column density is predicted to be 14.32-14.35, while $\log N(\mathrm{Nv})$ spans the range 12.85-12.95. The predictions for $\log N(\mathrm{C}$ IV $)$ and $\log N(\mathrm{Si}$ IV $)$ are much more varied, ranging from 12.8 to 13.0 and 11.3 to 12.8 , respectively, with higher column densities occurring for higher shock velocities and stronger magnetic fields. Thus, shock models predict $\mathrm{O}$ VI and $\mathrm{N} v$ column densities that are a factor of 1.8 and 2.4 larger than the observed average, while the column densities of C IV and Si IV are underpredicted by an order of magnitude. Thus, in the ratio-ratio plots shown in Figure 11, the predictions for the shock models (shown as orange lines) lie far away from the observed values. Therefore, shocks appear to be an unlikely explanation for the observed highly ionized atoms in the Galactic corona.

\subsection{Conductive Interfaces}

If a reservoir of hot gas comes into contact with a cool cloud, electron collisions may conduct heat between the two media, and transition temperature ions can be produced in the conduction front as the cooler gas evaporates and the hotter gas condenses. This process was modeled by Borkowski et al. (1990) and Gnat et al. (2010). In the first of these two papers predicted column densities are shown for several ions as function of time and as function of the angle between the magnetic field and the conduction front. The magnetic field is important because the thermal conductivity of the plasma is far greater along the magnetic field lines than across them.

In the Borkowski et al. (1990) model, the C IV column density stabilizes at more or less constant values (less than $0.3 \mathrm{dex}$ variation) after about $10^{4} \mathrm{yr}$. This is the case for $\mathrm{N} \mathrm{v}$ after $10^{5} \mathrm{yr}$ and for $\mathrm{O}$ vi after about $2 \times 10^{5} \mathrm{yr}$. At earlier times the evolution in the column densities (and thus the ionic ratios) is rapid. Thus, in a ratio-ratio plot, the range of ratios that this model can accommodate varies with the age of the youngest interface that is included. Fox et al. $(2004,2005)$ chose $10^{5} \mathrm{yr}$ as the age of their youngest interface. However, between $10^{5}$ and $2 \times 10^{5} \mathrm{yr}$, the O VI column density has not yet stabilized, and $N(\mathrm{O} \mathrm{VI})$ increases by 0.27 dex. On the other hand, $N(\mathrm{~N} v)$ and $N(\mathrm{Si}$ IV $)$ are constant and $N(\mathrm{C}$ IV) decreases by 0.07 dex. Thus, the $\mathrm{C}$ IV/O vi ratio decreases by $0.34 \mathrm{dex}$ as the interface ages from $10^{5}$ to $2 \times 10^{5} \mathrm{yr}$. It should be noted that these interfaces mature relatively quickly. The ratios traverse the track in a few $10^{5} \mathrm{yr}$, but then they stay constant for the next several $10^{6} \mathrm{yr}$. Thus, for over $90 \%$ of the lifetime of the interface this model predicts a unique value of the ionic ratios. 
Gnat et al. (2010) recently revisited the conductive interface model, exploring a greater range in the parameter space characterizing the interface. They present column densities for mature interfaces in which the surrounding medium has a temperature between $10^{6}$ and $10^{7} \mathrm{~K}$, and in which the pressure ranges from 0.1 to $50 \mathrm{~K} \mathrm{~cm}^{-3}$. They further include photoionization by the extragalactic radiation field, though not magnetic fields. However, unlike Borkowski et al. (1990) they do not tabulate the time evolution of the column densities. Comparing their predicted ionic ratios to those of Borkowski et al. (1990), we find that if the pressure $P / k$ is $>5 \mathrm{~K} \mathrm{~cm}^{-3}$, all of the predicted ratios cluster within 0.2 dex of those found by Borkowski et al. (1990) models for the case of a perpendicular magnetic field. Thus, expanding the parameter space does not substantially change the nature of the predictions, and we therefore do not explicitly show the results of the Gnat et al. (2010) predictions in Figure 11.

In Figure 11, we show with red lines the range of ratios for times $>2 \times 10^{5} \mathrm{yr}$, with one line for the case of a perpendicular magnetic field and one line for a parallel field. We connect the start and end points of these lines to form a box that encloses the full range of possible predictions. The line thickness increases with time. This shows that after about 1-2 Myr the ionic ratios stabilize at a fixed value. Thus, unless one considers a very young interface, this model predicts just a single set of ionic ratios. For the case of the perpendicular magnetic field, the predicted final ratio for $\mathrm{NV} / \mathrm{O}$ VI and $\mathrm{C}$ IV $/ \mathrm{O}_{\mathrm{VI}}$ lies toward the lower end of the observed values, while $\mathrm{CIV} / \mathrm{NV}$ is close to the average. However, Si IV/C IV is underpredicted by about 0.5 dex, and all predictions for the case of a parallel magnetic field differ by about 0.5 dex from the observations. The fact that Gnat et al. (2010) added some photoionization to their models, but produce the same predictions for the line ratios implies that adding photoionization will not help to increase the relative amount of Si IV.

An additional problem with these models is that each interface produces a maximum column density of about $\log N(\mathrm{O}$ VI $)=$ $12.8, \log N(\mathrm{~N} \mathrm{v})=12.1, \log N(\mathrm{C}$ IV $)=12.5$, and $\log N(\mathrm{Si}$ IV $)=$ 11.1. This is in the case with a magnetic field that is perpendicular to the conduction front, and for an initial temperature of the hot phase of $7.5 \times 10^{5} \mathrm{~K}$. For a parallel field these values are 5-10 times smaller, but increasing the temperature of the hot phase to $10^{6} \mathrm{~K}$ increases them by a factor $\sim 2$. Thus, the Borkowski et al. (1990) model can be used to predict the column density ratios, but it cannot explain the observed total column densities. That requires assuming that a sight line intersects many interfaces. Assuming an average angle of $45^{\circ}$ between the magnetic field and the interface (reducing the average column density by a factor two), but also assuming that $10^{6} \mathrm{~K}$ is more typical for the hot gas than $7.5 \times 10^{5} \mathrm{~K}$ (increasing the average column density by a factor two), between 5 and 50 interfaces would be required to build up the observed total column densities. As each interface has a thickness on the order of $10 \mathrm{pc}$, the interfaces would have to occupy a total path length on the order of $100 \mathrm{pc}$, which is easily accommodated within the several kiloparsec scale height of the highly ionized atoms.

To summarize: (1) the conductive interface model strongly underpredicts $N(\mathrm{Si} \mathrm{IV}),(2)$ the time spent as a mature interface with constant ionic ratios is a factor 10 longer than the time spent along the tracks, (3) for a mature interface the predicted $\mathrm{Nv} / \mathrm{O}$ vi and $\mathrm{C}$ IV/O vi ratios are relatively low, and (4) it is difficult to build up the total column densities that are seen, with each interface only contributing a small fraction of the total. We thus conclude that conductive interface physics is not the most important process producing the highly ionized atoms in the Galactic corona. We note that this conclusion is consistent with the Bowen et al. (2008) result that the O VI absorbers in the disk exhibit a broad range of column densities, with the more strongly absorbing clouds having a lower space density than the weakly absorbing clouds. The latter conclusion follows from the fact that while $N(\mathrm{O} v \mathrm{v})$ correlates with distance, the dispersion in $N(\mathrm{O} \mathrm{VI})$ does not decrease as the detected clouds get more distant.

\subsection{Thick Disk Supernovae}

Another model that we consider here is that of Shelton (1998), who analyzed the effect that old Type Ia supernova remnants above the Galactic plane might have on the state of the thick disk gas. She predicted the time evolution of the column densities of $\mathrm{O}$ vi, $\mathrm{N} \mathrm{v}$, and $\mathrm{C}$ IV (though not of Si IV) inside a supernova bubble for an explosion with energy $0.5 \times 10^{51}$ erg expanding into a medium with ambient density $0.01 \mathrm{~cm}^{-3}$ and ambient nonthermal pressure $1800 \mathrm{~K} \mathrm{~cm}^{-3}$. We were separately provided the predictions for the Si IV ion, so that we could properly compare this model to the observations.

In this model, the $\mathrm{O}$ vi column density decreases from an early value of $\log N(\mathrm{O}$ vI $)=14.2$ at $0.1 \mathrm{Myr}$ to about 13.6 at $0.5 \mathrm{Myr}$, then slowly rises to 13.7 at $10 \mathrm{Myr}$, falls to 13.2 at $15 \mathrm{Myr}$ and then it disappears. The other ions behave similarly, plateauing at $\sim 12.8,13.2$, and 12.0 dex for $\mathrm{N} \mathrm{v}, \mathrm{C}$ IV, and Si IV, respectively. $\mathrm{N} v / \mathrm{O}$ VI is about -1.0 up to an age of $10^{7} \mathrm{yr}$, then decreases to $-0.5 \mathrm{dex}$ at $1.5 \times 10^{7} \mathrm{yr}$. On the other hand, in the early stages, $\mathrm{Si}$ IV/C IV first dips from $\sim-1.5$ to $\sim-0.7 \mathrm{dex}$, then reaches a value of $\sim-1.1$ dex after $2 \times 10^{6} \mathrm{yr}$, where it remains throughout.

As can be seen from the green line Figure 11(b), this model predicts values for $\mathrm{NV} / \mathrm{O}$ VI that span the range of observed values. However, if the supernovae have random ages, there should be more ratios near -1.1 (ages $<10 \mathrm{Myr}$ ) than at higher values (ratio slowly increasing until an age of $16 \mathrm{Myr}$ ). Further, this model underpredicts $\mathrm{Si}$ IV/C IV by about $0.5 \mathrm{dex}$ and the absolute values of the column densities during the plateau phase are about $0.4,0.5,0.6$, and 1.2 dex too low for O VI, $\mathrm{N} \mathrm{v,} \mathrm{C} \mathrm{IV,}$ and $\mathrm{Si}$ IV, respectively. The latter problem can be accommodated if between two and three remnants are intersected in each sight line. Since Shelton (1998) derives that the sky coverage fraction due to thick disk supernovae is $30 \%-60 \%$, this implies that the predicted column density is a factor $\sim 3$ to 5 too low to account for the observed column densities.

In summary, this model predicts the correct range of $\mathrm{N} \mathrm{v} / \mathrm{O} \mathrm{VI}$ ratios, but the $\mathrm{Si}$ IV/C IV ratio is underpredicted by $0.5 \mathrm{dex}$ and it predicts a lopsided distribution of $\mathrm{N} \mathrm{v} / \mathrm{O}$ vi ratios. Furthermore, the implied supernovae rate is a factor of three to five too high. Thus, halo supernovae may contribute to the observed quantities of highly ionized atoms, but they cannot be the whole story.

\subsection{Turbulent Mixing Layers}

Conductive heat transfer is not the only process that can take place at the interface between hot and cold gas. Relative sideways motions can cause Kelvin-Helmholtz instabilities that mix the two, creating gas at transition temperatures. This model was proposed by Begelman \& Fabian (1990), and developed by Slavin et al. (1993), Esquivel et al. (2006), and Kwak $\&$ Shelton (2010). These papers present predictions for ionic column densities. 
Slavin et al. (1993) presented predictions for entrainment velocities between 25 and $100 \mathrm{~km} \mathrm{~s}^{-1}$, at temperatures of $\log$ $T=5.0,5.3$, and 5.5, and for two abundance cases-solar abundances and gas in which half of the $\mathrm{C}, \mathrm{N}$, and $\mathrm{O}$ (and $90 \%$ of the $\mathrm{Si}$ ) are on dust grains, for a total of 12 different predictions. In these models the mechanical aspects of the mixing were treated analytically (based on a simplified picture of how the mixing occurs), while the ionic column densities were then predicted using NIE balance calculations including self-photoionization. We show the solar-abundance results of Slavin et al. (1993) in Figure 11 (light blue points and lines).

Since they showed that the change of ionic ratios with entrainment velocity is almost linear, for a given temperature we interpolate the ratios at 25 and $100 \mathrm{~km} \mathrm{~s}^{-1}$ to form a series of points in the ratio-ratio diagram, increasing in size with entrainment velocity. Points with $\log T=5.0$ are shown by closed circles, points at $\log T=5.3$ by closed squares, and points at $\log T=5.5$ by closed stars. High Si IV/C IV occurs at $\log T=5.3$, while high $\mathrm{N} v / \mathrm{O}$ vi occurs at $\log T=5.5$. We then use dotted lines to connect the values for the three different temperatures at the same entrainment velocity. This defines a region in the ratio-ratio diagnostic plot for the predictions of the Slavin et al. (1993) turbulent mixing model. Changing the abundance pattern (i.e., assuming differential depletion onto dust) shifts these points somewhat, but by less than 0.2 dex for $\mathrm{O}$ VI, $\mathrm{N} \mathrm{v}$, and C IV, although Si IV is strongly decreased.

In the Slavin et al. (1993) model, the O vi column density in each interface was only about $10^{11}-10^{12} \mathrm{~cm}^{-2}$, implying the need for more than 100 interfaces to build up the total observed column density of $10^{13}-10^{14} \mathrm{~cm}^{-2}$. De Avillez \& Breitschwerdt (2005) show that this may indeed be possible. They took a hydrodynamical model of the ISM and calculated the density of $\mathrm{O}$ VI as implied by radiative cooling (assuming local CIE in each grid cell). The $\mathrm{O}$ VI originates in the interiors of regions created by supernovae, as well as the result of local cooling promoted by turbulent mixing. They found that on average each sight line out to $1 \mathrm{kpc}$ contains many structures that contribute a few $10^{12} \mathrm{~cm}^{-2}$ of $\mathrm{O} \mathrm{VI}$, and that the total is about $10^{14} \mathrm{~cm}^{-2}$. Although the assumption of local CIE is probably false (given that this predicts the wrong ionic ratios), this shows it could in principle be possible to build up the total observed $\mathrm{O}$ VI column density from many different small structures in the sight line.

The study by Esquivel et al. (2006) addressed the effects of magnetic fields, which mainly result in a reduction of the spread in the predicted ratios for a given flow velocity and density choice. Since Esquivel et al. (2006) did not give a table of predictions, and since Figures 8 and 9 in Esquivel et al. (2006) show that their results are similar to those of Slavin et al. (1993), we do not include this model in Figure 11.

Kwak \& Shelton (2010) revisited the turbulent mixing model, making a two-dimensional hydrodynamical calculation, while following the ionization using non-equilibrium physics (although they still used the CIE value for the cooling function at each temperature). Their base model ("A") uses a $100 \times$ $300 \mathrm{pc}$ box, with hot gas density and temperature $10^{-4} \mathrm{~cm}^{-3}$ and $10^{6} \mathrm{~K}$ and cool gas density and temperature $0.1 \mathrm{~cm}^{-3}$ and $10^{3} \mathrm{~K}$, and an entrainment velocity of $100 \mathrm{~km} \mathrm{~s}^{-1}$. In their paper, they show the time evolution of the column densities of $\mathrm{O} V \mathrm{VI}$, $\mathrm{N} \mathrm{v}$, and $\mathrm{CIV}$, and tabulate the time and sight line averages for these three ions (which are $\log N(\mathrm{O}$ vI $)=12.8, \log N(\mathrm{~N} \mathrm{v})=$ $12.2, \log N(\mathrm{C}$ IV $)=13.1)$, as well as the ionic ratios. Although Kwak \& Shelton (2010) did not publish their predictions for
Si IV, they included it in their calculations, and we obtained the $\mathrm{Si}$ IV values from them.

For each time step, their model produces a two-dimensional map, with 256 directions along which a column density can be derived for each ion. We obtained each of these values, once every Myr for an 80 Myr period. Since it takes about $20 \mathrm{Myr}$ for the effects of initial conditions to disappear, we used the results between 20 and 80 Myr to create a contour plot showing how often a given ratio occurs. The resulting contours are shown in blue in Figure 11. Clearly, this model gets the ratios almost correct, with on average $\mathrm{N} v / \mathrm{O}$ vi about 0.3 dex too high (observed average -0.87 , model average -0.56$), \mathrm{C}$ IV $/ \mathrm{O}$ VI about 0.6 dex too high $(-0.21$ observed average, 0.39 predicted average), $\mathrm{C}$ IV $/ \mathrm{N}$ v about 0.2 dex too high $(0.75$ versus 0.94$)$, and $\mathrm{Si}$ IV/C IV about 0.2 dex too low ( -0.60 versus -0.83$)$. Some of these differences are similar to the mismatches for other models, but for the turbulent mixing model none of the differences is larger than 0.6 dex, unlike what is the case for any other model. The curve showing the evolution of the ratios during static non-equilibrium cooling crosses the turbulent mixing contours, suggesting that in the turbulent mixing model the cooling is not highly affected by the motions of the gas. In this model, the $\mathrm{O}$ VI column density through each $\sim 100 \mathrm{pc}$ thick layer is about log $N(\mathrm{O}$ VI $)=12.8$, so that about 10-20 layers are needed to make up the observed total. These would take up about $1 \mathrm{kpc}$ of the sight lines, which is a substantial, but possible, percentage for gas with a scale height of $3 \mathrm{kpc}$.

We also compared the predictions of the Kwak \& Shelton (2010) model A to those of their models D and F. They already showed that their results are not very sensitive to the parameters (spatial resolution, grid size, entrainment velocity, initial perturbation amplitude, temperature of the hot gas), but that there are some differences. To be precise, model D (entrainment velocity $50 \mathrm{~km} \mathrm{~s}^{-1}$ instead of $100 \mathrm{~km} \mathrm{~s}^{-1}$ ) results in relatively more $\mathrm{Si} I V$ and $\mathrm{C}$ IV, improving the correspondence with the observed values for SiIV/C IV, but it makes the predictions for $\mathrm{C}$ IV /O vi and C IV/N v slightly worse. The same effect, but stronger, happens in model $\mathrm{F}$ (temperature of the hot gas $3 \times 10^{6} \mathrm{~K}$ instead of $10^{6} \mathrm{~K}$ ). Their model A appears to give the best fit. We conclude that turbulent mixing layers may play an important role in explaining the highly ionized ions in the Galactic corona.

\section{DISCUSSION}

We showed in Section 4.2 that the highly ionized gas in the Galactic corona usually is seen at velocities $30-100 \mathrm{~km} \mathrm{~s}^{-1}$ more negative or more positive than can easily be explained by differential galactic rotation. In Sections 4.3, 4.4, 4.5, and 4.6, we analyzed the widths of the absorption lines, the signature of Galactic rotation, the column densities, and the relation between these quantities and location on the sky, respectively. We found that the widths (FWHM) of individual absorption lines range from $\sim 25 \mathrm{~km} \mathrm{~s}^{-1}$ to $\sim 120 \mathrm{~km} \mathrm{~s}^{-1}$. On average the widths of $\mathrm{OVI}$ and $\mathrm{NV}$ absorption lines are similar, as are those of C IV and Si IV, while the average of the latter is slightly, but significantly, narrower $\left(42 \mathrm{~km} \mathrm{~s}^{-1}\right.$ versus $\left.60 \mathrm{~km} \mathrm{~s}^{-1}\right)$. The line width is always much larger than the maximum contribution from thermal motions $\left(\sim 20 \mathrm{~km} \mathrm{~s}^{-1}\right)$, so most of the line width must be non-thermal. Therefore, the four ions cannot be completely cospatial. On the other hand, the widths of $\mathrm{O}$ vI/N v versus $\mathrm{C}$ IV/Si IV correlate very well. That is, in sight lines where $\mathrm{O}$ VI and $\mathrm{N} v$ are wide, $\mathrm{C}$ IV and $\mathrm{Si}$ IV are also wide. Furthermore, there appears to be no difference in the centroids 
of the absorption lines of these ions. Thus, although they do not originate in the same parcels of gas, they must originate in a single structure, in which non-thermal motions are larger in the region where the hotter gas occurs.

There appears to be no relation between galactic latitude and the extent of the absorption, which implies that the velocities of individual gas parcels in the sight line are randomly oriented. If, for instance, the velocities were mostly vertical, projection effects would lead to a relation between the extent of the absorption and latitude, with wider lines at higher latitude. The large spread of velocities in each direction makes the effect of galactic rotation difficult to discern, but we can show that it is responsible for shifting the centroids of the profiles by up to $30 \mathrm{~km} \mathrm{~s}^{-1}$ from zero. We looked for evidence of a vertical gradient in the rotation velocity, by comparing the expected centroids to the observed values. Taking into account that the observed scale height of the gas is about $3 \mathrm{kpc}$, we find that a corotating corona best explains the centroids. We further find that although the distribution of the highly ionized gas is by no means uniform, the variations across the sky are less than an order of magnitude, with no directions toward which these ions are not seen. Much of the variation can be explained as a latitude effect, with a larger path length through the layer at low latitudes. However, over most of the outer Galaxy below the Galactic plane there is a slight ( 0.2 dex, factor 1.6) deficit in $N(\mathrm{O}$ VI $)$, while in the inner Galaxy and over the Galactic north pole there is a slight excess $(0.2-0.4$ dex, factor $\sim 2)$ in the column density of all ions. A description in terms of a planeparallel layer in which the column density changes as $N \sin |b|$ works fairly well. The dispersion around $N \sin |b|$ is then only about $0.15-0.20$ dex (a factor $\sim 1.5$ ). Thus, the highly ionized atoms appear to occur in a somewhat irregular thick layer with scale height $3 \mathrm{kpc}$, which rotates along with the Milky Way, and in which gas parcels have random velocities that are on the order of $\pm 60 \mathrm{~km} \mathrm{~s}^{-1}$.

For a given absorption component and ion, the distribution of column densities is relatively narrow. The range in column densities is no more than a factor of 10 , with a dispersion of a factor of about 2, i.e., for $70 \%$ of the sight lines the observed ionic column density falls within a factor of two of the average. This is compatible with a situation in which the transition temperature gas originates in just a few (three to five) individual structures in each sight line, in each of which the column density is on the order of $1 / 3-1 / 2$ the average observed value. The distribution of column density ratios is even narrower, with the ratios $\mathrm{N} \mathrm{v/O} \mathrm{VI} \mathrm{and} \mathrm{Si}$ IV/C IV having a dispersion of only 0.14 dex (a factor of 1.3 either way). This suggests that in each individual structure the same process is responsible for producing the transition temperature gas everywhere. The fact that the distribution of the $\mathrm{C}$ IV/O VI and $\mathrm{C}$ IV $/ \mathrm{N}$ V ratios is slightly wider 0.23 dex (a factor of 1.7) again indicates that although these ions all come from a single structure, the ions that should occur at somewhat higher temperature are not fully cospatial with the ions that are more prevalent at lower temperatures.

From the ratio-ratio plots shown in Figure 11, we conclude that the physical process(es) that generate the highly ionized gas in the Milky Way's corona must include NIE and involve radiative cooling. In fact, the gas must be very far from CIE, since in CIE OVI cannot coexist with Si IV, and there is no single temperature that simultaneously explains the observed $\mathrm{Nv} / \mathrm{O}$ vi and $\mathrm{CIV} / \mathrm{Nv}$ ratios. Furthermore, for temperatures near $10^{5} \mathrm{~K}$ all ionic ratios differ by at least a factor of 10 from the observed values. Predictions based on shock heating strongly overpredict the amount of $\mathrm{O}$ VI relative to $\mathrm{Nv}$ and $\mathrm{C}$ IV, and even the amount of $\mathrm{N} v$ relative to $\mathrm{C}$ IV. A model in which the transfer of heat occurs purely through electron conduction strongly underpredicts the $\mathrm{Si}$ IV /C IV ratio by a factor of 5-10, and underpredicts N V/O VI and C IV/O VI, although it gets $\mathrm{N} \mathrm{v/O} \mathrm{vi} \mathrm{correct,} \mathrm{but} \mathrm{only} \mathrm{when} \mathrm{the} \mathrm{magnetic} \mathrm{field} \mathrm{is} \mathrm{perpen-}$ dicular to the interface. For a non-perpendicular magnetic field, the predictions of the conductive interface model are rather different from the observations. This model does seem to work reasonably well if one only considers the $\mathrm{Nv} / \mathrm{O}$ vi versus $\mathrm{C}$ IV/O vi ratio-ratio plot. However, compared to the square box used by Fox et al. (2004, 2005), a more detailed look at the actual predictions shows that they fall in a strip parallel to but below the observations (see Figure 11(b)).

We explored four different models that include nonequilibrium radiative cooling, including static cooling, the flow of gas through a cooling front, thick disk supernovae, and turbulent mixing. The original turbulent mixing model of Slavin et al. (1993) predicts much larger ranges for the ratios than is observed, as well as a factor one to two more $\mathrm{O}$ vi than the newer numerical simulations of Kwak \& Shelton (2010). Except for the static case, in these models the O VI, N v, C IV, and Si IV do not coexist in the same gas parcels, but they are present in a related way in different parts of the same structure, as is indeed indicated by the observations. Since the predicted column densities are based on an integral through these structures, the predictions account for this effect.

The thick disk supernova model underpredicts Si IV/C IV by about $0.5 \mathrm{dex}$, and predicts a distribution of ratios for $\mathrm{N}$ V/O VI and C IV/O VI that is much more strongly weighted toward lower values than what is observed. For the remaining three models, the predicted range of ratios differs by up to about 0.3 dex from the observations, with static radiative cooling and turbulent mixing predicting a value for $\mathrm{Nv} / \mathrm{O} \mathrm{VI}$, $\mathrm{Nv} / \mathrm{C}$ IV that is too high by about $0.25 \mathrm{dex}$ and $\mathrm{C}$ IV/O VI too high by $0.5 \mathrm{dex}$. At the same time, $\mathrm{Si}$ IV/C IV is too low by about 0.2 dex. That is, for a given amount of $\mathrm{O}$ vi, these models (on average) predict a factor of 1.8 too much $\mathrm{N} \mathrm{v}$, a factor of 3 too much C IV, and a factor of 1.7 too much Si IV. The cooling flow model (with self-photoionization included) predicts the correct range of ratios for $\mathrm{CIV} / \mathrm{O}$ VI and for $\mathrm{Si} I V / \mathrm{C}_{\mathrm{IV}}$, but it underpredicts the amount of $\mathrm{O}$ VI relative to $\mathrm{C}$ IV and $\mathrm{N} v$. Thus, none of the NIE radiative cooling models can quite get all four ions correct at the same time. However, compared to models having equilibrium ionization and no radiative cooling, the former do a much better job.

\section{SUMMARY}

We have analyzed the absorption from highly ionized atoms through the complete Milky Way thick disk and halo as seen in 58 sight lines toward extragalactic targets. These ions are O VI, $\mathrm{N}$ v, C IV, and Si IV and they sample gas at temperatures near $10^{5} \mathrm{~K}$, which originates where gas near $10^{6} \mathrm{~K}$ cools or interacts with $10^{4} \mathrm{~K}$ gas. For each sight line, we determined over which range of velocities the absorption is likely to be associated with the lower Milky Way corona. We then measured the centroid velocity, line width, and column density for each ion. In many sight lines multiple components can be distinguished, which were separately measured. From these measurements we find that 
1. The centroid velocities of $\mathrm{OVI}, \mathrm{N} v, \mathrm{CIV}$, and $\mathrm{Si} I V$ are very well aligned, with a dispersion in the differences of $<8 \mathrm{~km} \mathrm{~s}^{-1}$.

2. The highly ionized absorption extends to velocities between 30 and $80 \mathrm{~km} \mathrm{~s}^{-1}$ more negative or more positive than the range that can easily be explained by differential galactic rotation. For sight lines in the region near the Galactic north pole, there is an excess of absorption at velocities more negative than $-60 \mathrm{~km} \mathrm{~s}^{-1}$. This excess does not correlate with the IVCs seen in $\mathrm{H}_{\mathrm{I}} 21 \mathrm{~cm}$ emission.

3. We determined line widths by calculating the second moment of the apparent column density profiles, converting to the FWHM. The resulting widths of the $\mathrm{O}$ VI and $\mathrm{N} \mathrm{V}$ lines are on average $60 \mathrm{~km} \mathrm{~s}^{-1}$ and correlate very well (correlation coefficient $\rho=0.97$ ). The average widths of $\mathrm{C}$ IV and Si IV are $42 \mathrm{~km} \mathrm{~s}^{-1}$ (and $\rho=0.94$ ). The other correlations (e.g., between the widths of $\mathrm{O}$ VI and C IV) are slightly less strong $(\rho \sim 0.92)$. The difference between the widths of $\mathrm{O}$ VI/N V and C IV/Si IV is shown to be significant. Since the thermal width of these lines is only $\sim 15 \mathrm{~km} \mathrm{~s}^{-1}$ for gas at a temperature of $10^{5} \mathrm{~K}$, most of the width must be non-thermal.

4. We find that differential galactic rotation leaves its imprint on the velocities of the absorption lines. We predict the locations of the centroids from a simple model in which the gas corotates as one goes up above the disk, and in which the density falls off exponentially with a scale height of $3 \mathrm{kpc}$. Comparing these predictions to the observed centroids we can see the effect of galactic rotation, and we find that the average difference between the data and the prediction from the simple model is $5 \pm 16 \mathrm{~km} \mathrm{~s}^{-1}$.

5. The distributions of the integrated column densities of the highly ionized atoms is relatively narrow, with average and dispersion $14.31 \pm 0.20(\mathrm{O} \mathrm{VI}), 13.48 \pm 0.23(\mathrm{~N} \mathrm{~V})$, $14.17 \pm 0.21$ (C IV), and $13.57 \pm 0.18$ (Si IV). For individual components these values are $14.06 \pm 0.27$ (O VI), $13.30 \pm$ $0.30(\mathrm{~N} \mathrm{v}), 13.83 \pm 0.34(\mathrm{C}$ IV), and $13.24 \pm 0.35$ (Si IV). Note that in the case of $\mathrm{N} \mathrm{v}$ upper limits were omitted for this calculation.

6. Over much of the sky the column densities generally increase with decreasing galactic latitude, but with a dispersion of about 0.17 dex (a factor of 1.5) around the average. The equivalent polar column densities are 14.15, 13.19, 13.89, and 13.32 for O VI, N v, C IV, and Si IV, respectively.

7. Assuming solar abundances and an ionization fraction of about $10 \%$ the polar column densities imply a total hydrogen column density associated with the transition temperature gas of about $10^{18.4} \mathrm{~cm}^{-2}$.

8. In sight lines near the Galactic center and toward the Galactic north pole, the column densities of all ions are enhanced by about 0.3 dex. This suggests enhanced activity at small Galactocentric radii and near the Galactic north pole. We find marginal evidence that this excess is associated with enhanced absorption at negative velocities.

9. At intermediate latitudes in the southern sky, O vi is depressed by about 0.2 dex, but none of the other ions are, indicating a subtle difference in the physical conditions.

10. Determining the ratios of the column densities of the different ions, we find that the distributions of $\mathrm{Nv} / \mathrm{O} \mathrm{VI}$ and Si IV/C IV are rather narrow, with a dispersion of just 0.14 dex $( \pm 40 \%)$, and a spread between the smallest and largest value of $0.6 \mathrm{dex}$ (factor of four). The distributions of other ratios (such as $\mathrm{C}$ IV/O VI) give a larger spread of
0.23 dex $( \pm 70 \%)$. Important average ratios and spreads are (using a linear scale) $\mathrm{N} \mathrm{v} / \mathrm{O} \mathrm{vI}=0.14 \pm 0.04, \mathrm{O} v \mathrm{vI} / \mathrm{N} \mathrm{v}=$ $7.80 \pm 2.90, \mathrm{C}$ IV $/ \mathrm{O}$ VI $=0.71 \pm 0.36, \mathrm{O}$ VI $/ \mathrm{C}$ IV $=1.83 \pm$ $1.15, \mathrm{Si}$ IV $/ \mathrm{C}$ IV $=0.26 \pm 0.08, \mathrm{C}$ IV $/ \mathrm{Si}$ IV $=4.19 \pm 1.47$.

11. We discuss a number of ionization mechanisms, going back to the original papers to extract the detailed predictions. The models we discuss include CIE (from Sutherland \& Dopita 1993; Gnat \& Sternberg 2007), static NIE radiative cooling (Gnat \& Sternberg 2007), radiatively cooling gas flows (Shapiro \& Benjamin 1991, but fully described for the first time in the Appendix), shock ionization (Dopita \& Sutherland 1996), conductive interfaces (Borkowski et al. 1990; Gnat et al. 2010), thick disk supernovae (Shelton 1998), and turbulent mixing layers (Slavin et al. 1993; Kwak \& Shelton 2010). We compare the predictions of ionic ratios for each model with the data in four different ratio-ratio plots, which include $\mathrm{Nv} / \mathrm{O}$ vI, C IV/O vI, or $\mathrm{C}$ IV/N v versus $\mathrm{Si}$ IV/C IV and N v/O vi versus C IV/O vi. These were chosen so that for future observations at least one diagram can be used if data are available for only three of the ions. The historically most used $\mathrm{Nv} / \mathrm{O}$ vi versus $\mathrm{C}$ IV/O vi diagram is the least discriminative of the four.

12. We find that single-phase CIE models fail to explain the observations.

13. The model for static NIE radiative cooling shows that if the gas cools below $T \sim 10^{4.9} \mathrm{~K}$, the predicted ionic ratios are close to the observed values, with $\mathrm{Si}$ IV/C IV underpredicted by about 0.3 dex and $\mathrm{N}$ v/O vi overpredicted by the same amount.

14. In radiatively cooling gas flows, there is a range of flow velocities at which the amount of cooling gas is similar to the observed amount. The best match for all four ions simultaneously requires a flow velocity on the order of $25 \mathrm{~km} \mathrm{~s}^{-1}$. This model predicts that the different ions originate in different parts of the flow, with the pairs $\mathrm{N} \mathrm{v} / \mathrm{O}$ vi and $\mathrm{C}$ IV/Si IV living more closely together with each other than they do with the other ions. The model correctly predicts the range of ratios for $\mathrm{C} I V / \mathrm{NV}$ and $\mathrm{Si}$ IV/C IV, although it overpredicts O vi/N v.

15. Shock ionization models predict far too much $\mathrm{O}$ vi relative to the amount of $\mathrm{N} \mathrm{V}$ and $\mathrm{CIV}$, as well as too much $\mathrm{Nv}$ relative to $\mathrm{C}$ IV, i.e., the predicted ionization state of the gas is too high.

16. Conductive interface models were favored by Sembach et al. (2003) and Fox et al. $(2004,2005)$ to explain the transition temperature ions seen in HVCs. However, Fox et al. $(2004,2005)$ used the full ranges for $\mathrm{N} \mathrm{v} / \mathrm{O} \mathrm{vI}$ and Civ/O vi predicted by Borkowski et al. (1990) for interfaces older than $10^{5} \mathrm{yr}$. Here, we instead follow the evolution of these ratios with time and find that the delineated region is really a strip that lies below the observations. Furthermore, it takes only 1-2 Myr for the ratios to stabilize at a fixed value, compared to an interface lifetime that is $>10 \mathrm{Myr}$. The other three diagrams show that the model strongly underpredicts $\mathrm{Si}$ IV/C IV, while the stabilized values for $\mathrm{Nv} / \mathrm{O}$ vi and $\mathrm{C}$ IV/O vi also tend to be too low. Furthermore, the model predicts total column densities in a single layer of only $\sim 10^{12.5}$ for $\mathrm{O}$ VI, so that $>50$ interfaces are required in each sight line. In light of these considerations, we conclude that the absorption from highly ionized atoms in the Galactic corona probably does not originate in conductive interfaces. 
17. The effects of thick disk supernovae on the ionization state of the tenuous Galactic coronal gas was explored by Shelton (1998). In this model, the $\mathrm{N}$ v/O vi and C IV/O vi ratios evolve slowly at first, but then increase almost linearly over $16 \mathrm{Myr}$ to cover the observed range. Si IV/C IV on the other hand is basically constant over the lifetime of the remnant, but underpredicted by about 0.5 dex. The integrated ionic column densities for O VI, N v, and C IV are only a factor of three to five below the observed values. If a way could be found to increase $N(\mathrm{Si}$ IV) (e.g., including photoionization), this model might fit the observations. However, the number of thick disk supernovae needed to produce the integrated column densities is a factor of a few larger than the number of supernovae observed.

18. The original predictions for turbulent mixing layers by Slavin et al. (1993) cover a range in Si IV/C IV that is much larger than what is observed. The predictions for the other ionic ratios also do not match the data well. On the other hand, a more recent, numerical, model of turbulent mixing layers by Kwak \& Shelton (2010) almost gets most of the ionic ratios correct, although it appears to overpredict the amount of the ions with higher ionization potential (O VI and $\mathrm{N} \mathrm{v}$ ).

19. From the fact that the number of absorption components that can be identified in each sight line is between one and three, combined with the comparisons between the data and predictions from different models, and taking into account the results of our analysis of velocities and column densities, we conclude that the transition temperature gas in the Milky Way corona originates in several (one to five) structures in each sight line. These are more common closer to the plane and have a scale height of $\sim 3 \mathrm{kpc}$. Each structure has a randomly oriented velocity, with a dispersion between different structures of about $75 \mathrm{~km} \mathrm{~s}^{-1}$. In these structures radiative cooling takes place, fast enough for the ionization to be frozen in. Whether the structures originate in a cooling flow or as the result of the mixing of cool and hot gas remains unclear.

20. Comparing the observed ionic ratios in thick disk gas to those seen in disk gas and HVCs, we find that all of these fall in the same regions in the ratio-ratio plots, suggesting a similar origin for the transition temperature gas. In highredshift DLAs, the $\mathrm{O}$ VI, C IV, and Si IV ions also exist in the same proportions, but $\mathrm{N} v$ appears underabundant, which is consistent with the subsolar $\mathrm{N} / \mathrm{O}$ ratios measured in these systems.

Support to B.P.W. for program No. HST-AR-10984.01-A was provided by NASA though a grant from the Space Telescope Science Institute, which is operated by the Association of Universities for Research in Astronomy, Incorporated, under NASA contract NAS5-26555. This paper is also based on observations from the NASA-CNES-CSA Far Ultraviolet Spectroscopic Explorer mission, operated by Johns Hopkins University, supported by NASA contract NAS5-32985. R.A.B. would like to acknowledge the support of NASA Astrophysical Theory grant NNX10AI70G to the University of Wisconsin-Whitewater. We thank Orly Gnat for providing a useful plot of the timescales on which gas cools in her radiative cooling models. We thank Robin Shelton for providing us with the Si IV predictions for the thick disk supernova model. We thank Kyujin Kwak and Robin Shelton for giving us the detailed Myr-by-Myr column density predictions for their turbulent mixing model.

\section{APPENDIX}

\section{A.1. A Cooling Flow Model}

In this appendix, we describe a model to predict the column densities and emissivities of highly ionized atoms. A summary of this model was presented by Shapiro \& Benjamin (1991, 1993) and Benjamin \& Shapiro (1993); the full model is described in Benjamin (1994). This is the model labeled "Cooling Flow" in Figure 11.

The model starts from the realization that a common element of the models used to analyze the column densities of the highly ionized atoms is that they originate in gas that is not thermally stable. As a result, these ions are necessarily associated with flows of heating or cooling material.

Since the heating and cooling timescales of this gas can be shorter than the ionization or recombination timescales, it is necessary to account for non-equilibrium effects when calculating the ionization. Moreover, it is important to consider the effects of ionizing radiation, produced either externally or by the cooling gas itself; this can substantially change the column densities for certain ions under the right circumstances.

Such models of a radiatively cooling flow can be considered a generalization of post-shock flow models. However, unlike the case of radiative shock models, the initial flow velocity and the initial gas temperature are not necessarily coupled. The parameters for a flow model are (1) the initial temperature, (2) the initial density, and (3) the flow's velocity, as well as (4) the density compression evolution. In addition, one must specify an elemental abundance set and a set of atomic data to calculate the NIE and cooling of the gas. In the model calculations described below the abundances used were from Allen (1973); the atomic data were completely described in Benjamin (1994), but come principally from Raymond \& Smith (1977), J. C. Raymond (1987, private communication), Shull \& van Steenberg (1985), and Romanik (1988). There would be a (small) correction associated with adjusting the results to the more recent Asplund et al. (2009) abundances, which changes C IV/O vi by $0.04 \mathrm{dex}$ and Si IV/C IV by $-0.08 \mathrm{dex}$. We include these two changes in all the results shown below. The absolute changes in the abundances are a bit larger $(-0.13$ dex for oxygen, -0.09 dex for carbon, $-0.13 \mathrm{dex}$ for nitrogen, and +0.01 dex for silicon), but (as argued below), a change in the abundance of oxygen will not significantly affect the $\mathrm{O}$ VI column density. For the other elements it is only the change relative to the change in oxygen abundance that matters.

\section{A.2. Cooling Curve}

The effects of the NIE and cooling are encapsulated in the cooling curve. These are given in Figure 12 for equilibrium, isobaric, and isochoric cooling gas, along with the dominant coolants at different temperature ranges. The instantaneous cooling time for a solar metallicity gas in ionization equilibrium with initial temperature $T_{6}=T / 10^{6} \mathrm{~K}$ is $t_{c}=3 \times$ $10^{7} n_{-2}^{-1} T_{6} \mathcal{L}_{-23}{ }^{-1} \mathrm{yr}$, where $n_{-2}=n_{\mathrm{H}} /\left(10^{-2} \mathrm{~cm}^{-3}\right)$ and the normalized cooling rate is $\mathcal{L}_{-23}=\Lambda /\left(n_{\mathrm{e}} n_{\mathrm{H}}\right) /\left(10^{-23} \mathrm{erg} \mathrm{cm}^{-3} \mathrm{~s}^{-1}\right)$. This complicated function can be approximated by functional forms that are good to within $50 \%$ (for the equilibrium case) or even $30 \%$ (for the isobaric and isochoric cases) by the following 


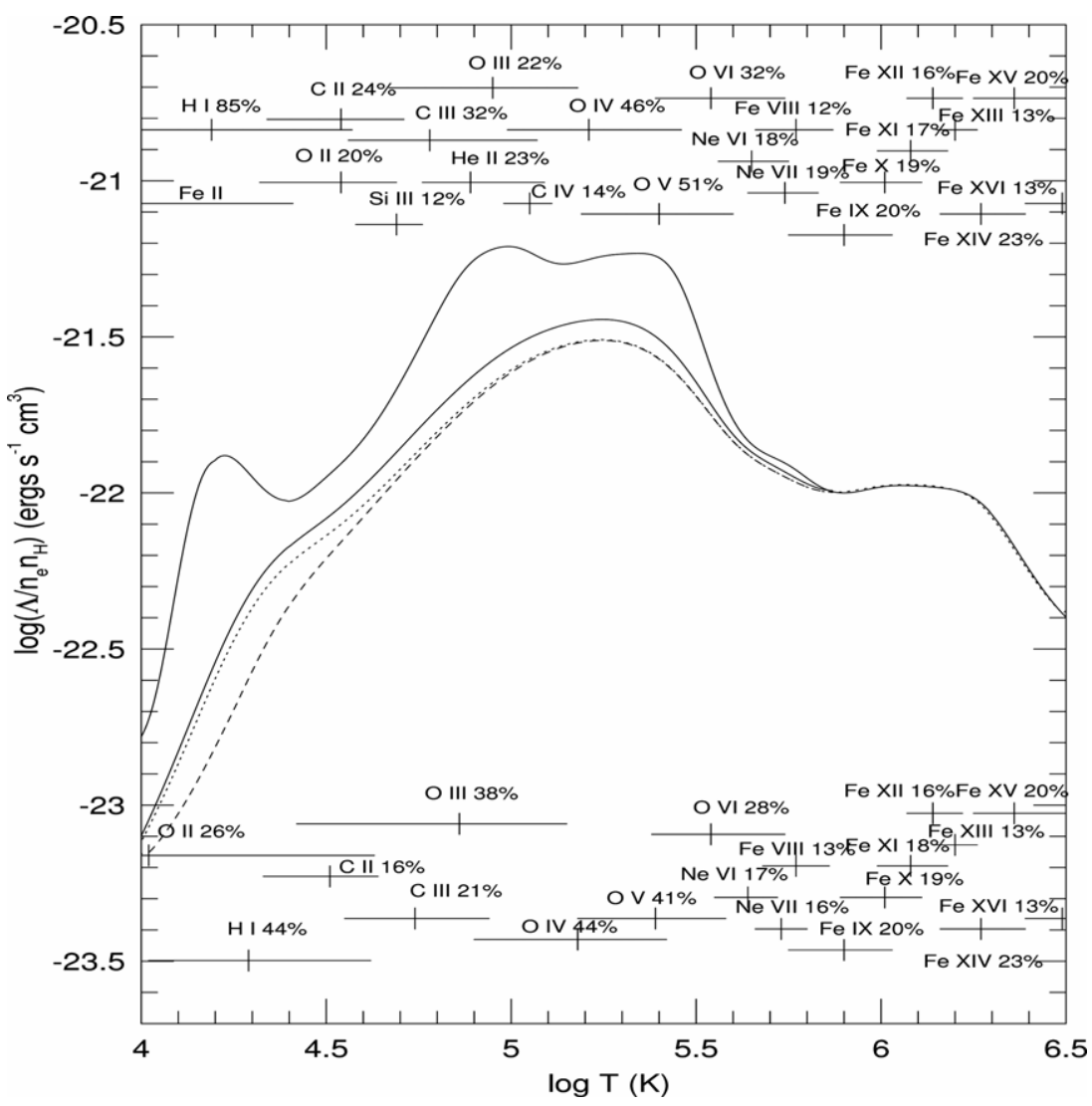

Figure 12. Radiative cooling rate of a solar metallicity gas $\left(\log \left[\Lambda / n_{e} n_{\mathrm{H}}\right]\left(\mathrm{erg} \mathrm{cm}^{-3} \mathrm{~s}^{-1}\right)\right)$ vs. $\log T(\mathrm{~K})$. Three cases are shown: cooling in collisional equilibrium ionization (upper solid curve), isobarically with NIE evolution (lower solid curve), and isochorically with NIE evolution (dotted curve), in the absence of the effects of ionizing radiation. The dashed line shows the change in the isochoric cooling curve when the effects of radiation are accounted for assuming an initial flow velocity of $v_{0}=30 \mathrm{~km} \mathrm{~s}^{-1}$. These curves are very similar in shape and level to the ones used by others (see, e.g., Sutherland \& Dopita 1993; Gnat \& Sternberg 2007). The horizontal bars at the top of the graph indicate the temperature regime in which the labeled ion contributes more than $10 \%$ of the total cooling rate for gas cooling in ionization equilibrium. The temperature at which this ion makes its maximum fractional contribution to the cooling rate is denoted by a vertical tick mark and the percentage is noted. The lower bars show the same quantities but for non-equilibrium, isobarically cooling gas.

formulae:

$$
\mathcal{L}_{-23}(T)=\left\{\begin{array}{c}
13.4 T_{6}^{-0.74} \\
10^{5} \mathrm{~K} \lesssim T \lesssim 2 \times 10^{7} \mathrm{~K} \text { (equilibrium) } \\
36.3 \exp \left[-3.4\left(\log _{10}(T)-5.23\right)^{2}\right] \\
10^{4.4} \mathrm{~K} \lesssim T \lesssim 10^{6} \mathrm{~K} \text { (isobaric) } \\
31.2 \exp \left[-3.4\left(\log _{10}(T)-5.23\right)^{2}\right] \\
10^{4.4} \mathrm{~K} \lesssim T \lesssim 10^{6} \mathrm{~K} \text { (isochoric) }
\end{array}\right\} .
$$

When the gas cools to a temperature $T \sim 10^{4.4} \mathrm{~K}$, the dominant source of cooling is collisional excitation of $\mathrm{H} \operatorname{Ly} \alpha$, which is extremely sensitive to the ionization fraction of hydrogen, and thus depends sensitively upon the assumed value of the incident ionizing flux.

\section{A.3. Structure of the Flow}

The initial conditions of the one-dimensional model are marked by a flow boundary. On one side of this boundary the gas has some high initial temperature $T_{0}\left(>10^{6} \mathrm{~K}\right)$, and on the other side the gas has started to cool due to radiative losses. Hot material flows through the boundary, and moves away from it. This sets up a steady-state temperature and ionization-level gradient along the direction of the flow. The rate of mass $(M)$ flowing through per unit area $(A)$ equals $\dot{M} / A=\bar{m} n_{\mathrm{H}, 0} v_{0}$, where $\bar{m}$ is the mean mass per hydrogen atom (which depends on the metallicity), $n_{\mathrm{H}, 0}$ is the initial hydrogen particle density, and $v_{0}$ is the flow velocity. This velocity is defined in the (unique) reference frame where the particle flux remains constant.

As the gas cools, the loss of thermal energy reduces its pressure, and thus its density should increase. However, the actual pressure profile along the flow is also affected by the constant addition of new material, by geometrical constraints (e.g., expansion) and/or by magnetic fields. For simplicity, we only consider two bounding cases for the density evolution of the gas as it travels along the flow: isobaric (constant pressure) and isochoric (constant volume and thus constant density). In the isobaric case the temperature drops along the flow, but it is assumed that the density increases proportionally, such that the pressure is constant. This occurs, for example, in a nonmagnetized steady-state environment, where the sound crossing time is shorter than cooling time. Most of the other models that are being considered elsewhere in this paper (turbulent mixing, conductive interfaces, radiative shocks) are also characterized by pressure equilibrium. In the isochoric case, the temperature drops, but the gas does not compress. This occurs, for example, if magnetic pressure exceeds the thermal pressure in the gas, or if the flow is unconstrained by external pressure and allowed to diverge. For either of these cases (isobaric or isochoric), fixing the pressure or density profile along the flow fixes the temperature profile as function of the time elapsed since the material passed the boundary. 
Table 3

Cooling Flow Parameters (without Photoionization Effects)

\begin{tabular}{|c|c|c|c|c|c|c|c|c|c|}
\hline \multirow[t]{2}{*}{ Case } & \multirow[t]{2}{*}{$X^{\mathrm{a}}$} & \multirow[t]{2}{*}{$\phi_{\mathrm{HI}_{\mathrm{I}}}^{\mathrm{b}}$} & \multicolumn{5}{|c|}{$\mu_{i, 6}{ }^{\mathrm{c}}$} & \multicolumn{2}{|c|}{$\iota_{j, 3} \mathrm{~d}$} \\
\hline & & & Si IV & CIV & $\mathrm{N} \mathrm{v}$ & O VI & Ne VIII & C IV $1548 \AA$ & O vi $1031 \AA$ \\
\hline Isobaric & 112 & 7.0 & 0.037 & 1.35 & 1.67 & 41.40 & 27.93 & 4.0 & 25.6 \\
\hline Isochoric & 1 & 4.3 & 0.59 & 8.39 & 4.26 & 62.33 & 23.87 & 2.5 & 15.9 \\
\hline
\end{tabular}

Notes.

${ }^{\text {a }}$ Density compression of gas, $n_{\mathrm{H}} / n_{\mathrm{H}, \mathrm{o}}$ between $T_{\mathrm{o}}=10^{6} \mathrm{~K}$ and $T \sim 10^{4} \mathrm{~K}$.

${ }^{\mathrm{b}}$ Hydrogen-ionizing photons produced per $\mathrm{H}$ atom in flow.

${ }^{\mathrm{c}}$ Column density normalized to initial flow velocity, $\mu_{i}=N_{i} / v_{0}$, in units of $10^{6} \mathrm{~cm}^{-3} \mathrm{~s}$ when photoionization is neglected.

${ }^{\mathrm{d}}$ Emission line intensity of resonance doublet normalized to flow mass flux, $\iota_{j,-3}=I_{j} /\left(n_{\mathrm{H}, \mathrm{o}} v_{0}\right)$ in units of $10^{3} \mathrm{photons} \mathrm{sr}^{-1}$.

In order to compare the model predictions to observations, we derive the column densities of the different ions along sight lines along the flow direction, i.e., perpendicular to the boundary. The column density of any given ion is equal to the integral of the ion's density times the length of the segment in the flow where the ion is present. Given a flow velocity $v_{0}$, and an amount of time $d t$ spent at some given temperature, the segment's length is $v_{0} d t$. Each ion $i$ has an elemental abundance, $A_{i}$. Its ionization fraction after some amount of time, $t$, since passing through the boundary is $f_{i}(t)$, as derived from the radiative cooling calculation. Thus, the ion's volume density at time $t$ since crossing the boundary is $f_{i}(t) A_{i} n_{\mathrm{H}, 0}$. This implies a column density along the flow direction equal to

$$
N_{i}=\int_{0}^{t} f_{i}\left(t^{\prime}\right) A_{i} v_{0} d\left(n_{\mathrm{H}, 0} t^{\prime}\right)=v_{0} \mu_{i},
$$

where this equation defines $\mu_{i}$ as a constant of the flow for each ion. Values for $\mu_{i}$ for a number of ions can be found in Table 3.

The fact that the column densities are proportional to the flow velocity, but do not depend on the density or ionization structure can be understood as follows. Start with a zone in the flow at a given temperature. If the density is high, the cooling time is short, and thus the thickness of the zone is small. At lower densities, the cooling time is long and thus the zone is thicker. The thickness of zone is thus proportional to the product of the density, the flow velocity, and the time spent at a given temperature. Since the flow velocity is assumed to be a constant of the flow, the time evolution of a fluid element only depends on the product $\left(n_{\mathrm{H}, 0} t\right)$. This is referred to as the fluence, and it may be interpreted indirectly as a scaled spatial coordinate. The resulting scaling property allows us to run a case for a fixed density, and then rescale our results for an arbitrary density. We note that other models (radiative shocks, mixing layers, conductive interfaces) also exhibit scaling relations.

The same argument shows that the column density predictions will not change much with metallicity. Lowering the metal abundance increases the cooling time by the same factor, since the principal coolants are ions of oxygen. This means that the predictions for oxygen ions are nearly independent of oxygen abundances. To first order, the column densities for the other ions will also stay constant. However, a proper handling of this issue would require calculations using a number of different assumed abundances.

\section{A.4. Photoionization}

A crucial (and unique) aspect of the model described here is that it takes into account the ionizing radiation that is produced by the hot, upstream gas. Here, we discuss a few general implications of including photoionization.
For a given cooling history, the quantity $\phi_{v}=F_{v} /\left(n_{\mathrm{H}, 0} v_{0}\right)$ is a constant of the flow, where $F_{v}$ is the photon flux emitted by the cooling gas at a given frequency and $\left(n_{\mathrm{H}, 0} v_{0}\right)$ is the particle flux. The effectiveness of photoionization depends on the photoionization parameter, $U_{i}=n_{\gamma, i} / n_{\mathrm{H}}$, where $n_{\gamma, i}=$ $\left(F_{i} / c\right)$ is the density of photons capable of ionizing some ion $i$. Therefore, we can express the ionization parameter at a given point in the cooling flow as $U_{i}=F_{i} /\left(c n_{\mathrm{H}}\right)=\left(\Phi_{i} / X\right)\left(v_{0} / c\right)$, where $X=n_{\mathrm{H}} / n_{\mathrm{H}, 0}$ is the factor by which the flow has compressed, and $\Phi_{i}$ is the integral of $\phi_{v}$ for photons that can ionize ion $i$.

For example, for a flow velocity of $v_{0}=60 \mathrm{~km} \mathrm{~s}^{-1}$, the ionization parameter in the photoionized part of an isochoric flow, where the hydrogen ionizing photons per $\mathrm{H}$ atom is $\Phi_{\mathrm{H}_{\mathrm{I}}}=4.3$ and the gas compression is $X=1$, will be $\log U_{\mathrm{H}_{\mathrm{I}}}=$ -3.1 . In contrast, for isobarically cooling gas, where $\Phi_{\mathrm{H}_{\mathrm{I}}}=$ 7.0 and $X=112$, the corresponding ionization parameter is $\log U_{\mathrm{HI}}=-4.9$. This low-ionization parameter in isobarically cooling gas implies that there is no photoionization boost in this case. Despite the increased number of ionizing photons produced per atom in the cooling gas (due to $p d V$ work), the compression lowers the effectiveness of photoionization.

The width of the photoionization zone, where the radiation from the hot section is reprocessed, can be estimated by equating the number flux of ionizing photons produced by the hot flow to the number of recombinations per hydrogen atom per second in the photoionized zone. Using the scaling relationships above this yields $L \sim v_{0} \Phi_{\mathrm{HI}} /\left(n_{\mathrm{H}, 0} \alpha_{\mathrm{H}} X^{2}\right)$, where $\alpha_{\mathrm{H}}$ is the Case A radiative recombination coefficient of hydrogen. Our numerically calculated lengths agree well with this analytical estimate, yielding $L \sim 4 / n_{-2}$ pc for the isobaric case and $L \sim$ $800 / n_{-2} \mathrm{pc}$ for the isochoric case with $v_{0}=30 \mathrm{~km} \mathrm{~s}^{-1}$. The corresponding flow times are $t=3.3 \times 10^{5} / n_{-2}$ yr for the isobaric case and $2.5 \times 10^{7} / n_{-2}$ yr for the isochoric case.

The inclusion of photoionization produces a steeper than linear relationship between the column density and $v_{0}$, as demonstrated in Figure 15. In this case, the column density can be written as $N_{i}=v_{0} \mu_{i} b_{i}\left(v_{0}\right)$, where $b_{i}\left(v_{0}\right)$ is a photoionization boost for ion $i$, which increases with flow velocity.

\section{A.5. Column Density Predictions}

The cumulative column density as a function of (normalized) position is shown in Figure 13 for an isochoric flow with $v_{0}=$ $30 \mathrm{~km} \mathrm{~s}^{-1}$, both with and without photoionization. Figure 14 shows the temperature evolution and the ionization fractions as function of position. In this example, the spatial thickness of the zone containing the highly ionized gas is $\sim 100 n_{-2}$ pc. When the effects of photoionization are added in, the path length 


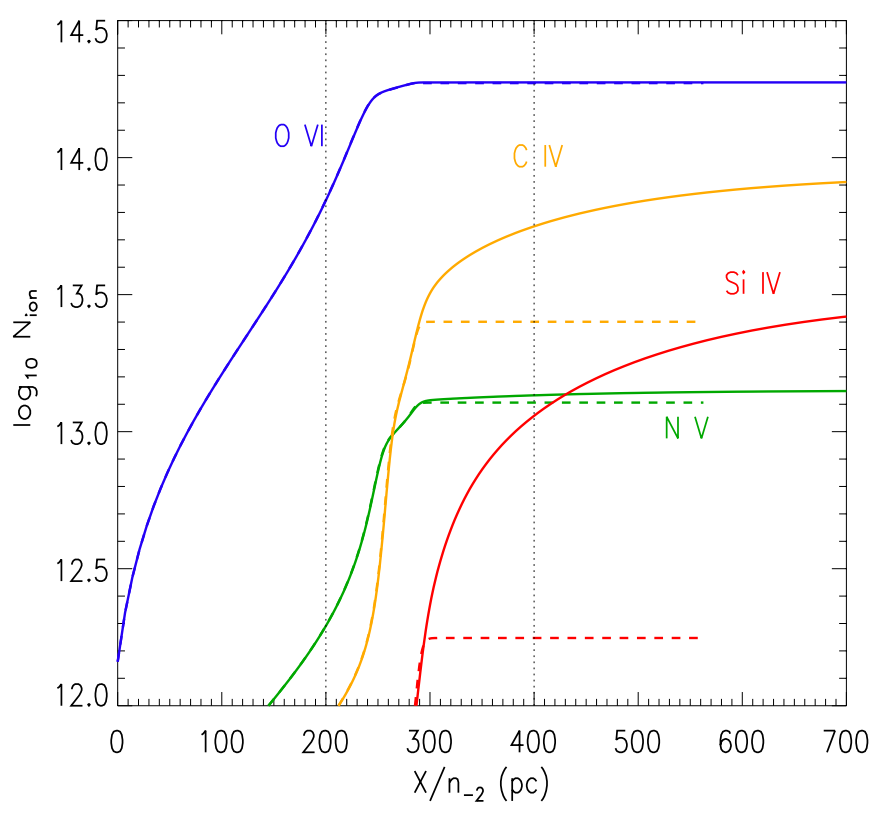

Figure 13. Cumulative column density of highly ionized gas as a function of (normalized) path length through an isochoric radiative cooling flow with $v_{0}=$ $30 \mathrm{~km} \mathrm{~s}^{-1}$. The solid and dotted lines show the column density with and without the inclusion of photoionization from the radiation field produced by the cooling gas.

occupied by C IV and particularly Si IV is extended by a factor of two to eight.

Figure 15 shows the predicted column densities as function of the flow velocity, $v_{0}$. There are three useful insights that one can take from this figure. First, when comparing models to observations, column density ratios are not necessarily independent of total column densities. Models which successfully produce the observed ratios of column densities might do so in increments so large as to be inconsistent with the estimated column per component or so small as to require hundreds of interfaces along the line sight (as is, e.g., the case for conductive interface layers). Second, for any reasonable set of parameters, the $\mathrm{O}$ VI and $\mathrm{N} v$ ions originate only in the thermal zone and are basically independent of the radiation field. This is because their ionization potentials are much higher than the typical photon energy (I.P., for going from $\mathrm{O} v$ to $\mathrm{O}$ vi $113.9 \mathrm{eV}$, for going from $\mathrm{N}$ IV to $\mathrm{Nv} 77.47 \mathrm{eV})$. Third, if there is resistance to density compression (or a higher radiation field than that produced in the cooling gas alone), photoionization can become important in contributing to the column densities of Si IV (I.P. $33.49 \mathrm{eV}$ ) and C IV (I.P. $47.89 \mathrm{eV}$ ).

Figure 15 shows that the observed ranges of column densities through the Galactic corona for $\mathrm{C}$ IV and $\mathrm{Si}$ IV both are consistent with flow velocities between 15 and $35 \mathrm{~km} \mathrm{~s}^{-1}$. The range for $\mathrm{N} \mathrm{v}$ (15-65 $\mathrm{km} \mathrm{s}^{-1}$ ) overlaps with this, but in some directions higher flow velocities would be needed to produce the observed $\mathrm{N} v$. On the other hand, such flows would predict too much $\mathrm{O}$ VI. Nevertheless, since this cooling flow is modeled as a simplified one-dimensional flow, it is encouraging that there is a range of flow velocities for which all four column densities are similar to the observed values.

It is relatively easy to understand why the thermal and ionization structure of the cooling gas is independent of density or metallicity. However, if photoionization is important, one might expect the volume density to influence the column densities. However, the photon flux from the flow is linearly proportional to the particle flux. This means that cooling flows

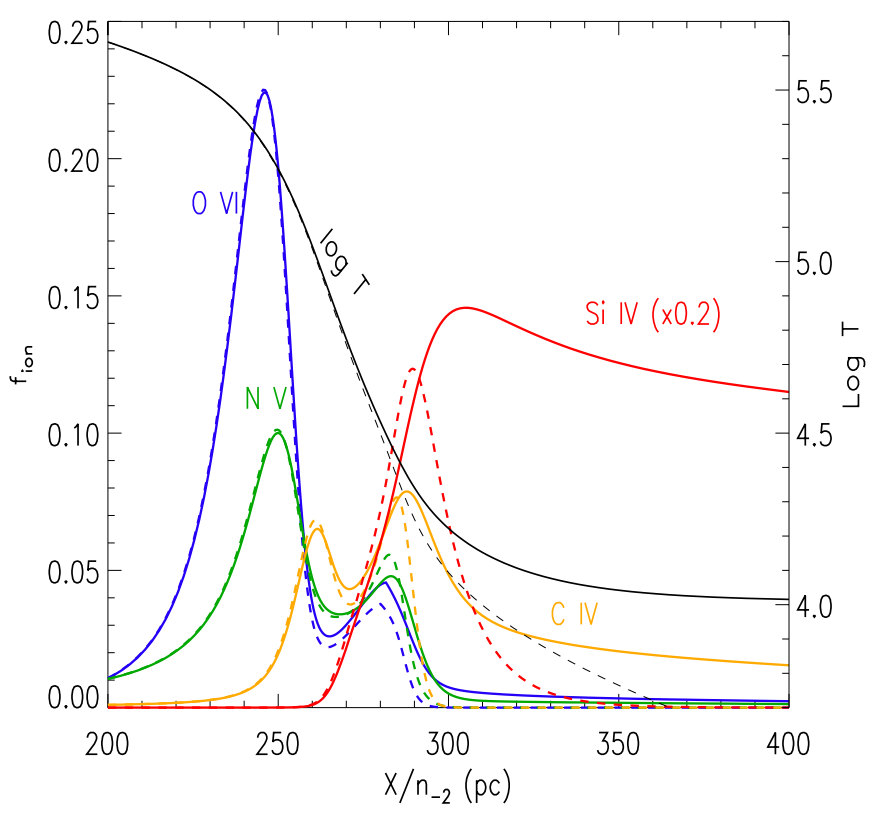

Figure 14. Ionization fractions (left axis) and temperature (right axis) as a function of normalized position for isochorically cooling gas with $v_{0}=$ $30 \mathrm{~km} \mathrm{~s}^{-1}$ for cases that include (solid) and do not include (dashed) the effects of photoionization. For clarity, the ionization fraction of Si IV has been multiplied by 0.2 . The thickness of the zones scales linearly with $v_{0}$, although the zones where C IV and Si IV occur become more extended due to the enhanced photoionization.

produce a fixed number of ionizing photons per hydrogen atom, independent of the density. Thus, the column density predictions do not depend on the absolute value of the volume density (although they do depend on the density structure).

\section{A.6. Emissivity Predictions}

In general, the density of the flow can be determined by comparing a column density $\left(\propto n_{\mathrm{H}}\right)$ with an emission line (or broadband) intensity $\left(\propto n_{\mathrm{H}}^{2}\right)$. With a model, any absorption/emission pair can be chosen. In the original Galactic fountain model of Shapiro \& Field (1976), for example, the combination of matching a model of soft X-ray emission and $\mathrm{O}$ VI column densities yielded a density large enough that it indicated that outflowing gas would cool and fall back before reaching an equilibrium scale height, suggesting that hot gas in the disk might establish a convective-radiative cycle. The least model-dependent results come from comparing the emission and absorption from a single ion. Martin \& Bowyer (1990), for example, used the absorption/emission from C IV. However, the chief uncertainty in this approach is the possibility that a significant fraction of $\mathrm{C}$ IV could be produced in a photoionized region and therefore absorbing but not emitting. The body of $\mathrm{O}$ VI absorption and emission measurements now available from FUSE alleviates this problem, since it is expected that $\mathrm{O}$ VI absorption and emission arise in the same spatial region.

Since the intensity of any emission line is proportional to the particle flux, $I_{j}=\left(n_{\mathrm{H}, 0} v_{0}\right) b\left(v_{0}\right) \iota_{j}$, and the column densities are proportional to the flow velocity only, $N_{i}=v_{0} a_{i}\left(v_{0}\right) \mu_{i}$, one can use the comparison of the two quantities to solve for the initial density of the flow, $n_{\mathrm{H}, 0}=\left(I_{j, \mathrm{obs}} / N_{i, \mathrm{obs}}\right)\left(\mu_{i} / \iota_{j}\right)\left(a\left(v_{0}\right) / b\left(v_{0}\right)\right)$, where $I_{j \text {,obs }}$ and $N_{i, \text { obs }}$ are the observed emission line intensity and column density from a region of interest. The constant of proportionality between the particle flux and emission line intensity, $\iota_{j}$, is given in Table 3 for selected lines. The photoionization boost term for the intensities is $b\left(v_{0}\right)$. For infrared 


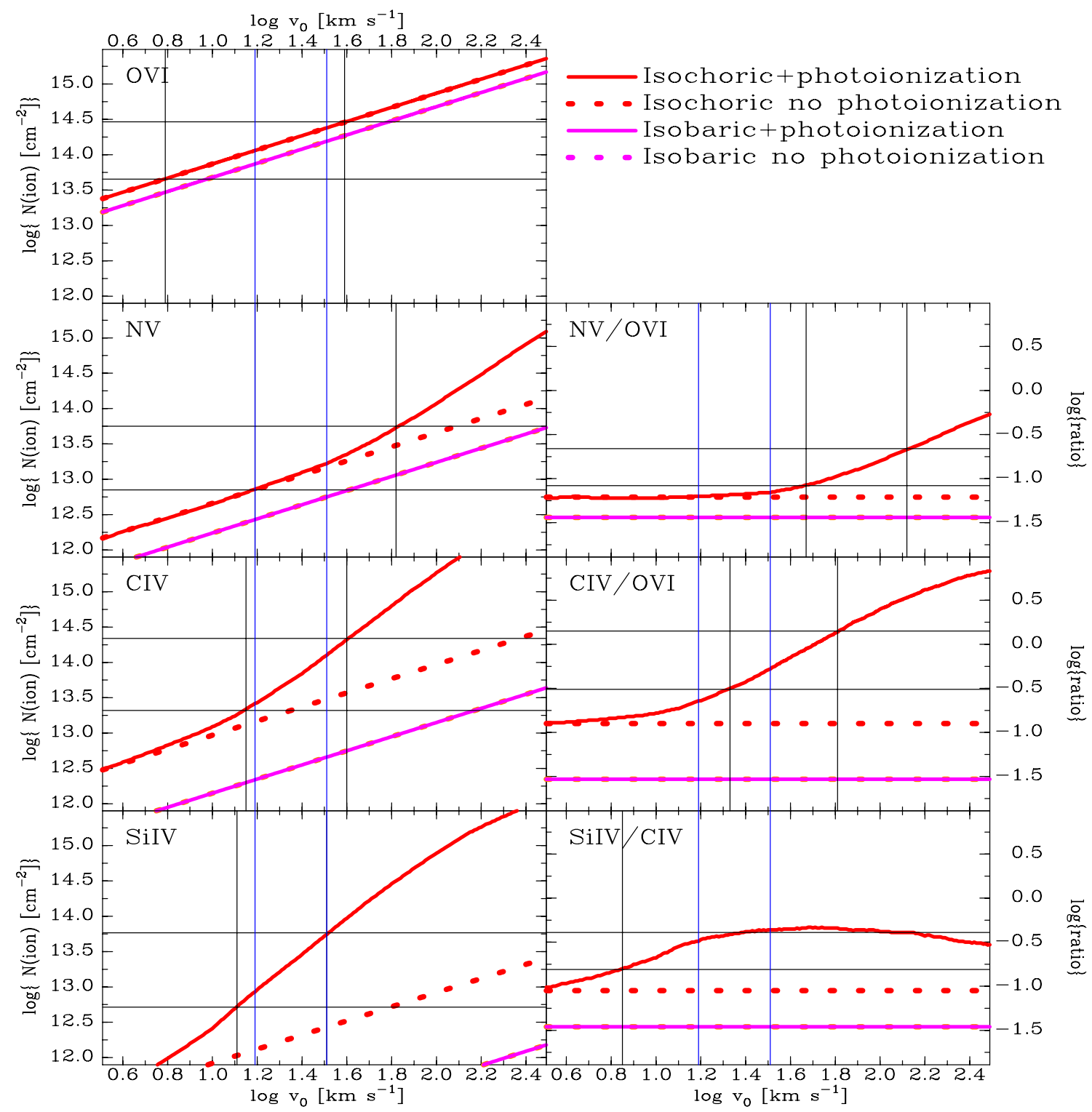

Figure 15. Left panels: column densities as a function of the logarithm of the flow velocity, $v_{0}$, for cases with isobaric cooling (magenta line) and for isochoric cooling with and without photoionization (red solid and red dashed line). Note that the column densities are linearly proportional to flow velocity for low velocities. Note the significant photoionization boost for $\mathrm{N} v, \mathrm{C}$ IV, and Si IV, setting in at $\log v_{0} \sim 1.4,1.0$, and 0.7 , respectively. Right panels: selected column density ratios as a function of the logarithm of the flow velocity. In all panels the observed $1.5 \sigma$ range around the average ionic column density or ratio observed in the Galactic corona is shown with horizontal lines. The averages and dispersions can be found in Sections 4.5 and 4.8. In all panels, vertical lines show the range of velocities, $v_{0}$, for which the predicted column density or ratio falls within the observed range. The solid blue vertical lines show the velocity range within which all four column density predictions fit the observed range $\left(15-32 \mathrm{~km} \mathrm{~s}^{-1}\right)$.

and optical lines, this correction can be quite significant. However, for ultraviolet and X-ray resonance lines, this correction is negligible, as the temperature in the photoionized zone is too low to excite these higher energy transitions. For example, the C IV $\lambda \lambda 1548,1550$ doublet is enhanced by less than $10 \%$ in the photoionized isochoric flows, for example.

Combining the results for the emission and absorption, and scaling these numbers to reasonable values, $N_{i, 14}=$ $N_{i} / 10^{14} \mathrm{~cm}^{-2}$ and $I_{j, 1000}=I_{j} /\left(1000\right.$ photons s $\left.\mathrm{s}^{-1} \mathrm{~cm}^{-2} \mathrm{sr}^{-1}\right)$ (line units $=\mathrm{LU}$ ), we can solve for the initial flow density,

$n_{\mathrm{H}, 0}=\left(10^{-3} \mathrm{~cm}^{-2}\right)\left\{\begin{array}{c}(0.34 / 3.31) a_{\mathrm{C} \mathrm{Iv}}\left(v_{0}\right) I_{\mathrm{CIv}, 1000} / N_{\mathrm{CIv}, 14} \\ \quad \text { isobaric/isochoric }) \\ (1.62 / 3.91) a_{\mathrm{O} \mathrm{vI}}\left(v_{0}\right) I_{\mathrm{O} \text { vi } 1000} / N_{\mathrm{O} \mathrm{vI}, 14} \\ \text { (isobaric/isochoric) }\end{array}\right\}$, where the intensity lines are the combined intensities of the resonance doublets of $\mathrm{O}$ VI and CIV. In this above equation, we neglect the (small) photoionization boost, $b_{i}\left(v_{0}\right)$ to the UV emission lines, and $a_{\mathrm{OVI}}\left(v_{0}\right) \sim 1$. Note that since the isochoric case does not compress, it must have a higher initial density to produce the same emergent intensity as an isobaric flow.

Observations of $I(\mathrm{O} \mathrm{VI})$ fall in the range 3000-7000 line units (Dixon \& Sankrit 2008), while the $1 \sigma$ range of $N(\mathrm{O} \mathrm{vI})$ is (1.3-3.0) $\times 10^{14} \mathrm{~cm}^{-2}$ (this work). Unfortunately, there are not enough simultaneous measurements to judge whether these two quantities are correlated. Moreover, the intensity must be corrected for the extinction along the line of sight, and both quantities must be estimated for the same physical path length, cf. Lei et al. (2009) and Shelton et al. (2010). Just using the values above to bracket the possible range of densities, we find that for isochoric flows, $n_{\mathrm{H}, 0}=(0.3-1.5) \times 10^{-2} \mathrm{~cm}^{-3}$. 
Using a mean $\mathrm{O}$ vi temperature of $\log T=5.3$ gives a thermal pressure in the O VI zone of $p / k=600-3000 \mathrm{~cm}^{-3} \mathrm{~K}$. For both isobaric and isochoric cases, the radiative cooling models predict $I(\mathrm{O}$ VI) to be $\sim 6$ times greater than $I(\mathrm{C}$ IV) (in line units). There are very few directions toward which both lines have been estimated, Shelton et al. (2010) combined SPEAR data for C IV with O vI from FUSE, finding a ratio $\sim 1$. Using just SPEAR data, Welsh et al. (2007) find ratios $<1$ to $\sim 3$ in directions toward the north Galactic cap. These ratios indicate a larger $\mathrm{C}$ IV abundance or higher O VI abundances in thermal (nonphotoionized) zones than expected from cooling gas. However, until more and especially more detailed observations are done, it will remain unclear how serious this discrepancy is.

\section{REFERENCES}

Allen, C. W. 1973, Astrophysical Quantities (London: Athlone)

Asplund, M., Grevesse, N., Sauval, A. J., \& Scott, P. 2009, ARA\&A, 47, 481

Begelman, M. C., \& Fabian, A. C. 1990, MNRAS, 244, 26P

Benjamin, R. A. 1994, PhD thesis, Univ. Texas

Benjamin, R. A., \& Shapiro, P. R. 1993, in UV and X-ray Spectroscopy of Laboratory and Astrophysical Plasmas, ed. E. H. Silver \& S. M. Kahn (Cambridge: Cambridge Univ. Press), 280

Borkowski, K. J., Balbus, S. A., \& Fristrom, C. C. 1990, ApJ, 355, 501

Bowen, D. V., Jenkins, E. B., Tripp, T. M., et al. 2008, ApJS, 176, 59

Bregman, J. N. 1980, ApJ, 236, 577

Bunner, A. N., Coleman, P. L., Kraushaar, W. L., McCammon, D., \& Williamson, F. O. 1973, ApJ, 179, 781

Collins, J. A., Shull, J. M., \& Giroux, M. K. 2004, ApJ, 605, 216

Collins, J. A., Shull, J. M., \& Giroux, M. K. 2005, ApJ, 623, 195

Cowie, L. L., Jenkins, E. B., Songaila, A., \& York, D. C. 1979, ApJ, 232, 467

Cowie, L. L., Taylor, W., \& York, D. G. 1981, ApJ, 248, 528

de Avillez, M. A., \& Breitschwerdt, D. 2005, ApJ, 634, L65

Dixon, W. V. D., \& Sankrit, R. 2008, ApJ, 686, 1162

Dopita, M. A., \& Sutherland, R. S. 1996, ApJS, 102, 161

Edgar, R. J., \& Chevalier, R. A. 1986, ApJ, 310, L27

Esquivel, A., Benjamin, R. A., Lazarian, A., Cho, J., \& Leitner, S. N. 2006, ApJ, 648, 1043

Everett, J. E., Zweibel, E. G., Benjamin, R. A., et al. 2008, ApJ, 674, 258

Fox, A. J., Petitjean, P., Ledoux, C., \& Srianand, R. 2007, A\&A, 465, 171

Fox, A. J., Prochaska, J. X., Ledoux, C., et al. 2009, A\&A, 503, 731

Fox, A. J., Savage, B. D., Sembach, K. R., et al. 2003, ApJ, 582, 793

Fox, A. J., Savage, B. D., \& Wakker, B. P. 2006, ApJS, 165, 279

Fox, A. J., Savage, B. D., Wakker, B. P., et al. 2004, ApJ, 602, 738

Fox, A. J., Wakker, B. P., Savage, B. D., et al. 2005, ApJ, 630, 332

Fox, A. J., Wakker, B. P., Smoker, J. V., \& Richter, P. 2010, ApJ, 718, 1046

Fraternali, F., Oosterloo, T. A., \& Sancisi, R. 2004, A\&A, 424, 485

Ganguly, R., Sembach, K. R., Tripp, T. M., \& Savage, B. D. 2005, ApJS, 157, 253

Gnat, O., \& Sternberg, A. 2007, ApJS, 168, 213

Gnat, O., \& Sternberg, A. 2009, ApJ, 693, 1514

Gnat, O., Sternberg, A., \& McKee, C. F. 2010, ApJ, 718, 1315

Heald, G. H., Rand, R. J., Benjamin, R. A., \& Bershady, M. A. 2006, ApJ, 647, 1018

Heald, G. H., Rand, R. J., Benjamin, R. A., \& Bershady, M. A. 2007, ApJ, 663, 933

Henley, D. B., Shelton, R. L., Kwak, K., Joung, M. R., \& Mac Low, M.-M. 2010, ApJ, 723, 935

Houck, J. R., \& Bregman, J. N. 1990, ApJ, 352, 506

Howk, J. C., Savage, B. D., Sembach, K. R., \& Hooper, C. G. 2002, ApJ, 572, 264

Howk, J. C., Sembach, K. R., \& Savage, B. D. 2003, ApJ, 586, 249

Hulsbosch, A. N. M., \& Wakker, B. P. 1988, A\&AS, 75, 191

Indebetouw, R., \& Shull, J. M. 2004a, ApJS, 605, 205

Indebetouw, R., \& Shull, J. M. 2004b, ApJ, 607, 309

Jenkins, E. B. 1978a, ApJ, 219, 845

Jenkins, E. B. 1978b, ApJ, 220, 107

Jenkins, E. B., \& Meloy, D. A. 1974, ApJ, 193, L121

Jenkins, E. B., Oegerle, W. R., Gry, C., et al. 2000, ApJ, 538, L81

Jenkins, E. B., Tripp, T. M., Fitzpatrick, E. L., et al. 1998, ApJ, 492, 147
Kahn, F. D. (ed.) 1981, Investigating the Universe: Papers presented to Zdenek Kopal on the Occasion of His Retirement, September 1981 (Dordrecht: Reidel), 1

Kalberla, P. M. W., Burton, W. B., Hartmann, D., et al. 2005, A\&A, 440, 775

Keeney, B. A., Danforth, C. W., Stocke, J. T., et al. 2006, ApJ, 646, 951

Kim-Quijano, J., et al. 2007, STIS Instrument Handbook, Version 8.0 (Baltimore, MD: STScI)

Knauth, D. C., Howk, J. C., Sembach, K. R., Lauroesch, J. T., \& Meyer, D. M. 2003, ApJ, 592, 946

Kuntz, K. D., \& Danly, L. 1996, ApJ, 457, 703

Kwak, K., \& Shelton, R. S. 2010, ApJ, 719, 523

Lehner, N., \& Howk, J. C. 2010, ApJ, 709, L138

Lehner, N., Jenkins, E. B., Gry, C., et al. 2003, ApJ, 595, 858

Lehner, N., Zech, W. F., Howk, J. C., \& Savage, B. D. 2011, ApJ, 727, 461

Lei, S., Shelton, R. L., \& Henley, D. B. 2009, ApJ, 699, 1891

Martin, C., \& Bowyer, S. 1990, ApJ, 350, 242

Münch, G. 1952, PASP, 64, 312

Münch, G., \& Zirin, H. 1961, ApJ, 133, 11

Oegerle, W. R., Jenkins, E. B., Shelton, R. L., Bowen, D. V., \& Chayer, P. 2005, ApJ, 622, 377

Oegerle, W. R., Tripp, T. M., Sembach, K. R., et al. 2000, ApJ, 538, L23

Oosterloo, T. A., Fraternali, F., \& Sancisi, R. 2007, AJ, 134, 1019

Petitjean, P., Ledoux, C., \& Srianand, R. 2008, A\&A, 480, 349

Raymond, J. C., \& Smith, B. W. 1977, ApJS, 35, 419

Richter, P. 2006, Rev. Mod. Astron., 19, 31

Rogerson, J. B., York, D. G., Drake, J. F., et al. 1973, ApJ, 181, L110

Romanik, C. J. 1988, ApJ, 330, 1022

Savage, B. D., \& de Boer, K. S. 1979, ApJ, 230, L77

Savage, B. D., \& de Boer, K. S. 1981, ApJ, 243, 460

Savage, B. D., Edgar, R., \& Diplas, A. 1990, ApJ, 361, 107

Savage, B. D., \& Lehner, N. 2006, ApJS, 162, 134

Savage, B. D., \& Massa, D. 1987, ApJ, 314, 380

Savage, B. D., Meade, M., \& Sembach, K. R. 2001a, ApJS, 136, 631

Savage, B. D., \& Sembach, K. R. 1991, ApJ, 379, 245

Savage, B. D., \& Sembach, K. R. 1994, ApJ, 434, 145

Savage, B. D., Sembach, K. R., \& Howk, J. C. 2001b, ApJ, 547, 907

Savage, B. D., Sembach, K. R., \& Lu, L. 1995, ApJ, 449, 145

Savage, B. D., Sembach, K. R., \& Lu, L. 1997a, AJ, 113, 2158

Savage, B. D., Sembach, K. R., \& Tripp, T. M. 1997b, ApJ, 480, 216

Savage, B. D., Sembach, K. R., Wakker, B. P., et al. 2003, ApJS, 146, 125

Savage, B. D., \& Wakker, B. P. 2009, ApJ, 702, 1472

Savage, B. D., Wakker, B. P., Fox, A. J., \& Sembach, K. R. 2005, ApJ, 619 863

Sembach, K. R. 1994, ApJ, 434, 244

Sembach, K. R., Howk, J. C., Savage, B. D., Shull, J. M., \& Oegerle, W. R. 2001, ApJ, 561, 573

Sembach, K. R., \& Savage, B. D. 1992, ApJS, 83, 147

Sembach, K. R., \& Savage, B. D. 1994, ApJ, 431, 201

Sembach, K. R., Savage, B. D., \& Cardelli, J. A. 1994a, ApJ, 420, 183

Sembach, K. R., Savage, B. D., \& Hurwitz, M. 1999, ApJ, 524, 98

Sembach, K. R., Savage, B. D., \& Jenkins, E. B. 1994b, ApJ, 421, 585

Sembach, K. R., Savage, B. D., \& Lu, L. 1995, ApJ, 439, 672

Sembach, K. R., Savage, B. D., \& Massa, D. 1990, ApJ, 355, 114

Sembach, K. R., Savage, B. D., \& Massa, D. 1991, ApJ, 372, 81

Sembach, K. R., Wakker, B. P., Savage, B. D., et al. 2003, ApJS, 146 , 165

Shapiro, P. R., \& Benjamin, R. 1991, PASP, 103, 923

Shapiro, P. R., \& Benjamin, R. A. 1993, in Star-Forming Galaxies and their Interstellar Media, ed. J. J. Franco (Cambridge: Cambridge Univ. Press), 273

Shapiro, P. R., \& Field, G. B. 1976, ApJ, 205, 762

Shelton, R. 1998, ApJ, 504, 785

Shelton, R. L., Henley, D. B., \& Dixon, W. V. 2010, ApJ, 722, 302

Shull, J. M., \& van Steenberg, M. E. 1985, ApJ, 298, 268

Skillman, E. D. 1998, in VIII Canary Islands Winter School of Astrophysics: Stellar Astrophysics for the Local Group, ed. A. Aparicio, A. Herrero, \& F. Sánchez (New York: Cambridge Univ. Press), 457

Slavin, J. D., Shull, J. M., \& Begelman, M. C. 1993, ApJ, 407, 83

Spitzer, L. 1956, ApJ, 124, 20

Spitzer, L. 1990, ARA\&A, 28, 71

Spitzer, L. 1996, ApJ, 458, L29

Sterling, N. C., Savage, B. D., Richter, P. R., Fabian, D., \& Sembach, K. R. 2002, ApJ, 567, 354

Sutherland, R. S., \& Dopita, M. A. 1993, ApJS, 88, 253

Tripp, T. M., Sembach, K. R., \& Savage, B. D. 1993, ApJ, 415, 652

Wakker, B. P. 2001, ApJS, 146, 463 
Wakker, B. P. 2004, in High-velocity Clouds, ed. H. van Woerden, B. P. Wakker, U. J. Schwarz, \& K. S. de Boer (Astrophysics and Space Science Library, Vol. 312; Dordrecht: Kluwer), 25

Wakker, B. P. 2006, ApJS, 163, 282

Wakker, B. P., Howk, J. C., Savage, B. D., et al. 1999, Nature, 400, 388

Wakker, B. P., \& Savage, B. D. 2009, ApJS, 182, 378
Wakker, B. P., Savage, B. D., Sembach, K. R., et al. 2003, ApJS, 146, 1 Wakker, B. P., York, D. G., Howk, J. C., et al. 2007, ApJ, 670, L113

Welsh, B. Y., Edelstein, J., Korpela, E. J., et al. 2007, A\&A, 472, 509

Williamson, F. O., Sanders, W. T., Kraushaar, W. K., et al. 1974, ApJ, 193, L133

York, D. G. 1974, ApJ, 193, L127

York, D. G. 1977, ApJ, 213, 43

Zsargó, J., Sembach, K. R., Howk, J. C., \& Savage, B. D. 2003, ApJ, 586, 1019 